\title{
INJECTION-LOCK AND RECONFIGURABLE \\ CHARGE-DOMAIN SAMPLING MIXERS/FILTERS FOR DATA COMMUNICATIONS OVER WIRELESS CHANNELS
}

by

\author{
Yushi Zhou
}

Bachelor of Engineering, University of Electronic Science and Technology of China, 2000

\author{
A dissertation \\ presented to Ryerson University \\ in partial fulfillment of the \\ requirements for the degree of \\ Doctor of Philosophy \\ in the Program of \\ Electrical and Computer Engineering
}

Toronto, Ontario, Canada, 2015

(C) Yushi Zhou 2015 


\section{AUTHOR'S DECLARATION FOR ELECTRONIC SUBMISSION OF A DISSERTATION}

I hereby declare that I am the sole author of this dissertation. This is a true copy of the dissertation, including any required final revisions, as accepted by my examiners.

I authorize Ryerson University to lend this dissertation to other institutions or individuals for the purpose of scholarly research.

I further authorize Ryerson University to reproduce this dissertation by photocopying or by other means, in total or in part, at the request of other institutions or individuals for the purpose of scholarly research.

I understand that my dissertation may be made electronically available to the public. 


\begin{abstract}
Yushi Zhou

Injection-Lock and Reconfigurable Charge-Domain Sampling Mixers/Filters for Data Communications Over Wireless Channels

Doctor of Philosophy, Electrical and Computer Engineering, Ryerson University, 2015
\end{abstract}

This thesis provides a theoretical and experimental study of injection locking and reconfigurable charge-domain sampling mixers and filters for data communications over wireless channels.

On injection-locking, the intrinsic relation between the characteristics of injection signals such as sinusoidal or square, single-tone or multi-tone, the type of oscillators under injection such as harmonic oscillators (passive or active LC oscillators) or non-harmonic oscillators (ring or relaxation oscillators), and the lock range of the oscillators under injection was investigated. For the very first time, we discovered the intrinsic relation between the lock range and the phase of multiple injections of harmonic oscillators. In addition, we obtained the closed-form expression of the lock range of harmonic oscillators with square-wave injections. Moreover, we obtained the distinct characteristics of the lock range of harmonic and non-harmonic oscillators and that of different types of non-harmonic oscillators. These theoretical findings were not known before and were validated using simulation results.

On reconfigurable charge-domain sampling mixers and filters for software-defined radio, a novel quadrature charge-domain down-conversion sampling mixer with embedded finite-impulse-response (FIR), infinite-impulse-response (IIR), and 4-path bandpass filters was developed. An in-depth investigation of the principles of periodic impulse sampling, periodic windowed sampling, and periodic N-path windowed sampling was presented and a detailed mathematical treatment of charge-domain windowed samplers with built-in sinc, FIR and IIR filters was provided. The proposed quadrature charge-domain sampler with embedded FIR, IIR, and 4-path band-pass filters was implemented in IBM $130 \mathrm{~nm}$ 1.2V CMOS 
technology and its performance was validated both using simulation results and on-wafer measurement. 


\section{ACKNOWLEDGMENTS}

First and foremost, I would like to express my most sincere gratitude to my advisor, Professor Fei Yuan, for his kind guidance, helpful discussions and constructive suggestions throughout my study. I could not have completed this work without his support and enthusiasm.

I also thank the other committee members, Professor Andy Ye, Professor Vadim Geurkov, Professor GuangJun Liu and Professor Wai Tung Ng from University of Toronto for their invaluable feedback.

I wish to thank my fellow students in the Integrated Circuits and Systems Research Group, who were sitting around me and made the long journey of the study enjoyable. Special acknowledgements go to Young Jun Park, Alaa AL-Taee, Guangyu Zhu, Shaul Peker, Xiongliang Lai for their helpful discussions and suggestions for my research and career. I also would like to take the opportunity to thank the former members, Dr. Adrian Tang and Dr. Dominic DiClemente for their effort in establishing the test facilities, and Tarek Khan for creating the Cadence simulation environments. I am also indebted to staffs Jason Naughton, Dan Giannitelli and Bruce Derwin for their timely technical support.

Thanks to the Canadian Microelectronics Corporation (CMC) for making the fabrication of my chips. Also, I am grateful to the Natural Sciences and Engineering Research Council of Canada (NSERC), a significant funding source supporting my study (Canada Graduate Scholarships-CGS2) during 2013-2015.

Most importantly, I must thank my parents, brothers for providing me with much needed support and guidance over the years. Very special thanks to my wife, Zhaoying for her love and understanding throughout this endeavour. Finally, I wish to offer my gratitude to my lovely son, Alex, bringing joyfulness to me. 


\section{Table of Contents}

Table of Contents vi

List of Tables $\quad$ ix

List of Figures $\quad$ x

List of Symbols $\quad$ XV

1 INTRODUCTION 1

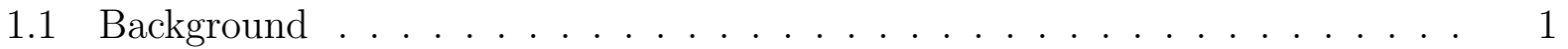

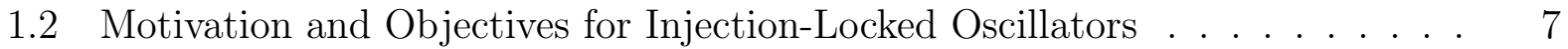

1.2.1 Injection-Locked Active Inductor Oscillators . . . . . . . . . . . . . 7

1.2.2 Injection-Locked Non-Harmonic Oscillators . . . . . . . . . . . . . . 8

1.3 Motivation and Objectives for Charge-Domain Sampling Circuits with Tunable Band-Pass Filter . . . . . . . . . . . . . . . . . . . . . 10

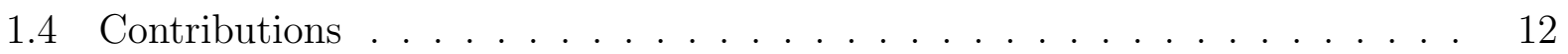

1.4.1 Injection-Locked Oscillators . . . . . . . . . . . . . . . 12

1.4.2 Tunable Band-Pass Filter Embedded Charge-Domain Sampling Circuits 12

1.5 Thesis Organization . . . . . . . . . . . . . . . . . . . . . 13

2 Injection-Locked Active Inductor Oscillators 15

2.1 Injection-Locked Oscillators . . . . . . . . . . . . . . . . 15

2.2 Active Inductor Oscillators . . . . . . . . . . . . . . . . . . 18 
2.3 Lock Range of Generic LC Oscillators . . . . . . . . . . . . . . . . . . 20

2.4 Lock Range and Tank Impedance Variation . . . . . . . . . . . . . . . 24

2.5 Comparison of Injection-Locked LC Oscillators and Active Inductor Oscillators 27

2.6 Summary . . . . . . . . . . . . . . . . . . . . . 32

$3 \quad$ Multiple Multi-Tone Injection-Locked Oscillators 34

3.1 Representation of Non-Harmonic Oscillators . . . . . . . . . . . . . . . 34

3.2 Representation of Injection-Locked Non-harmonic Oscillators . . . . . . . . . 36

3.2 .1 Single-Tone Injection . . . . . . . . . . . . . . . . . . 36

$3.2 .2 \quad$ Multi-Tone Injection $\ldots \ldots \ldots$

3.3 Harmonic Oscillators with Multiple Injections . . . . . . . . . . . . . . 39

3.4 Non-Harmonic Oscillators with Multiple Injections . . . . . . . . . . . . . . 41

3.5 Lock Range of Non-Harmonic Oscillators with Multiple Single-Tone Injections 42

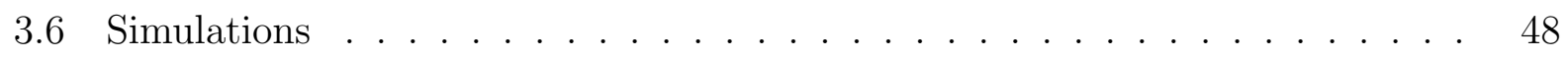

3.7 Lock Range of Non-Harmonic Oscillators with Multiple Multi-Tone Injections 49

3.8 Simulations $\ldots \ldots \ldots \ldots \ldots \ldots \ldots$

3.9 Summary . . . . . . . . . . . . . . . . . . . . 62

4 A Quadrature Charge-Domain Sampling Mixer with Embedded FIR, IIR, and N-Path Filters $\quad 64$

$4.1 \quad$ N-path Filters . . . . . . . . . . . . . . . . . . . . . . . . . 65

4.1 .1 Periodic Impulse Sampling . . . . . . . . . . . . . . . 65

4.1 .2 Periodic Windowed Sampling . . . . . . . . . . . . 66

4.1 .3 Periodic N-Path Windowed Sampling . . . . . . . . . . . . . 68

4.2 Charge-Domain Windowed Sampling . . . . . . . . . . . . . 73

4.2 .1 Sinc Low-Pass Filter . . . . . . . . . . . . . . . . . . 73 
$4.2 .2 \quad$ FIR Filter $\ldots \ldots \ldots \ldots \ldots \ldots$

$4.2 .3 \quad$ IIR Filter $\ldots \ldots \ldots \ldots \ldots \ldots$

4.3 Circuit Design and Analysis _ . . . . . . . . . . . . . . . 86

4.3 .1 Performance . . . . . . . . . . . . . . . . . . . 88

$4.3 .2 \quad$ Noise . . . . . . . . . . . . . . . . . . . . . . . . . . . . . . . . 90

4.3 .3 Effect of Nonidealities _ . . . . . . . . . . . . . . . . . . . . 94

4.4 Measurement Results . . . . . . . . . . . . . . . . . . . . . . . . . . . . 97

4.5 Summary . . . . . . . . . . . . . . . . . . . . . . 104

5 A Cascaded Charge-domain Sampling Mixer with Embedded $\operatorname{sinc}^{3}$ FIR $\begin{array}{lr}\text { and N-Path Filters } & 106\end{array}$

5.1 Hight-Order FIR Filter . . . . . . . . . . . . . . . . . . 106

5.2 Circuit Design and Analysis _ . . . . . . . . . . . . . . . . . . 108

5.2 .1 Performance . . . . . . . . . . . . . . . . . . . . . . 109

5.3 Simulation Results . . . . . . . . . . . . . . . . . . . . . . 111

5.4 Summary . . . . . . . . . . . . . . . . . . . . . . . . . . . 112

6 Conclusions and Future Work 115

6.1 Conclusions . . . . . . . . . . . . . . . . . . . . . . . 115

6.2 Future Work . . . . . . . . . . . . . . . . . . . . . . 116

$\begin{array}{ll}\text { Bibliography } & 118\end{array}$ 


\section{List of Tables}

3.1 Lock Range Coefficient $\alpha_{d} \ldots \ldots \ldots \ldots$. . . . . . . . . . . 47

4.1 Noise Bandwidth of Sampling Mixer with 4-path Bandpass, sinc, FIR, and IIR Filters Considered Individually. . . . . . . . . . . . . . . . . . . 93

4.2 Performance Summary of Sampling Mixer _ . . . . . . . . . . . . . 105

5.1 Performance Comparison . . . . . . . . . . . . . . . . . . . . . . . . . . 114 


\section{List of Figures}

1.1 Three receiver architectures: (a) Super-heterodyne receiver, (b) Zero-IF receiver, and (c) SDR receiver. . . . . . . . . . . . . . 5

1.2 Spectrum allocation for wireless communications. . . . . . . . . . . . . 7

2.1 A feedback system. . . . . . . . . . . . . . . . . . . 15

2.2 Injection locked procedure represented in simplified spectrum diagram. (a)Unlocked, (b) Fast beat, and (c) Locked. . . . . . . . . . . . . . . . . . 17

2.3 Single-ended active inductor. . . . . . . . . . . . . . . . . . . . 19

2.4 Examples of active inductors. (a) Wu current reuse active inductor , (b) Lin active inductor, and (c) Hara active inductor . . . . . . . . . . . . . . 20

2.5 (a) Injection-locked oscillators. (b) Block diagram of injection-locked oscillators. 21

2.6 Impedance of the LC tank of injection-locked LC oscillators. . . . . . . . . . 22

2.7 (a) Injection-locked oscillators. (b) Equivalent circuit of injection-locked oscillators. The dotted line represents the real injection signal, which has been merged into the variation of the LC tank. . . . . . . . . . . . . . . . . .

2.8 Injection-locked passive LC oscillator. $C_{1}$ and $C_{2}$ are accumulation MOS varactors. Circuit parameters : $W_{1,2}=10 \mu \mathrm{m} ; W_{3}=15 \mu \mathrm{m}, W_{4,5}=1 \mu \mathrm{m}$,

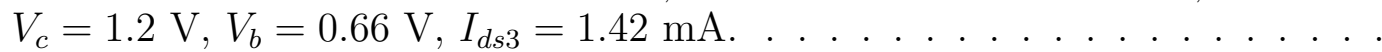

2.9 Schematic of injection-locked active-inductor VCO with $\mathrm{Wu}$ active inductors. Circuits parameters : $W_{1 a, 1 b}=40 \mu \mathrm{m}, W_{2 a, 2 b}=18.9 \mu \mathrm{m}, W_{3 a, 3 b}=4 \mu \mathrm{m}, W_{4,5}=$ $10 \mu \mathrm{m}, W_{6}=15 \mu \mathrm{m}, W_{7,8}=1 \mu \mathrm{m}, V_{b 1}=0.34 \mathrm{~V}, V_{b 2}=0.48 \mathrm{~V}, V_{b 3}=0.7 \mathrm{~V}$, $I_{d s 3}=131 \mu \mathrm{A}, I_{d s 6}=1.42 \mathrm{~mA} \ldots \ldots \ldots \ldots$

2.10 Simulated phase noise of passive LC oscillator $I_{i n j} / I_{o}=0.05 . \quad$. . . . . . 31

2.11 Simulated phase noise of active-inductor LC oscillator $I_{i n j} / I_{o}=0.05 . \quad$. . . 31

2.12 Simulated lock range. . . . . . . . . . . . . . . . . . . . . 32 
2.13 Asymmetry of the lock range of injection-locked active-inductor oscillator. .

3.1 Waveform and spectrum of non-harmonic oscillators. . . . . . . . . .

3.2 Representation of injection-locked non-harmonic oscillators with a single-tone injection. . . . . . . . . . . . . . . . . . 37

3.3 Injection-locked harmonic oscillators with multiple injections. . . . . . . . . . 40

3.4 Representation of injection-locked non-harmonic oscillators with multiple singletone injections. . . . . . . . . . . . . . . . . . . . . 42

3.5 Schematic of relaxation oscillator with injection-locking. Circuit parameters: $W_{1,2,6,7,10,11}=0.5 \mu \mathrm{m}, W_{3,4}=1.2 \mu \mathrm{m}, W_{8,9,12,13}=1.25 \mu \mathrm{m}, W_{5}=2 \mu \mathrm{m}$, Supply voltage $V_{D D}=0.9 \mathrm{~V}, V_{\text {ref }}=0.31 \mathrm{~V}, I_{b}=253 \mathrm{nA}, V_{b}=0.62 \mathrm{~V} \ldots \ldots 48$

3.6 Simulated spectrum of relaxation oscillator without injection. Top - Fundamental. Middle : 3rd harmonic. Bottom : 5th harmonic. . . . . . . . . . . 50

3.7 Simulated spectrum of relaxation oscillator in fast beat. Top - Fundamental. Middle : 3rd harmonic. Bottom : 5th harmonic. . . . . . . . . . . 51

3.8 Simulated spectrum of relaxation oscillator in quasi-lock. Top - Fundamental. Middle : 3rd harmonic. Bottom : 5th harmonic. . . . . . . . . . . . 52

3.9 Simulated spectrum of relaxation oscillator in lock state. Top - Fundamental. Middle : 3rd harmonic. Bottom : 5th harmonic. . . . . . . . . . . 53

3.10 Simulated dependence of the lock range of relaxation oscillator with a sinusoidal injection and double sinusoidal injections whose phase difference is 180 degree on injection strength. . . . . . . . . . . . . . .

3.11 Representation of injection-locked non-harmonic oscillators with multiple multitone injections. . . . . . . . . . . . . . . . .

3.12 Simulated dependence of the lock range of relaxation oscillator with a single square-wave injection and with double square-wave injections whose phase difference is 180 degree on injection strength. . . . . . . . . . . . . . .

3.13 Simulated dependence of the lock range of relaxation oscillator with differential square-wave injection on injection strength. . . . . . . . . . . .

3.14 Simulated dependence of the lock range of relaxation oscillator with a single sinusoidal injection, a single square-wave injection, and a single dual-tone (two-sinusoids) injection on injection strength. . . . . . . . . . . . 
3.15 Simulated dependence of the lock range of relaxation oscillator with double square-wave injections on the phase difference of the injection signals. The

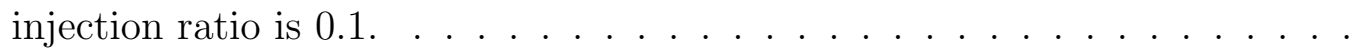

3.16 Simulated dependence of the lock range of relaxation oscillator with a two-tone sinusoidal injection and double two-tone injections on injection strength. .

3.17 Simulated dependence of the lock range of relaxation oscillator with double single-tone injections, double two-tone injections, and double square-wave injections on injection strength. . . . . . . . . . . . . .

4.1 Periodic impulse sampling of band-limited signals. . . . . . . . . . . . . .

4.2 (a) Periodic impulse sampling of band-limited signals. Baseband signal at $\omega$ is replicated to sidebands at $\omega+n \omega_{s}$. (b) Periodic impulse sampling of broadband signals. The sideband components of input at $\omega+n \omega_{s}$ are aliased

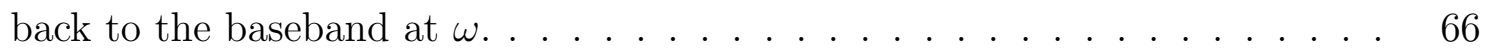

4.3 Periodic windowed sampling of band-limited signals. . . . . . . . . . . . 67

4.4 (a) Periodic windowed sampling of narrow-band signals without interferences. (b) Periodic windowed sampling of band-limited signals with interferences. Interferences at $\omega+n \omega_{s}$ are aliased back to the desired baseband. . . . . . .

4.5 N-path filter. Sampling mixers are implemented using switches drived by a sampling clock with $N$ non-overlapping phases. Non-overlapping uniform sampling pulse width: $\tau=T_{s} / N . \sigma_{k}=\sigma_{k-1}+\tau$ and $\sigma_{k-1}=n T_{s}+(k-1) \tau$. .

4.6 Single-ended 4 -path filter. . . . . . . . . . . . . . . . . 72

4.7 Single-ended 4 -path filter. . . . . . . . . . . . . . . . . 73

4.8 Frequency response of single-ended 4-path filter in Fig. 4.6. . . . . . . . . . . 74

4.9 Charge-domain sampling. Solid line : Small $T_{i}$. Dashed line : Large $T_{i} . \ldots 74$

4.10 Gain-normalized transfer function of sinc. . . . . . . . . . . . . 75

4.11 Time interleaved charge-domain sampling. . . . . . . . . . . . . . . 77

4.12 Charge-domain sampler with built-in FIR filter. . . . . . . . . . . . 78

4.13 Spectrum of charge-domain windowed sampler with FIR filtering. Parameters: $f_{s}=10 \mathrm{MHz}, N=10$ and $T_{f}=T_{s} / N \ldots \ldots \ldots \ldots$

4.14 Spectrum of charge-domain windowed sampler with FIR filtering. Parameters: $f_{s}=20 \mathrm{MHz}, N=5$ and $T_{f}=T_{s} / N$. 
4.15 Spectrum of charge-domain windowed sampler with CT sinc low-pass filter. $f_{i}=200 \mathrm{MHz}, f_{s}=10 \mathrm{MHz}, N=10$ and $T_{f}=T_{s} / N \ldots \ldots \ldots$

4.16 Charge-domain sampler with a build-in FIR and IIR filter and its timing scheme.

4.17 Time interleaved charge-domain sampler with a build-in FIR and IIR filter and its timing scheme. . . . . . . . . . . . . . . . . .

4.18 Spectrum of charge-domain windowed sampler with first-order IIR filter. $f_{s}=10$ $\mathrm{MHz}$.

4.19 Quadrature Charge-Domain Sampler with 4-path bandpass filter. Circuit Parameters: $W_{1,2}=20 \mu \mathrm{m}, W_{3,4,5}=15 \mu \mathrm{m}, W_{6,7}=10 \mu \mathrm{m}, L=0.36 \mu \mathrm{m}$, $I_{b}=150 \mu \mathrm{A} ., V_{b 1}=0.67 \mathrm{~V}, V_{b 2}=0.65 \mathrm{~V} \ldots \ldots \ldots \ldots \ldots$

4.20 (a) Differential 4-path band-pass filter, (b) Clocking scheme. . . . . . . . . . 87

4.21 Simulated differential 4-path band-pass filter with $T_{s}=T_{L O}=100 \mathrm{MHz} . \quad$. 87

4.22 (a) Differential charge-domain sampler (only half of the circuit is shown), (b) Clocking scheme.

4.23 Simulated transfer function (normalized) of quadrature charge-domain sampler. Ideal transconductor with $g_{m}=0.5 \mathrm{mS}$ and ideal switches with $R_{o n}=$ $1 \Omega$ and $R_{\text {off }}=1 \mathrm{~T} \Omega$ are used. The input is a $100 \mathrm{MHz}$ sinusoid. The sampling frequency is $100 \mathrm{MHz}$ with $25 \%$ duty cycle. Voltage gain provided by

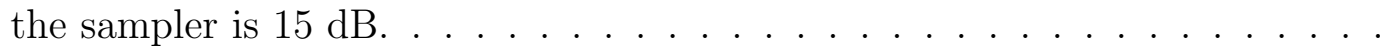

4.24 Noise analysis of proposed charge-domain sampling mixer. . . . . . . . . . . 90

4.25 Noise analysis of N-path filter. . . . . . . . . . . . . . . . . . . . . . . 92

4.26 Simulated effect of ON-resistance of switches on 4-path bandpass filter. $R_{o n}$ is varied from $5 \Omega$ to $200 \Omega$. . . . . . . . . . . . . . . . . . .

4.27 Simulated frequency response (normalized) of quadrature charge-domain sampler with embedded 4-path bandpass filter at process corners. The input frequency is $100 \mathrm{MHz}$, the output baseband sampling frequency is $25 \mathrm{MHz}$ (Two $12.5 \mathrm{MHz}$ interleaved clocks with $50 \%$ duty-cycle). Legends: TT (typical nMOS/typical pMOS), SS (slow nMOS/slow pMOS), FS (fast nMOS/slow pMOS), SF (slow nMOS/fast pMOS), and FF (fast nMOS/fast pMOS). . . .

4.28 Simulated frequency tuning range. The input frequency is varied from $50 \mathrm{MHz}$ to $250 \mathrm{MHz}$. The sampling frequency is also varied accordingly such that the output frequency remains unchanged. 
4.29 Die microphotograph of quadrature charge-domain sampler $\left(1 \mathrm{x} 2 \mathrm{~mm}^{2}\right) . \quad$. . 98

4.30 (a) Active balun. (b) Output buffer. Circuit parameters of active balun: $W_{1,2}=300 \mu \mathrm{m}, R_{s 1}, R_{D 1}, R_{D 2}=200 \Omega$. Circuit parameters of source follower : $W=300 \mu \mathrm{m} . R_{s}=1 \mathrm{k} \Omega$. Length of all transistors is $0.13 \mu \mathrm{m} . . . . . . .$.

4.31 Die photo-micrograph of charge-domain sampler with embedded 4-path bandpass filter with probes in place for on-wafer measurement. . . . . . . . . . . 99

4.32 Measurement set-up. . . . . . . . . . . . . . . . . . . 100

4.33 Measured quadrature output voltage of mixing sampler with input at 100.1 MHz. . . . . . . . . . . . . . . . . . . . . 102

4.34 Measured and simulated normalized transfer function of mixing sampler. . . 102

4.35 Measured tuning range of mixing sampler. . . . . . . . . . . . . . . 103

4.36 Post-layout simulated IIP3. . . . . . . . . . . . . . . . 103

4.37 Simulated $P_{1 d B} \ldots \ldots \ldots \ldots$

4.38 Post-layout simulated IMR. . . . . . . . . . . . . . . . . . . . 104

5.1 Frequency response of sinc, $\operatorname{sinc}^{2}$ and $\operatorname{sinc}^{3} \cdot f_{s, f i r}=10 \mathrm{MHz}$, decimation ratio

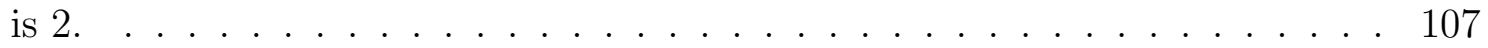

5.2 Advanced charge-domain sampler with embedded N-path band-pass filter.Circuit Parameters: $g m_{1}=2 \mathrm{mS}, g m_{4}=1.5 \mathrm{mS}, g m_{6}=4.5 \mathrm{mS}, I_{b 1}=150 \mu \mathrm{A}, I_{b 3}=$ $235 \mu \mathrm{A}, I_{b 5}=700 \mu \mathrm{A}, V_{b}=335 \mathrm{mV} \ldots \ldots \ldots \ldots$

5.3 Response of 4-path band-pass filter in Fig. 5.2 ( $v_{o 1}$ excluding M3-M6 and CDS).109

5.4 High-order windowed charge-domain sampler. Circuit Parameters: $g_{m}=1.5$ $\mathrm{mS}, C_{S 1}=10 \mathrm{pF}, C_{S 2}=1 \mathrm{pF} \ldots \ldots \ldots \ldots \ldots$

5.5 Simulated gain-normalized transfer function of proposed charge-domain windowed sampler with $100 \mathrm{MHz}$ input. . . . . . . . . . . . . . 111

5.6 Simulated time response of the output signal with $V_{\text {in }}=50 \mathrm{mV}, f_{\text {in }}=100.2$ $\mathrm{MHz}, V_{\text {out }}=170 \mathrm{mV}, f_{\text {out }}=200 \mathrm{KHz}, f_{\text {s,out }}=25 \mathrm{MHz} \ldots \ldots \ldots$. . . . 112

5.7 Simulated time response of the output signal with $V_{\text {in }}=50 \mathrm{mV}, f_{\text {in }}=100.2$ $\mathrm{MHz}, V_{\text {out }}=170 \mathrm{mV}, f_{\text {out }}=200 \mathrm{KHz}, f_{\text {s,out }}=25 \mathrm{MHz} \ldots \ldots \ldots$. . . . 113

5.8 Post-simulated time response of the output signal with $V_{\text {in }}=40 \mathrm{mV}, f_{\text {in }}=$ $100.2 \mathrm{MHz}, V_{\text {out }}=140 \mathrm{mV}, f_{\text {out }}=200 \mathrm{KHz}, f_{\text {s,out }}=25 \mathrm{MHz}$. . . . . . . . 113 


\section{List of Symbols}

$\begin{array}{ll}A D S & \text { Advanced Design Systems } \\ A I & \text { Active Inductor } \\ A M & \text { Amplitude Modulation } \\ B P & \text { Band-Pass } \\ C A D & \text { Computer-Aided Design } \\ C D S & \text { Charge-Domain-Sampler } \\ C H F & \text { Channel Selection Filter } \\ C M O S & \text { Complementary Metal-Oxide Semiconductor } \\ d B m & \text { Decibel power with respect to 1 Milliwatt } \\ E M & \text { Electromagnetic } \\ E P C & \text { Gen2 Electronic Product Code Generation } 2 \\ F F & \text { (nMOS)Fast-(pMOS)Fast } \\ F M & \text { Frequency Modulation } \\ F S & \text { (nMOS)Fast-(pMOS)Slow } \\ F S K & \text { Frequency-Shift Keying } \\ I M F & \text { Image Rejection Filter } \\ I L A I O & \text { Injection-Locked Active Inductor Oscillator } \\ I L O & \text { Injection-Locked Oscillator } \\ \text { F } & \end{array}$




\begin{tabular}{|c|c|}
\hline$I L F D$ & Injection-Locked Frequency Divider \\
\hline$I S M$ & Industrial Scientific Medical (radio bands) \\
\hline ISO & International Organization for Standardization \\
\hline$L C$ & Inductor-Capacitor \\
\hline$L N A$ & Low Noise Amplifier \\
\hline$L O$ & Local Oscillator \\
\hline$L P$ & Low-Pass \\
\hline$M I M(-$ сар $)$ & Metal-Insulator-Metal (Capacitor) \\
\hline$n M O S$ & n-channel Metal-Oxide Semiconductor \\
\hline$P L L$ & Phase-Locked Loop \\
\hline$p M O S$ & p-channel Metal-Oxide Semiconductor \\
\hline POR & Power-ON Reset \\
\hline$P V T$ & Process (supply-)Voltage Temperature \\
\hline$R F$ & Radio Frequency \\
\hline$R F I D$ & Radio Frequency Identification system \\
\hline$R L C$ & Resistor-Inductor-Capacitor \\
\hline$S A W$ & Surface Acoustic Wave \\
\hline$S D R$ & Software Defined Radio \\
\hline$S F$ & (nMOS)Slow-(pMOS)Fast \\
\hline$S S$ & (nMOS)Slow-(pMOS)Slow \\
\hline$T T$ & (nMOS)Typical-(pMOS)Typical \\
\hline$U H F$ & Ultra High Frequency \\
\hline $\mathrm{VCO}$ & Voltage-Controlled Oscillator \\
\hline
\end{tabular}




\section{Chapter 1}

\section{INTRODUCTION}

\section{$1.1 \quad$ Background}

Communication systems are the foundation of information transfer. Based on the transmission media, communication systems are generally categorized into wireless and wireline communications. Wireline communications is often referring to high speed signaling through cables, or micro-strip, which is used in a wide variety of digital systems, i.e. the computer, digital television, sensor networks and data storage center, to name a few. It is now the dominant method for long distance telecommunications and extremely high speed data transmission in short range, e.g. field programmable gate arrays (FPGA) to memory, graphics processing unit (GPU) to memory and Gigabit Ethernet. Oscillators are widely used as local clock generators in phase-lock loop and clock distributions network. Unlike wireline communications, wireless communications is usually making use of radio to transfer information between two or more points which are not physically connected via any electrical conductors. The dramatically increased demand from cell phones and portable mobile products is a drive force to make wireless communications to be the fastest growing segment of the communications industry in the past two decades [1]. The design of low-power, low-cost and high performance radio frequency $(\mathrm{RF})$ transceivers becomes a challenge. Most of the transceivers extensively use filters and oscillators as local oscillators, RF filters, intermediate frequency (IF) filters and channel selection filters, which cover the frequencies from $200 \mathrm{KHz}$ to multi-GHz, depending on their locations in the transceivers [2]. 
One of the key components in transceivers is the frequency synthesizer, synthesizing various RF frequency according to the architecture of transceivers and communication standards. In the integer- $\mathrm{N}$ frequency synthesizer, the pre-scalar is the first stage to lower the frequency by integer division. In general, pre-scalars are implemented in a digital form by two static latches connected in a negative feedback loop. It can also be implemented by dynamic latches, e.g. true single-phase clock (TSPC) based divider. The popularity of these two types of digital dividers in RF transceivers is because it is widely believed that the simple structure, the larger bandwidth, and the better robustness of digital dividers over process variations outperform their analog counterparts, e.g. Miller frequency divider $[3,4]$ and parametric frequency divider [5]. For both static and dynamic pre-scalars, however, a trade-off between the speed and power consumption becomes more critical as the speed increases. The solution to balance the performance and power dissipation is to use injection-locked oscillators to substitute the conventional digital divider.

When an incident signal is applied to the oscillator, it is changed from a free-running oscillator to a forced oscillator. The free-running frequency shifts towards the frequency of the incident signal under a certain condition. The detailed description will be given in Chapter 2.1. A simple injection-locked frequency divider (ILFD), so called super-harmonic injection-locked oscillator, is an negative resistor pair LC tank oscillator. The incident signal with higher frequency is injected into the tail transistor. The output of the differential pair shows that the frequency is divided by 2 , which fulfils the function of pre-scalar. This structure was analyzed in terms of phase limited and amplitude limited locking range and the noise characteristics in [6]. It achieved locking range by $12.3 \%$ at $3 \mathrm{GHz}$, and later it was used as a pre-scalar to construct a PLL in [7]. Instead of injecting the signal to the tail transistor, the injection signal can be directly injected into the LC tank. [8] proposed a low power quadrature generation circuit by injecting signals to two identical ILFDs. A hard switching model was conceived to give rise to simple expressions for the lock range and output amplitude. [9] presented a wider lock-range high-speed pre-scalar at $50 \mathrm{GHz}$ with a combination of injection-locked oscillators. Except for divide-by-2, odd number superharmonic ILFD was reported [10, 11, 12, 13, 14, 15]. In [14] and [15], the author presented a divided-by-3 pre-scalar, running at higher than $100 \mathrm{GHz}$ frequency. For these designs, 
one extra inductor or even more inductors were required to create high impedance at $3 \omega_{o}$ ( $\omega_{o}$ is the fundamental frequency). A current-mode logic frequency divider was described in [16], which showed $4.1 \mathrm{GHz}$ lock range at $24 \mathrm{GHz}$ frequency. The divide-by-4 was obtained through two divider stages with $0.8 \mathrm{~V}$ power supply. It overcame the constrain that the low power supply limits the highest achievable frequency of current mode logic. Apart from injection-locked frequency divider, quadrature injection-locked oscillators are exceptional candidates that can be employed in the low-power high-performance quadrature clock generators $[17,18,19,20,21,22]$. Injection-locked oscillators can also be found in the wireline communications, e.g. reducing timing jitter and clock deskew [23, 24, 25, 26, 27, 28]. Among these applications, the majority is the LC tank based oscillators due to their better noise performance and lower power dissipation. Moreover, there are some other kinds of oscillators for different applications, such as ring oscillators and relaxation oscillators.

Owing to their sensitivity to temperature variation and supply voltage fluctuation, non-harmonic oscillators such as relaxation oscillators are widely used in passive wireless microsystems (PWMs) such as implanted medical devices, embedded sensors, and radiofrequency identification tags to generate system clocks that control both the operation of PWMs and their communications with base stations. A stringent frequency requirement for the system clock exists. For example, EPC radio-frequency identity protocols class-1 generation-2 UHF RFID protocols require that the accuracy of the frequency of the backscattered data be bounded by $\pm 4 \%$ [29]. Arising from the effect of process spread, supply voltage fluctuation, and temperature variation (PVT), the frequency of these oscillators exhibits a large degree of uncertainty. For example, the variation of the frequency of a free-running oscillator can be as large as $\pm 20 \%$ [30]. The uncertainty of the frequency of the system clock of PWMs is further escalated due to the fact that these systems are usually fabricated using low-cost CMOS technologies, which typically have a high degree of process spread. Calibrating the system clock of PWMs prior to their operation is required to ensure the proper operation of PWMs. The effect of supply voltage fluctuation and temperature variation can be compensated using bang-gap circuits [31, 32], their effectiveness is largely hindered by the limited power resources of PWMs, which severely limits the degree of the complexity of compensation circuitry subsequently their performance. Although a number of techniques 
such as digital trimming [33] and phase-locked loops [34] were used to perform the remote calibration of system clocks, both suffer from a long calibration time and high power consumption. Injection-locking that offers the intrinsic advantage of fast-locking, high frequency accuracy, small fluctuation in power flow, and improved phase noise performance emerged as a power-efficient method to calibrate the system clock of PWMs. Both the carrier [35] and envelope [36] of the signal from the base station were used as injection-locking signals. Injection-locking was also used in calibration of power oscillators for inductive power links [37], the fine frequency tuning of quadrature oscillators [38], self-cascade body-coupled oscillators for biomedical temeletry [39], wake-up receivers for wireless body area networks [40], and FSK transceivers for body sensor networks [41], to name a few. Because the maximum emission power from the base station is regulated, for example, in both EPC and ISO 18000-4, it is 4 watts EIRP (Equivalent Isotropically Radiated Power) and RF power drops quadratically with the distance between transmitters and receivers, the voltage generated by the antenna of PWMs for injection-locking is rather low. As a result, the success of injection-lock based remote frequency calibration is critically determined by the lock range of oscillators. Oscillators with a large lock range are highly desirable.

The other part that determines the performance of the transceivers is the architecture of the RF transceivers. Fig. 1.1 shows three possible architectures of the receivers. The most widely used architecture in RF world that has been for many decades is the super-heterodyne receiver, invented by Armstrong in 1917 [42]. It needs two analog mixers to complete two stages frequency translation, in which, at the first stage RF is translated to IF, and then in the second stage it is from IF to baseband signal. The diagram of this receiver is illustrated in Fig. 1.1(a).

In the super-heterodyne receiver, the incoming signal is selected by the high-Q bandselected filter and then amplified by the low-noise amplifier (LNA). To reject the strong signals in order to avoid blocking of the receiver, such a filter must be designed to have very high Q-factor, e.g. Q is larger than 100 for digital TV broadcasting. The discrete components, Surface Acoustic Wave (SAW) filters, are more common to be employed to meet the stringent requirements. An image rejection filter (IMF) is indispensable to be 


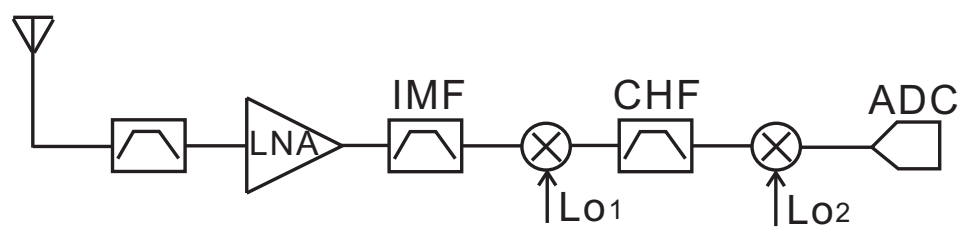

(a)

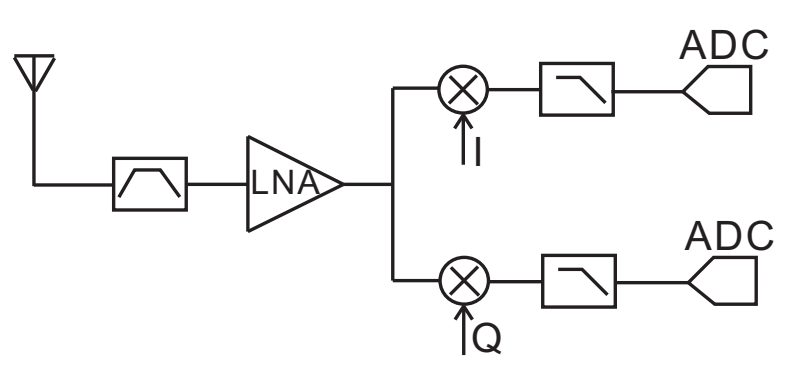

(b)

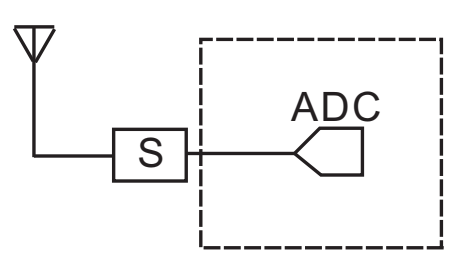

(c)

Figure 1.1: Three receiver architectures: (a) Super-heterodyne receiver, (b) Zero-IF receiver, and (c) SDR receiver.

inserted between LNA and Mixer to filter out the image signal. The signal at the output of the mixer is translated to a lower frequency, where analog or digital processing is easier to perform. A channel selection filter (CHF) performs channel selection at the IF. The last stage is to ensure that the desired signal is in baseband following by an analog-to-digital converter (ADC) to eventually quantize analog signal $[43,44,45,46]$. Although the super-heterodyne receivers are quite successful because of their simple structure and high selectivity, they suffer from several drawbacks, e.g. less attractive for highly integrated receiver because of external filters, extra rejections for image signals and impedance matching network in the intermediate stage requiring more design effort.

To eliminate the extra image rejection filters, zero-IF receivers is then proposed in Fig. 1.1(b). The key concept of this type receiver is to make LO's frequency same as $\mathrm{RF}$ signal, converting modulated RF signal to DC directly, thereby resolving image signal 
problem. The selected RF signal is multiplied by I/Q clock, generated from quadrature LO, to allow the receiver to choose high-side or low-side injection [47, 48, 49]. In [49], an ultra wide band receiver to cover frequency, 3 - $10 \mathrm{GHz}$, was presented. A LNA with embedded notch filter, providing average $-25 \mathrm{~dB}$ gain over wide frequency band, with tuning capability to attenuate other interferences was introduced. One step frequency translation and unnecessary image rejection filters are the major benefits of zero-IF receivers. However, the unavoidable drawbacks, e.g. DC offset errors because of LO-self mixing, LO leakage due to parasitic paths, intrinsic flicker noise of MOSFET transistors adding on top of the desired signal and I/Q mismatch $[2,50]$, existing in this architecture are still concerns.

The rapid advance of CMOS technologies has reached the level that analog RF systems can be implemented in such a way that sampled-data and digital signal processing techniques that traditionally championed in low-frequency applications can be utilized in design of RF front-end to reduce cost, improve performance, and enhance reconfigurability. Therefore, the optimal receiver with maximum flexibility and lowest cost for a receiver can be realized by pushing ADC towards antenna, Fig. 1.1(c). The RF signal will be directly sampled and quantized through ADC, introducing baseband digital signal processing into $\mathrm{RF}$. The ADC must be very linear and is capable to deal with large variation of signal range. Unlike analog mixers, the frequency translation can be done in digital domain, minimizing phase and gain error over wideband, which is the inherent obstacle in analog mixers. Such a concept, defined as software-defined radio (SDR) and was first proposed by Mitola in 1995 [51], and become more important recently. The importance of this architecture also stems from more complex spectrum planning, which was specified by different organizations (ITU, IEEE, 3GPP and etc.). Fig. 1.2 shows a couple of examples in the spectrum of $50 \mathrm{MHz}$ to $4 \mathrm{GHz}$, including short-range communication standards RFID, mobile digital TV standard DVB-T, cellular standards GSM, UMTS, and LTE, and the wireless networking standards Wi-Fi and WiMAX.

However, design such an ADC, meeting all the requirements, become more challenge. For example, ADC, applying to SDR receiver directly, must have sufficient dynamic range and handle both low and high power signals without any high-Q filtering [52]. As a result, 


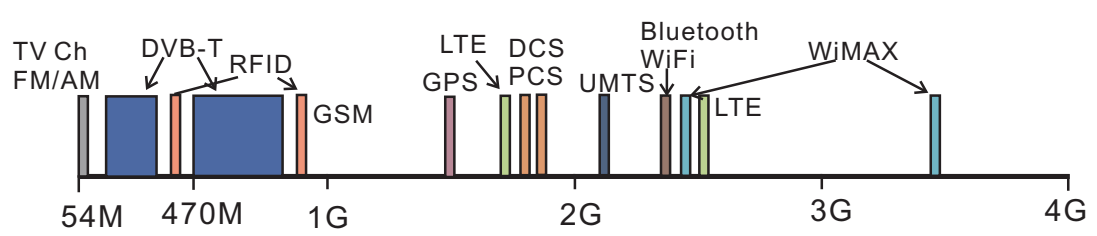

Figure 1.2: Spectrum allocation for wireless communications.

unacceptable power dissipation, usually in Watts, overwhelms benefits of the architecture. In comparison with pushing ADC towards antenna, as transceivers can be loosely partitioned into RF analog front-end and digital back-end with the digital back-end fully programmable, to have reconfigurable $\mathrm{RF}$ analog front-ends is more attractive.

\subsection{Motivation and Objectives for Injection-Locked Os- cillators}

\subsubsection{Injection-Locked Active Inductor Oscillators}

The oscillators that are employed in data communications over wireless systems are typically LC oscillators with spiral inductors or transformers, owing to the stringent phase noise requirements. On the other hand, LC oscillators suffer from small lock range without paralleling a number of varactors due to the passive components, e.g. passive inductors and capacitors, which also lead to large silicon consumption. One way to combat these problems is to use active devices, which provide a large tuning range of impedance, to substitute the passive devices, especially the large inductors are replaced with active inductors (AI), and varators are removed thereby saving a large amount of silicon area. Active-inductor-based oscillators, however, exhibit poor noise performance attributed to the more noisy devices being used. [53] showed that phase noise of an injection-locked oscillator can be reduced to the level comparable to that of the injecting signal in the lock state. The ability to lower the phase noise of injection-locked active inductor oscillators (ILAIO) to the level of the external "clean" signal and the large frequency tuning range make active inductor oscillators particularly attractive for injection-locked frequency dividers [54, 55, 13, 56, 57, 58]. Also 
the relative poor phase noise of injection-locked active inductor oscillators is not of a critical concern for some applications, e.g. phase shifters [59] and limiting amplifiers [60].

The analytical study of phenomenon of injection locked oscillators was first presented by Adler [61]. Later Razavi derived the well-known equations of lock range for CMOS oscillators in [53] by making use of a phasor domain approach. Recent published papers $[62,63,64]$, using different approaches to explain the locking process, continue to disclose new findings. None of them, however, can be applied to the injection-locked active inductor oscillators thereby deeply exploring the lock range. The mechanism of why ILAIOs provide large lock range is still unclear although it has been reported in many publications. Therefore, there is no doubt that the need to explore the relation between the lock range and the active inductor oscillator is of interest.

In this dissertation these issues will be resolved by exploiting 1) the intrinsic relation between the lock range and the injection-induced variation of the impedance of LC tank, 2) the lock range of injection-locked active inductor oscillators attributed to both the low quality factor and the large injection-induced variation of impedance. In addition, a simple but effective approach to derive the expression of the lock range will be presented.

\subsubsection{Injection-Locked Non-Harmonic Oscillators}

The preceding introduction for different applications of injection-locked oscillators will eventually come to the most widely studied subject: How to increase the lock range of injection-locked oscillators. A number of novel design techniques emerged to increase the lock range of injection-locked LC frequency dividers. Chang et al. showed that the lock range can be increased if the dc component of the voltage of the output of the oscillator is used to tune the varactor of the oscillator [65]. This is essentially a varactor-tracking technique that automatically forces the varactor voltage to track the control voltage of the oscillator so as to achieve an effective large lock range even though the absolute lock range of the oscillator is not increased. Since the lock range of injection-locked LC oscillators is inversely proportional to the quality factor of the oscillators, lowering the quality factor of the oscillators will effectively increase the lock range. Both series resistance [66] and shunt 
resistance [67] methods emerged to achieve this. To increase the lock range, the strength of internal locking signals that actually mixes with the output of the LC tank and determines the lock range must be maximized. Wu and Hajimiri showed that although the strength of an external injection signal is high, its strength is severely attenuated by the parasitic capacitances of the path over which the actual locking signal is generated. As a result, only a small lock range can be achieved in spite of a strong external injection signal [68]. To combat this, the internal locking signal must be maximized prior to mixing. Inductive series-peaking [69], sub-threshold mixing [10], and transformer positive feedback [70] have been proposed for this purpose. To determine the lock range of ring oscillators, a time-domain approach was given in [71]. Although a ring oscillator can lock to a locking signal injected to only one node of the oscillator, a larger lock range can be obtained if the locking signal is injected into the multiple stages of the oscillator simultaneously $[72,73]$. The lock range can be further increased if the phase of each of the multiple injections is properly chosen [69, 74]. Jin et al. showed that the lock range of injection-locked ring oscillators can be improved by employing a feedback resistor between the input and output of the oscillators [75]. The inserted resistance effectively lowers the quality factor of the oscillators so as to boost the lock range. Injection-locked relaxation oscillators were also studied in [76, 77, 78]. Soltani and Yuan showed that integrating feedback that integrates the difference between the frequency of the injection-locking signal and that of relaxation oscillators improves lock range [36].

The preceding narrow-band techniques for lock range enhancement of LC oscillators are not applicable to non-harmonic oscillators due to the fact that an infinite number of frequency components exist at the output of non-harmonic oscillators whereas only a singlefrequency component exists at the output of LC oscillators. The latter permits the use of impedance peaking techniques at a selected frequency and location to maximize the effective internal locking signal so as to achieve a large lock range. Although ring oscillators in several designs have been demonstrated to be able to yield a larger lock range, an in-depth study of the mechanism and factors that determine the lock range of non-harmonic oscillators and methods that increase the lock range of these oscillators are critically needed but not available yet. 
In this dissertation, the missing points will be added in the following chapters, including 1) an analytical treatment of the intrinsic relation between the phases of the multiple injection signals, which is essential in design of non-harmonic oscillators with multiple injections in order to yield a large lock range, 2) the intrinsic relation between the lock range of non-harmonic oscillators with single injection and that with multiple injections, though fundamentally important in understanding why non-harmonic oscillators with multiple injections can yield a large lock range, and 3) a comprehensive study of lock range for single-tone and multi-tone injections for non-harmonic oscillators, which was not appeared in any publications.

\subsection{Motivation and Objectives for Charge-Domain Sam- pling Circuits with Tunable Band-Pass Filter}

To handle different requirements for signal bandwidths and data rates, the most of commercial transceivers are often realized either by integrating multiple silicon component cores optimized for individual wireless standard on the same substrate or be packaging multiple silicon chip sets. Both approaches are costly. In addition, they provide inflexibility in coping with new standards in wireless communications. To overcome it, a number of design techniques have emerged for silicon implementation of SDR. Among them, charge-domain windowed sampling that provides an inherent sinc low-pass characteristic with FIR filtering to attenuate unwanted channels, suppresses the fold-over of broadband noise to the desired baseband, and nulling out aliasing interferences while exhibiting a low sensitivity to clock

jitter and consuming less power is a promising technique [79]. Muhammad and Staszewski showed that IIR filtering from the recursive operation of a history capacitor(charge residual) and rotating capacitors(charge shared) provide an additional $20 \mathrm{~dB}$ stop-band attenuation [80, 81]. However, it can only be applied to the bluetooth frequency band. By changing the sampling frequency, the ratio of the capacitance of the history capacitor to that of the rotating capacitors, and the number of the rotating capacitors, the characteristics of built-in low-pass and anti-aliasing filter can be made programmable [82, 83]. Unfortunately, these 
designs are less attractive because of the necessary of the external pre-selection for the suppression of out-of-band interferences. The active integrators employed in the designs also degrade the noise figure and linearity. Because a channel typically occupies a frequency band rather than a single frequency, the suppression of unwanted tones provided by these filters in the neighbor of the nulls is often insufficient $[84,85]$. Increasing the sampling frequency, though improving the suppression of unwanted tones in the vicinity of the nulls to a certain extent, increases power consumption. Mrizaei et al. showed that anti-aliasing and low-pass filtering from charge-domain windowed sampling can be improved simultaneously by employing $\operatorname{sinc}^{2}$ filtering $[52,74,86]$. Yoshizawa and Iida proposed a wide-band chargedomain baseband filter [87]. However, fixed-centre-frequency band-pass filter is still required in those receivers, making charge-domain sampling circuits less attractive for the increasing complexity of frequency bands. Recently, N-path filters widely popular in switched-capacitor band-pass filters have attracted a great interest to realize programmable high-Q band-pass filters $[88,89,90]$. The tunable center frequency and bandwidth, low cost, and full compatibility with digital CMOS make these filters particularly attractive for SDR. The common issue for the presented design is the lack of sufficient attenuation for the undesired signal accompanying with wanted signal deteriorates signal-to-noise ratio(SNR). In particular if the aliasing signal is on top of the desired signal, there is no doubt that the sensitivity of the receiver is lowered. The worst case is a large out of band or in band interference will block the receiver, severely degrading the performance of the overall RF communication system.

In this dissertation, the lack of filtering functions will be tackled by proposing new architectures, including 1) a new charge-domain quadrature downconversion circuit with a tunable band pass filter, 2) a new charge-domain sampling circuit with high-order FIR filter embedded. The sufficient study of principles of periodic impulse sampling, periodic windowed sampling, and periodic N-path windowed sampling will also be provided. Detailed mathematical treatments of charge-domain windowed samplers will be given. 


\subsection{Contributions}

The original contributions of the dissertation are summarized in this section.

\subsubsection{Injection-Locked Oscillators}

In this dissertation, the indispensable mathematical treatments for enlarging the lockrange of the ILAIOs are given, exploring the mechanism of how ILAOs are able to offer large lock range thereby providing a clear guidance of deigning ILAIOs. In addition, a systematic and in-depth investigation for the injection-locked non-harmonic oscillators are completed. Several approaches are proposed to widen the lock range of non-harmonic oscillators, making them particularly attractive for applications such as PWMs where the accuracy of the frequency generated from the local oscillator is heavily affected by PVT. The contributions are listed as below:

1. Formalized the intrinsic relation between the lock range and the injection-induced variation of the impedance of LC tank.

2. Explored the mechanism of enlarging the ILAIOs.

3. Proposed a simple but effective approach to analyze the injection-locked oscillators.

4. Proposed a new approach to widen the lock range of the injection-locked non-harmonic oscillators.

5. Formalized the impacts on the lock range from different phase and tones of injections.

Extensive simulation results associated with the injection-locked active inductors and non-harmonic oscillators are presented.

\subsubsection{Tunable Band-Pass Filter Embedded Charge-Domain Sampling Circuits}

A novel sampling mixer with programmable centre frequency of the band-selection

filter and $-3 \mathrm{~dB}$ frequency of the anti-aliasing filters are proposed, providing a large freedom 
of selection of any frequency band signals without using discrete components. The timeinterleaved structure are utilized to achieve the optimal aliasing rejection. And the $-3 \mathrm{~dB}$ frequency is controlled by the sampling clock. The contributions include:

1. Proposed a novel architecture for software-defined radio.

2. Proposed a high-order embedded FIR filter in the sampling circuit.

3. Completed a in-depth study of periodical N-path sampling and charge-domain sampling.

4. Provided a unique noise analysis for the new architecture.

The detailed mathematical analysis and circuit simulation results of the proposed circuits are given. The layout and on-chip wafer measurement results of the circuits are provided.

\subsection{Thesis Organization}

The rest of the thesis is organized as follows:

- Chapter 2 presents a systematic study for the injection-locked active inductor oscillators. In the mean time, an novel approach to analyze the lock range of the injection-locked oscillators is also provided. The relation between the lock range and the impedance variation of active inductor oscillators is given.

- Chapter 3 presents a detailed investigations on injection-locked non-harmonic oscillators. The lock range of multiple multi-tone injection-locked oscillators is formalized. An example, relaxation oscillator, is used to verify the findings.

- Chapter 4 presents a low-power tunable band-pass filter embedded quadrature downconversion sampling circuit for SDR. In this chapter, mathematical derivation for the sampling network is provided and circuit implementation is shown. 
- Chapter 5 presents an alternative charge-domain sampling circuit with high-order sinc filter embedded. The circuit implementation is given, accompanied with simulations and measurements.

- Chapter 6 draws conclusions and brings some future research directions. 


\section{Chapter 2}

\section{Injection-Locked Active Inductor Oscillators}

In this chapter, the lock range of injection-locked active inductor oscillators is derived in analogous to the analysis of oscillators in a linear feedback system. Making use of this novel approach, the relation between injection ratio and impedance variation of injectionlocked oscillators and lock range is analyzed analytically. The remaining of the chapter is organized as the follows: This chapter starts from introduction of injection-locked oscillators in Section 2.1. It is followed by a brief description of active inductor oscillators in Section 2.2. A study of lock range for LC tank oscillators is given in Section 2.3. Section 2.4 explores the intrinsic relation between lock range and impedance variation of injection-locked oscillators. A case study is presented in Section 2.5. A summary is provided in Section 2.6.

\subsection{Injection-Locked Oscillators}

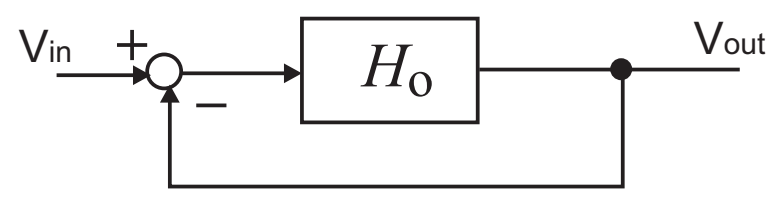

Figure 2.1: A feedback system.

A typical feedback system with unity loop gain is illustrated in 2.1, the transfer function is given as 


$$
\frac{V_{\text {out }}}{V_{\text {in }}}(j \omega)=\frac{H_{o}(j \omega)}{1+H_{o}(j \omega)}
$$

Unlike an amplifier with negative feedback, which is often to set enough phase margin at the unity-gain frequency to make the feedback stable, in the self-sustaining autonomous system, a unstable negative feedback is desired. From 2.1, if $H_{o}\left(j \omega_{o}\right)=-1$, the loop gain approaches infinity. A noise component at $\omega_{o}$ experiences a total gain of unity and a phase shift of $180^{\circ}$, returning to the subtractor as a reversed input signal. The output of subtractor becomes larger and continuous to grow. As a result, an oscillating signal is obtained. In general, if a negative-feedback system with loop gain satisfies "Barkhausen criteria".

$$
\begin{array}{r}
\left|H_{o}\left(j \omega_{o}\right)\right| \geq 1 \\
\angle H_{o}\left(j \omega_{o}\right)=180^{\circ}
\end{array}
$$

the feedback system may oscillate at $\omega_{o}$.

In an ideal "LC tank" circuit, a charged capacitor is connected to an inductor, this charge will flow back and forth between the inductor and capacitor, causing voltage across the capacitor to oscillate at the frequency of $\omega_{o}=1 / \sqrt{L C}$. In other words, the impedance of the inductor and the capacitor are equal and opposite, resulting open-circuit at $\omega_{o}$ ideally. In reality, real inductor and capacitor are always associated with parasitic resistances in which partial energy is absorbed, yielding a decayed waveform. To maintain oscillation, negative resistances are placed in the tank to compensate the parasitic resistances. Such a circuit configuration is given a name as cross-coupled LC oscillator [91]. If an incident signal, $I_{i n j}$, operating at $\omega_{i n j}$, is injected into the tank, the tank is under perturbation, which is so called injection process, as shown in Fig. 2.2.

As the injection signal is increased from weak to relatively strong, and the frequency of the injection signal is close enough to $\omega_{0}$, the output of the oscillator is starting to be 


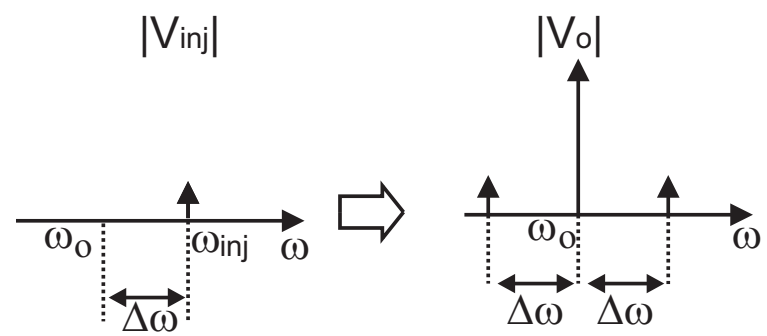

(a)

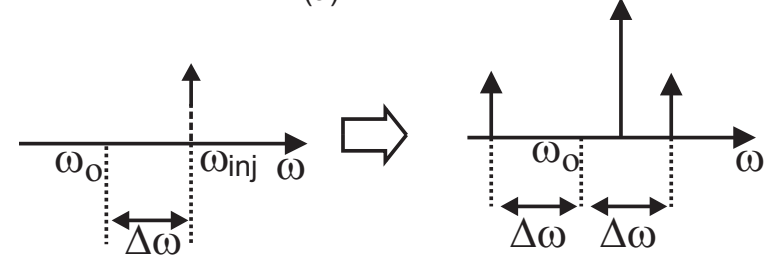

(b)

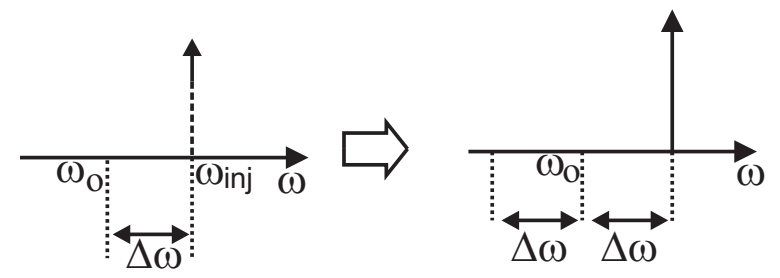

(c)

Figure 2.2: Injection locked procedure represented in simplified spectrum diagram. (a)Unlocked, (b) Fast beat, and (c) Locked.

pulled towards the injection signal and eventually is synchronized with it. The condition for the synchronization was first examined by Alder [61] in 1946.

$$
\frac{E_{1}}{E}=2 Q \frac{\Delta \omega}{\omega_{0}} .
$$

Where $E_{1}$ and $E$ are the impressed voltage and the oscillating voltage respectively. Q denotes the quality factor of the tank. $\omega_{0}$ and $\Delta \omega$ are free-running frequency and the maximum difference between the frequency of the injection signal and the free-running frequency of the oscillator over which a lock state can be established. Although the pioneering work of Adler on injection-locking in oscillators has been widely accredited, the phasor domain treatment of the injection-locking and pulling of oscillators by Razavi provides the much 
needed insight of the locking and pulling processes of oscillators under injection [53].

$$
\Delta \omega=\frac{\omega_{o}}{2 Q} \frac{I_{i n j}}{I_{o}} \frac{1}{\sqrt{1-\left(\frac{I_{i n j}}{I_{o}}\right)^{2}}}
$$

where $I_{i n j}$ is the amplitude of the injection current, and $I_{o}$ is the amplitude of the current of the LC tank. If $I_{i n j} \ll I_{o}$, i.e. weak injection, (2.4) is simplified to

$$
\Delta \omega \approx \frac{\omega_{o}}{2 Q} \frac{I_{i n j}}{I_{o}}
$$

It is seen from (2.5) that the lock range of the oscillator is directly proportional to the strength of the injection signal and inversely proportional to the current and quality factor of the LC tank.

\section{$2.2 \quad$ Active Inductor Oscillators}

CMOS active inductors are active networks that consist mainly of MOS transistors. As compared with their spiral counterparts, CMOS active inductors offer many advantages, e.g. a large amount of silicon area saving, large and tunable inductance and more compatibility with digital CMOS technologies [92]. Passive capacitors may be added in the circuits to change frequency. Sometimes resistors are used as feedback elements to improve performance of active inductors. When the active networks are biased properly, the networks exhibit an inductive characteristic at a specific frequency range. Fig. 2.3 shows an active inductor network, where $C_{1}$ and $G_{o 1}, C_{2}$ and $G_{o 2}$ denote the total capacitances and conductances at nodes 1 and 2 respectively.

The admittance looking into port 2 of the network is shown as: 

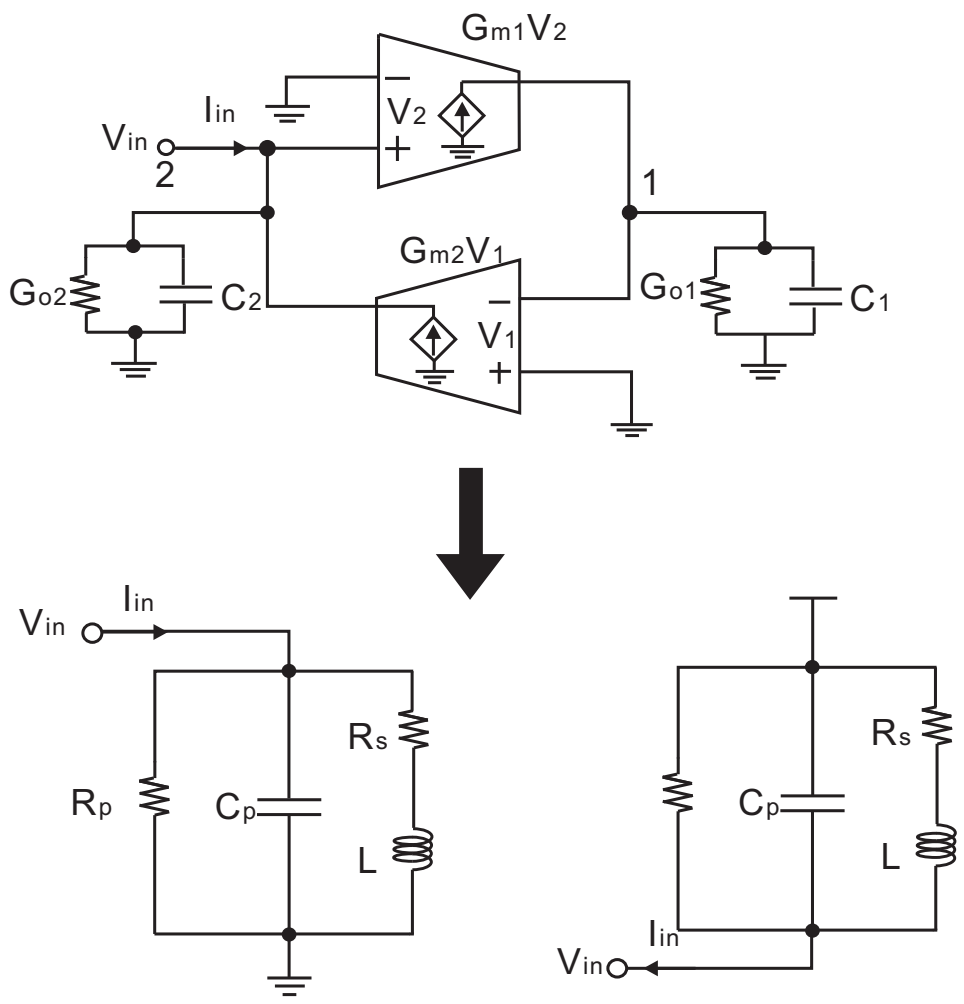

Figure 2.3: Single-ended active inductor.

$$
\begin{aligned}
Y & =\frac{I_{i n}}{V_{2}} \\
& =s C_{2}+G_{o 2}+\frac{1}{s\left(\frac{C_{1}}{G_{m 1} G_{m 2}}\right)+\frac{G_{o 1}}{G_{m 1} G_{m 2}}} .
\end{aligned}
$$

(2.6) can be represented by equivalent RCL circuit in Fig. 2.3 with its parameters given by

$$
\begin{aligned}
& R_{p}=\frac{1}{G_{o 2}} \\
& C_{p}=C_{2} \\
& R_{s}=\frac{G_{o 1}}{G_{m 1} G_{m 2}} \\
& L=\frac{C_{1}}{G_{m 1} G_{m 2}} .
\end{aligned}
$$


It can see from (2.7), the resonant frequency is $\omega_{0}=\sqrt{\left(G_{m 1} / C_{1}\right)\left(G_{m 2} / C_{2}\right)}$. And also the tuning range of the inductance is determined by $C_{1}, G_{m 1}$ and $G_{m 2}$, which in general $G_{m 1,2}$ is controlled by DC biasing voltage. Fig. 2.4 lists some of examples. In Fig. 2.4(a), Wu current reuse active inductor will be used to construct an oscillator for the rest of study [93]. Fig. 2.4(b) use NMOS and PMOS to form an inductor [94]. The simplest active inductor [95] is illustrated in Fig. 2.4(c).

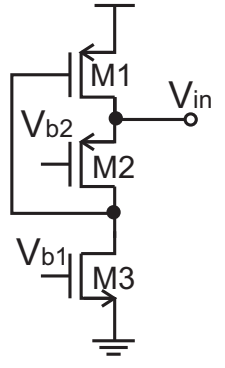

( a )

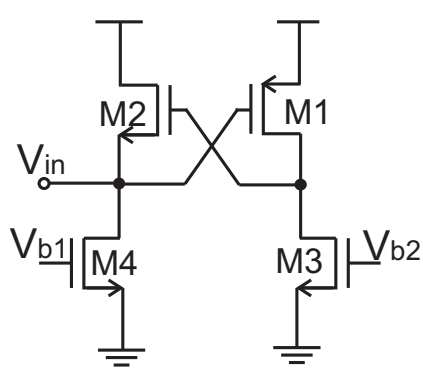

( b )

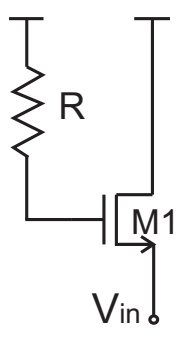

( C )

Figure 2.4: Examples of active inductors. (a) Wu current reuse active inductor , (b) Lin active inductor, and (c) Hara active inductor

\subsection{Lock Range of Generic LC Oscillators}

Consider the simplified schematic of an injection-locked LC oscillator shown in Fig. 2.5(a). The inverting buffer is needed in order to satisfy Barkhausen criteria. Let $I_{i n j}$ denote the injection signal and $Z_{T}$ denote the impedance looking into the LC tank. Since $I_{o}+I_{T}=I_{i n j}$ and $I_{o}=-g_{m} Z_{T} I_{T}$ where $g_{m}$ is the transconductance of the transistor, the injection-locked oscillator can be represented by the familiar block diagram used in control systems shown in Fig. 2.5(b) with $I_{i n j}$ the input, $I_{T}$ the output. Note that Fig. 2.5(b) is a linear system when locked. When the injection signal is absent, the oscillator is an autonomous system that oscillates at $\omega_{o}$, the self-resonance frequency of the LC tank.

When a sinusoidal signal of frequency $\omega_{i n j}=\omega_{o}+\Delta \omega$ is injected and the oscillator is locked to the injection signal, it will oscillate at $\omega_{o}+\Delta \omega$. Since $\omega_{i n j} \neq \omega_{o}$, the impedance of 


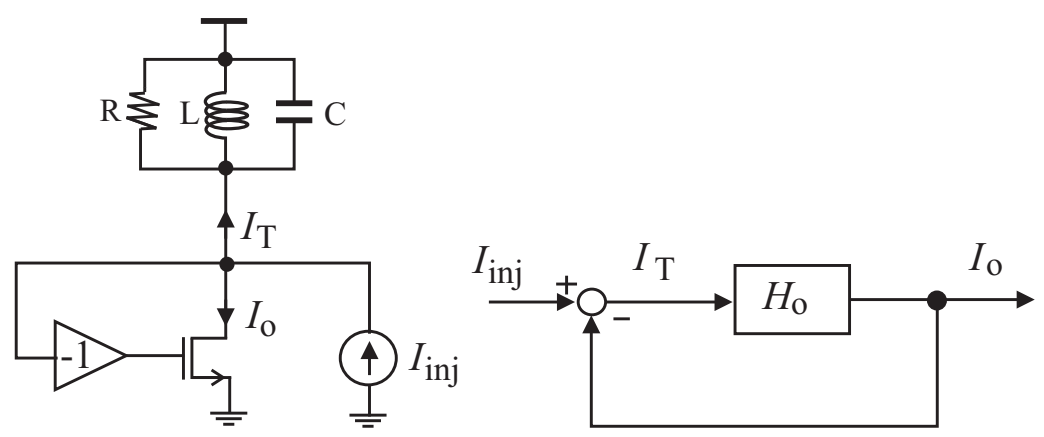

(a)

(b)

Figure 2.5: (a) Injection-locked oscillators. (b) Block diagram of injection-locked oscillators.

the LC tank at $\omega_{o}+\Delta \omega$ will deviate from its value at $\omega_{o}$, as illustrated graphically in Fig. 2.6. Clearly

$$
Z_{T}\left(\omega_{o}+\Delta \omega\right)=Z_{T}\left(\omega_{o}\right)-\Delta Z_{T}
$$

where $\Delta Z_{T}$ is the injection-induced impedance variation of the LC tank. Since $\Delta \omega$ is caused by $I_{i n j}, \Delta Z_{T}$ is also a function of $I_{i n j}$. Further, since $\Delta \phi$ and $\Delta Z_{T}$ are determined by Barkhausen criteria for oscillation, the same amount of phase and impedance variation is required to satisfy Barkhausen criteria regardless whether the quality factor of the LC tank is high or low. As a result, for the same injection signal, the high the quality factor of the LC tank, the smaller the frequency variation $\Delta \omega$, as illustrated graphically in Fig. 2.6.

Now let us consider the control system of Fig. 2.5(b). When the oscillator is locked to the injection signal, it will oscillate at $\omega_{o}+\Delta \omega$ and

$$
I_{o}\left(\omega_{o}+\Delta \omega\right)=\frac{H_{o}\left(\omega_{o}+\Delta \omega\right)}{1+H_{o}\left(\omega_{o}+\Delta \omega\right)} I_{i n j}\left(\omega_{o}+\Delta \omega\right)
$$

Note that in (2.9) the oscillating frequency is explicitly stated as we are only interested in the behavior of the oscillator in the lock state. Assume that $\Delta \omega \ll \omega_{o}$, which is often the 


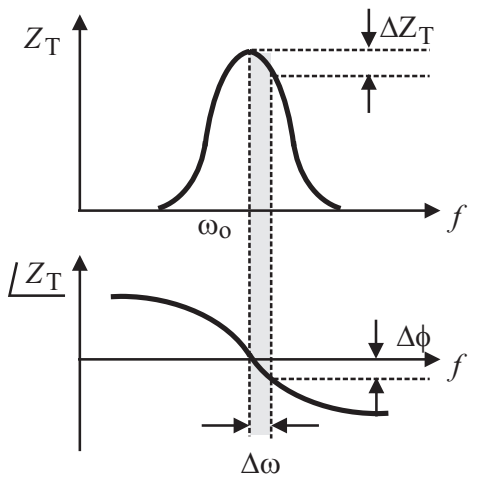

(a) High Q

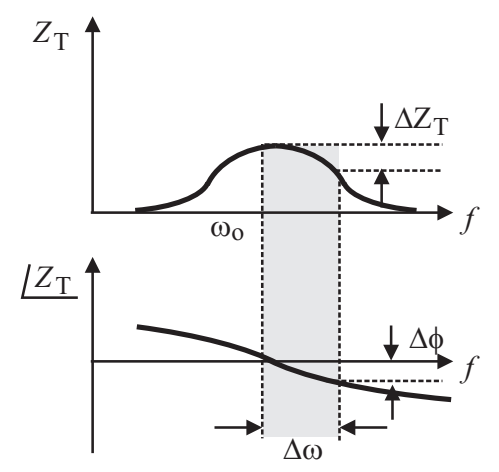

(b) Low Q

Figure 2.6: Impedance of the LC tank of injection-locked LC oscillators.

case, the first-order approximation

$$
H_{o}\left(\omega_{o}+\Delta \omega\right) \approx H_{o}\left(\omega_{o}\right)+\left[\frac{\partial H_{o}(\omega)}{\partial \omega}\right]_{\omega_{o}} \Delta \omega
$$

can be utilized to simplify (2.9)

$$
I_{o}\left(\omega_{o}+\Delta \omega\right) \approx \frac{H_{o}\left(\omega_{o}+\Delta \omega\right)}{1+H_{o}\left(\omega_{o}\right)+\left[\frac{\partial H_{o}(\omega)}{\partial \omega}\right]_{\omega_{o}} \Delta \omega} I_{i n j}\left(\omega_{o}+\Delta \omega\right) .
$$

The first-order approximation is only valid if the injection is weak. Since the injection signal is absent, $1+H_{o}\left(\omega_{o}\right)=0$, i.e. Barkhausen criteria, (2.11) is simplified to

$$
I_{o}\left(\omega_{o}+\Delta \omega\right) \approx \frac{H_{o}\left(\omega_{o}+\Delta \omega\right)}{\left[\frac{\partial H_{o}(\omega)}{\partial \omega}\right]_{\omega_{o}} \Delta \omega} I_{i n j}\left(\omega_{o}+\Delta \omega\right)
$$

Focusing on the amplitude only

$$
\left|I_{o}\left(\omega_{o}+\Delta \omega\right)\right| \approx \frac{\left|H_{o}\left(\omega_{o}+\Delta \omega\right)\right|}{\left|\frac{\partial H_{o}(\omega)}{\partial \omega}\right|_{\omega_{o}}|\Delta \omega|}\left|I_{i n j}\left(\omega_{o}+\Delta \omega\right)\right|
$$


Given $H_{o}(\omega)=A e^{j \phi}$, we have

$$
\frac{\partial H_{o}(\omega)}{\partial \omega}=\left(\frac{\partial A}{\partial \omega}+A j \frac{\partial \phi}{\partial \omega}\right) e^{j \phi}
$$

As $\left|A\left(\omega_{o}\right)\right|=1$ because of $1+H_{o}\left(\omega_{o}\right)=0$,

$$
\left|\frac{\partial H_{o}(\omega)}{\partial \omega}\right|_{\omega_{o}}=\sqrt{\left(\frac{\partial A}{\partial \omega}\right)^{2}+\left(\frac{\partial \phi}{\partial \omega}\right)^{2}}
$$

Making use of the definition of the quality factor [96]

$$
Q=\frac{\omega_{o}}{2} \sqrt{\left(\frac{\partial A}{\partial \omega}\right)_{\omega_{o}}^{2}+\left(\frac{\partial \phi}{\partial \omega}\right)_{\omega_{o}}^{2}}
$$

We arrive at

$$
\left|\frac{\partial H_{o}(\omega)}{\partial \omega}\right|_{\omega_{o}}=\frac{2 Q}{\omega_{o}}
$$

Substituting (2.17) into (2.13)

$$
\left|I_{o}\left(\omega_{o}+\Delta \omega\right)\right| \approx \frac{\left|H_{o}\left(\omega_{o}+\Delta \omega\right)\right|}{\frac{2 Q}{\omega_{o}}|\Delta \omega|}\left|I_{i n j}\left(\omega_{o}+\Delta \omega\right)\right|
$$

Further from Fig. 2.5,

$$
\left|I_{o}\left(\omega_{o}+\Delta \omega\right)\right|=\left|H_{o}\left(\omega_{o}+\Delta \omega\right)\right|\left|I_{T}\left(\omega_{o}+\Delta \omega\right)\right|
$$

equating (2.18) and (2.19) yields

$$
|\Delta \omega|=\frac{\omega_{o}}{2 Q}\left|\frac{I_{i n j}\left(\omega_{o}+\Delta \omega\right)}{I_{T}\left(\omega_{o}+\Delta \omega\right)}\right|
$$


It is of interest to comment above results :

1. When the injection is weak, i.e., $I_{i n j} \ll I_{o}, I_{o} \approx I_{T}$ follows. As a result, (2.20) is the same as (2.5). Note that the latter was derived in a simple but efficient way in comparison with the approaches used in the previous published papers.

2. The preceding results are derived on the condition that the oscillator is locked to the injection signal. $|\Delta \omega|$ quantifies the maximum frequency deviation from $\omega_{o}$ upon which lock holds. It is therefore the lock range of the oscillator for given $I_{i n j}$.

3. (2.20) is only valid when (2.10) holds. This typically occurs when the amplitude of the injection signal is small with respect to the $I_{o}$. This agrees well with the condition upon which (2.5) was derived.

4. Since $|\Delta \omega|=\left|\omega_{i n j}-\omega_{o}\right|$, the lock range is only symmetrical if $I_{T}\left(\omega_{o}+\Delta \omega\right)=I_{T}\left(\omega_{o}-\right.$ $\Delta \omega)$, otherwise, asymmetric lock ranges exist. As the ratio of injection current to oscillator current increases, this condition will no longer be valid. This is especially true for low-Q oscillators, such as active-inductor oscillators [97]

5. A small $Q$ will result in a large lock range. This echoes early findings on the relation between the impedance variation and $Q$ of the LC tank in Fig. 2.6.

\subsection{Lock Range and Tank Impedance Variation}

It was pointed out earlier that the injection signal causes the variation of the impedance of the tank. This observation reveals that an intrinsic relation between the injection signal and the variation of the impedance of the resonant tank exists. To quantify the relation between the injection signal and the variation of the impedance of the tank, consider Fig. 2.7. In Fig. 2.7(a), a current $I_{i n j}$ of frequency $\omega_{o}+\Delta \omega$ is injected to node 2 of the oscillator. The oscillator is locked to the injection signal and oscillates at $\omega_{o}+\Delta \omega$. In Fig. 2.7(b), the effect of the injection signal on the oscillator is represented by the variation of the impedance of the LC tank (assume $g_{m}$-cell is constant). Note that Fig. 2.7(a) is a forced oscillator while 
Fig. 2.7(b) is a free-running oscillator, in which the impact of the injection signal has been merged into the LC tank, denoting by $\delta Z$.

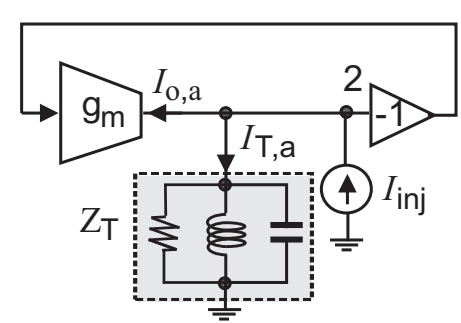

(a)

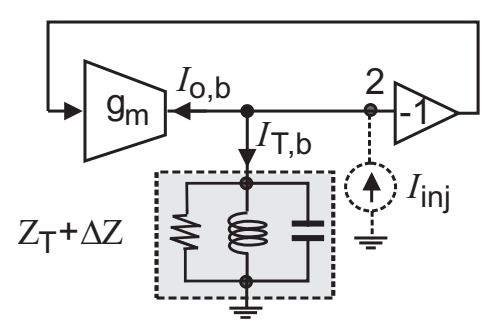

(b)

Figure 2.7: (a) Injection-locked oscillators. (b) Equivalent circuit of injection-locked oscillators. The dotted line represents the real injection signal, which has been merged into the variation of the LC tank.

Fig. 2.7(a) and Fig. 2.7(b) are said to be equivalent only if the voltages at node 2 of the two systems are identical. Since $V_{2, a}=Z_{T}\left(I_{i n j}-I_{o, a}\right)$ and $V_{2, b}=\left(Z_{T}+\Delta Z_{T}\right)\left(-I_{o, b}\right)$ where $Z_{T}$ is evaluated at $\omega_{o}+\Delta \omega$, from $V_{2, a}=V_{2, a}$, we have $I_{o, a}=I_{o, b}=I_{o}$. As a result,

$$
\left|\frac{\Delta Z_{T}}{Z_{T}}\right|=\left|\frac{I_{i n j}\left(\omega_{o}+\Delta \omega\right)}{I_{T}\left(\omega_{o}+\Delta \omega\right)}\right| .
$$

(2.21) reveals that the effect of the injection signal can be quantified by the variation of the impedance of the tank. Substituting (2.21) into (2.20) yields

$$
|\Delta \omega|=\frac{\omega_{o}}{2 Q}\left|\frac{\Delta Z_{T}}{Z_{T}}\right| .
$$

The preceding results are commented as :

1. For passive LC oscillators, since the quality factor of varactors is typically much higher(minimum Q is 80 in IBM CMRF8SF $130 \mathrm{~nm}$ technology) as compared with that of spiral inductors(maximum $Q$ of differential inductor is 25 in IBM CMRF8SF $130 \mathrm{~nm}$ technology), the quality factor of passive LC tanks is dominated by that of the spiral inductors. Since passive LC tanks exhibit a large quality factor as compared 
with that of active inductors, injection-locked passive LC oscillators have a small lock range while that of active-inductor oscillators have a large lock range.

2. The larger the normalized variation of the tank impedance induced by the injection signal, the larger the lock range. For spiral LC oscillators, since the effect of the injection signal on the capacitance of the varactors of these oscillators is small, $\Delta Z_{T} / Z_{T}$ is small as well. As a result, the lock range of these oscillators is small. This is in addition to the effect of the high quality factor of passive LC tanks, which also gives rise to a small lock range.

3. The inductance of active inductors varies with the injection signal especially when the injection signal is strong, $\Delta Z_{T} / Z_{T}$ of active inductors is large. As a result, activeinductor oscillators exhibit a large lock range. This is on top of the effect of the low quality factor of active inductors, which contributes to the large lock range of these oscillators.

4. The symmetry of the lock range is clearly determined by the symmetry of $\left|\Delta Z_{T}\right|$. Since active inductors are gyrator-based whose operating points are bias-dependent and injection signals affect the bias conditions, active-inductor oscillators will exhibit more asymmetrical lock ranges.

5. For passive LC oscillators, an injection signal will cause the capacitor of the varactors to change. For active-inductor oscillators, it is the inductance of the active inductors that will change with the injection signal.

Consider an LC tank with its $C$ changed to $C+\Delta C$. Let $Z_{R L}=R \| s L$, where " "| denotes parallel connection. Since $\hat{X}_{c}=1 / s(C+\Delta C)=X_{c}-\Delta X_{c}$, where $\Delta X_{c}=X_{c}(\Delta C / C)$ and $X_{c}=1 / s C$. The impedance of the tank is obtained from

$$
\hat{Z_{T}} \approx Z_{T}\left[1+\frac{Z_{R L}}{\left(Z_{R L}+X_{c}\right) X_{c}} \Delta X_{c}\right]
$$


from which we arrive at

$$
\Delta Z_{T} \approx \frac{Z_{R L} Z_{T}}{\left(Z_{R L}+X_{c}\right) X_{c}} \Delta X_{c} .
$$

Note that first-order approximation was utilized in derivation of (2.23). In a similar manner, one can show that when $L$ is changed to $L+\Delta L$, we have

$$
\Delta Z_{T} \approx \frac{Z_{R C} Z_{T}}{\left(Z_{R C}+X_{L}\right) X_{L}} \Delta X_{L}
$$

where $Z_{R C}=R \|(1 / s C), X_{L}=s L$, and $\Delta X_{L}=X_{L}(\Delta L / L)$. The preceding results demonstrate that $\Delta Z_{T}$ is directly related to $\Delta C$ or $\Delta L$.

\subsection{Comparison of Injection-Locked LC Oscillators and Active Inductor Oscillators}

In this section, it will be given that $\Delta C$ of injection-locked passive LC oscillators or $\Delta L$ of injection-locked active-inductor LC oscillators are indeed caused by the injection signal. Two oscillators are designed running at $2.4 \mathrm{GHz}$ for the comparison purpose.

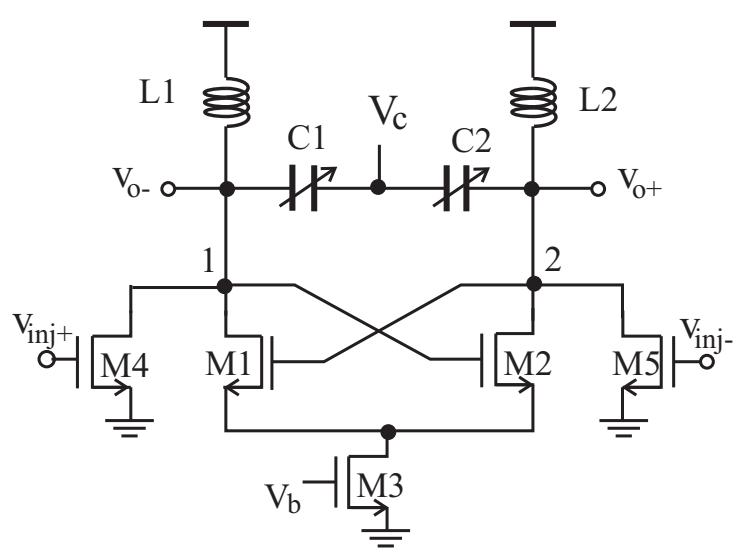

Figure 2.8: Injection-locked passive $\mathrm{LC}$ oscillator. $C_{1}$ and $C_{2}$ are accumulation MOS varactors. Circuit parameters : $W_{1,2}=10 \mu \mathrm{m} ; W_{3}=15 \mu \mathrm{m}, W_{4,5}=1 \mu \mathrm{m}, V_{c}=1.2 \mathrm{~V}, V_{b}=0.66 \mathrm{~V}$, $I_{d s 3}=1.42 \mathrm{~mA}$. 


\section{Passive LC Oscillators}

Consider the injection-locked passive LC oscillator in Fig. 2.8. Assume that the variation of the capacitance at nodes 1 and 2 is dominated by change of the capacitance of the varactors. Let the voltage across varactor $C_{1}$ be $V_{c 1}=V-V_{c}+\Delta V$, where $V$ is the dc voltage of node 1 , and $\Delta V$ is the variation of the voltage across $C_{1}$ due to both injection and oscillation. Similarly, the voltage across $C_{2}$ is given by $V_{c 2}=V-V_{c}-\Delta V$. The corresponding capacitances of varactors 1 and 2 are given by $C_{1}=C+\Delta C_{\text {osc }}+\Delta C_{\text {inj }}$ and $C_{2}=C-\Delta C_{o s c}-\Delta C_{i n j}$, where $C$ is the capacitance of the varactors in the de steady state, $\Delta C_{\text {osc }}$ is the oscillation-inducted capacitance variation and $\Delta C_{i n j}$ is the injection-induced capacitance variation. Assume $\Delta C_{o s c}, \Delta C_{i n j} \ll C$. The capacitance between nodes 1 and 2 , denoted by $C_{12}$, is obtained from

$$
C_{12}=\frac{C}{2}\left[1-\frac{\left(\Delta C_{o s c}+\Delta C_{i n j}\right)^{2}}{C^{2}}\right] .
$$

From (2.26), it can be seen that the parentheses term indicates the variation of the overall capacitance due to the injection signal and oscillation. Since the capacitance looking into the negative resistor network and the capacitance of the injection transistors are rather constant, the total capacitance variation at nodes 1 and 2 is largely reduced, resulting in a narrow lock range.

\section{Active Inductor Oscillators}

Consider the injection-locked active inductor oscillator in Fig. 2.9 that employs Wu currentreuse active inductors reported and shown in the shade area. Wu active inductor can be represented by an RLC equivalent circuit with $C_{p}=C_{g s 2}, R_{p}=1 / g_{m 2}, R_{s}=\left(g_{o 2}+\right.$ $\left.g_{o 3}\right) /\left(g_{m 1} g_{m 2}\right)$, and $L=C_{g s 1} /\left(g_{m 1} g_{m 2}\right)$, where $g_{m}$ and $g_{o}$ are the transconductance and output conductance of $\mathrm{M} 1$, respectively [92]. It is evident that $L, R_{p}$, and $R_{s}$ of the active inductor can be varied by adjusting the current of M1 and M2.

Since the current of M2 is constant, it is reasonable to assume that $g_{m 2}$ is constant. As a result, $V_{g, 2}=V_{s, 1}$ are also constant. $g_{m 1}$, however, varies with both oscillation and the 


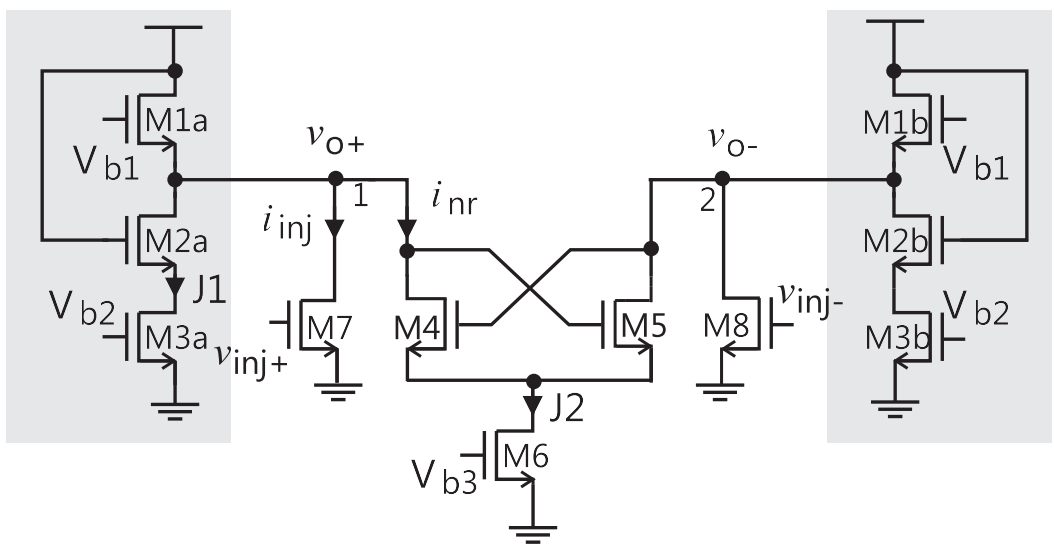

Figure 2.9: Schematic of injection-locked active-inductor VCO with $\mathrm{Wu}$ active inductors. Circuits parameters $: W_{1 a, 1 b}=40 \mu \mathrm{m}, W_{2 a, 2 b}=18.9 \mu \mathrm{m}, W_{3 a, 3 b}=4 \mu \mathrm{m}, W_{4,5}=10 \mu \mathrm{m}, W_{6}=$ $15 \mu \mathrm{m}, W_{7,8}=1 \mu \mathrm{m}, V_{b 1}=0.34 \mathrm{~V}, V_{b 2}=0.48 \mathrm{~V}, V_{b 3}=0.7 \mathrm{~V}, I_{d s 3}=131 \mu \mathrm{A}, I_{d s 6}=1.42 \mathrm{~mA}$.

injection signal. Because $I_{D 1}=J_{1}+i_{i n j}+i_{n r}$, where $i_{n r}$ is the current of the negative resistor and $i_{i n j}$ is the injection current. Let $V_{T}=V_{T N}=\left|V_{T P}\right|$, we have

$$
g_{m 1}=g_{m 1, D C}\left(1+\frac{i_{i n j}}{J_{1}}+\frac{i_{n r}}{J_{1}}\right)
$$

where $g_{m 1, D C}=2 J_{1} /\left(V_{S G 1}-V_{T}\right)$ is the transconductance of M1 in the dc steady state. Let $i_{n r}^{+}=I+\Delta I$ and $i_{n r}^{-}=I-\Delta I$ where $I=J_{2} / 2$. The inductance of the active inductor is given by

$$
L_{1}=\frac{L_{D C}}{1+\frac{I}{J_{1}}}\left(\frac{1}{1+\frac{i_{i n j}+\Delta I}{J_{1}+I}}\right)
$$

where $L_{D C}=C_{g s 1} /\left(g_{m 1, D C} g_{m 2, D C}\right)$. Similarly,

$$
L_{2}=\frac{L_{D C}}{1+\frac{I}{J_{1}}}\left(\frac{1}{1-\frac{i_{i n j}+\Delta I}{J_{1}+I}}\right)
$$


Since active inductors $L_{1}$ and $L_{2}$ are connected in series, the inductance looking into nodes 1 and 2 is given by

$$
L_{12}=\frac{2 L_{D C}}{1+\frac{I}{J_{1}}}\left[1+\left(\frac{\Delta I+i_{i n j}}{J_{1}+I}\right)^{2}\right] \text {. }
$$

The variation of the overall inductance is due to the injection signal and the oscillation. Since $i_{i n j}$ directly affect $L_{12}$ while in the injection-locked passive LC oscillator, the injection current impacts the capacitance of the varactors through the voltage of nodes 1 and 2 , the injection signal will have a large impact on the inductance of the injection-locked active-inductor oscillator subsequently a large lock range.

The passive LC tank oscillator shown in Fig. 2.8 and the active-inductor LC oscillator shown in Fig. 2.9 are utilized to validate the results derived earlier. Simulations are done in Cadence SpectreRF. The incident power of injection signal for both oscillators is a $-18 \mathrm{dBm}$, the ratio of $I_{i n j} / I_{o}=0.052 .40205 \mathrm{GHz}$ sinusoid with phase noise $-126 \mathrm{dBc} / \mathrm{Hz}$ at $1 \mathrm{MHz}$ frequency offset.

Fig. 2.10 plots the phase noise of the passive LC oscillator. The phase noise of the oscillator without injection-locking is $-102 \mathrm{dBc} / \mathrm{Hz}$ at $1 \mathrm{MHz}$ frequency offset and -124 $\mathrm{dBc} / \mathrm{Hz}$ when locked.

Fig. 2.11 plots the phase noise of the active-inductor oscillator, simulated in Cadence Spectre. The phase noise of the oscillator without the injection-locking reference is $-70 \mathrm{dBc} / \mathrm{Hz}$ at 1 $\mathrm{MHz}$ frequency offset and is reduced to $-118 \mathrm{dBc} / \mathrm{Hz}$ when locked.

To provide a fair comparison of the lock range, the negative resistors of the oscillators are biased at the same level. The swing of the output voltage of the oscillators is also made identical. Fig. 2.12 is dependence of the lock range on the injection signal. It is observed that the lock range is directly proportional to the injection signal. This agrees well with the theoretical results. Also observed is that the lock range of injection-locked active inductor oscillator is much larger as compared with that of the injection-locked passive LC oscillator. To demonstrate the larger lock rang of the active inductor oscillator is not only due to the 


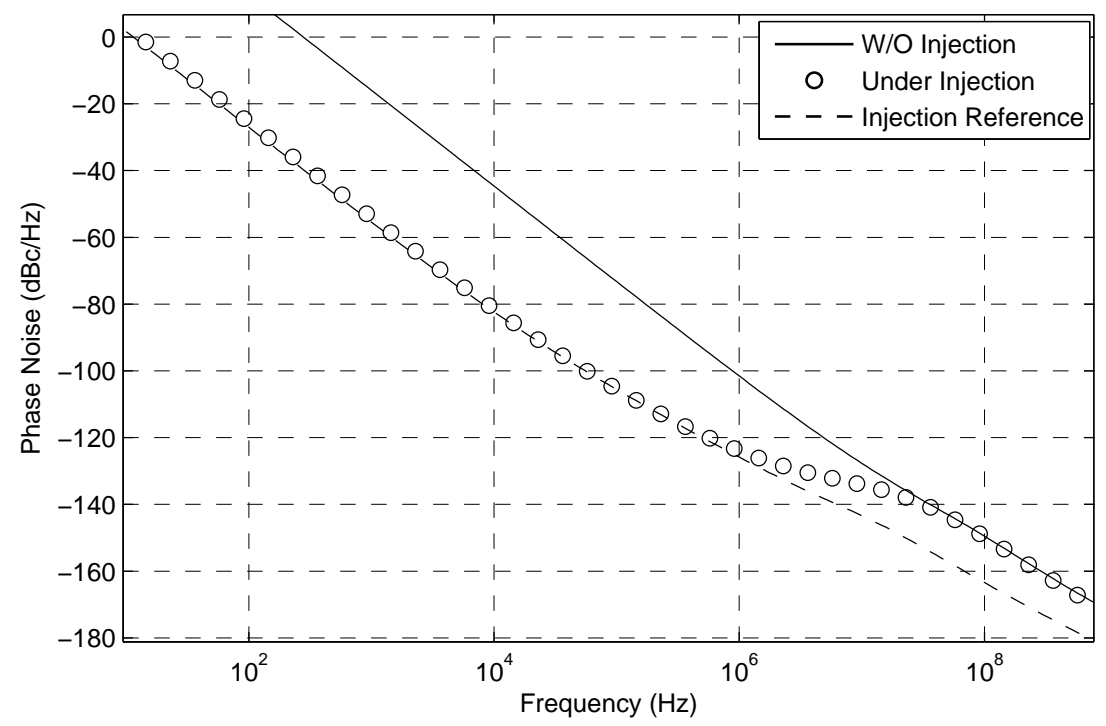

Figure 2.10: Simulated phase noise of passive LC oscillator $I_{i n j} / I_{O}=0.05$.

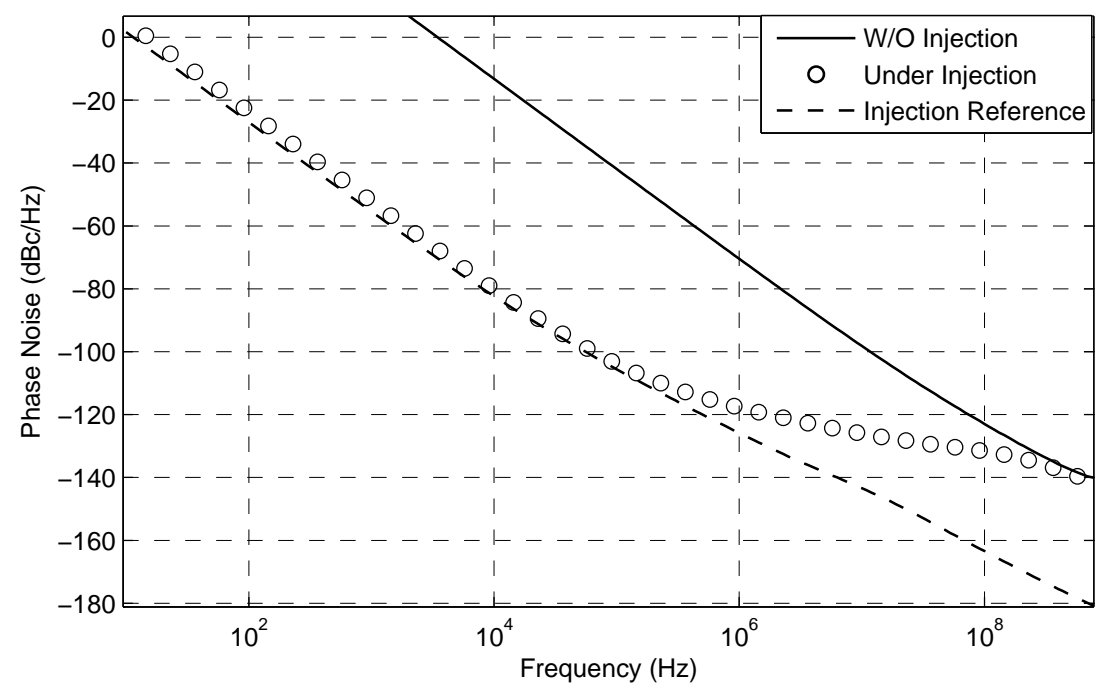

Figure 2.11: Simulated phase noise of active-inductor LC oscillator $I_{i n j} / I_{o}=0.05$.

low quality factor but injection-induced impedance variation, the re-sized passive oscillator by inserting series resistors to lower Q-factor, which is in the comparable level to the active inductor oscillator, is simulated. The lock range of passive one is still less than the active one. 


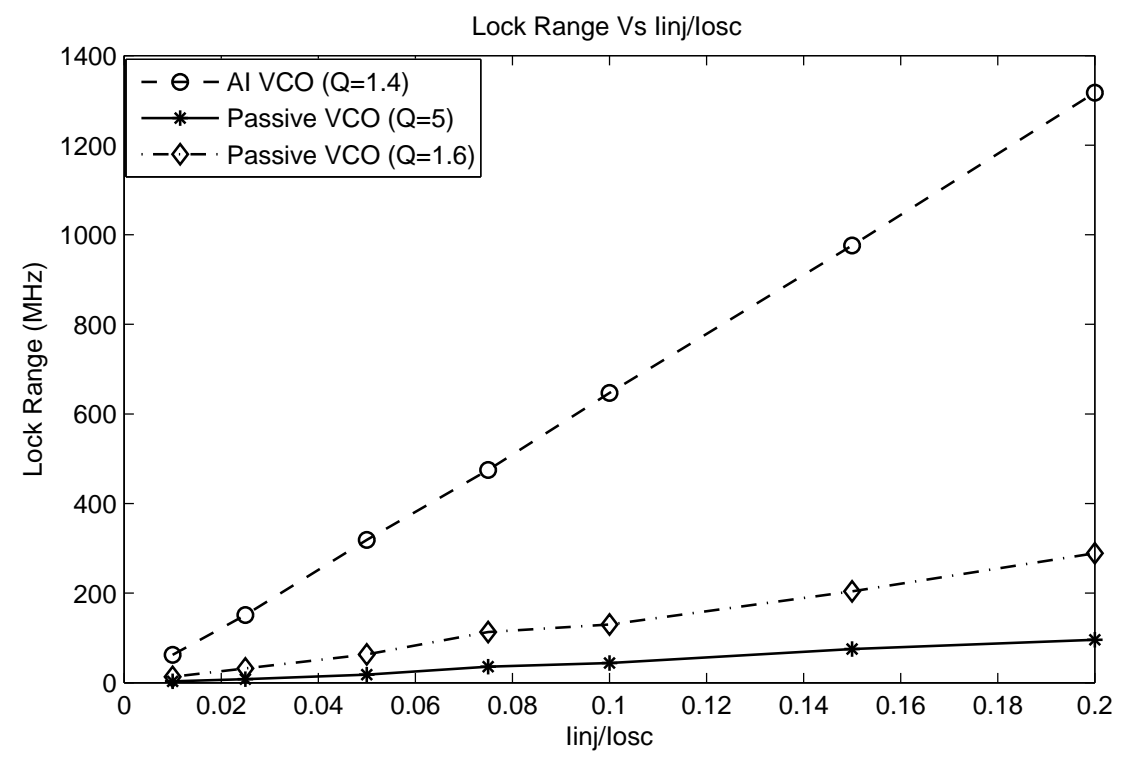

Figure 2.12: Simulated lock range.

Active inductor oscillators are low- $Q$ systems. When injection ratio increases, not only lock range will increase, the asymmetry of the lock range of these oscillators will also becomes more prominent. This is evident in Fig. 2.13 where the positive and negative lock ranges of the active inductor oscillator investigated in this paper are plotted. The asymmetry of the lock range is evident.

\subsection{Summary}

In this chapter, an in-depth investigation of the lock range of injection-locked LC oscillators using a control system approach has been presented. The intrinsic relation between the lock range of these oscillators and the injection-induced variation of the impedance of the LC tank of these oscillators has been explored. The large lock range of injection-locked active-inductor oscillators is attributed to both the low quality factor and the large injectioninduced variation of the inductance of active inductors. 


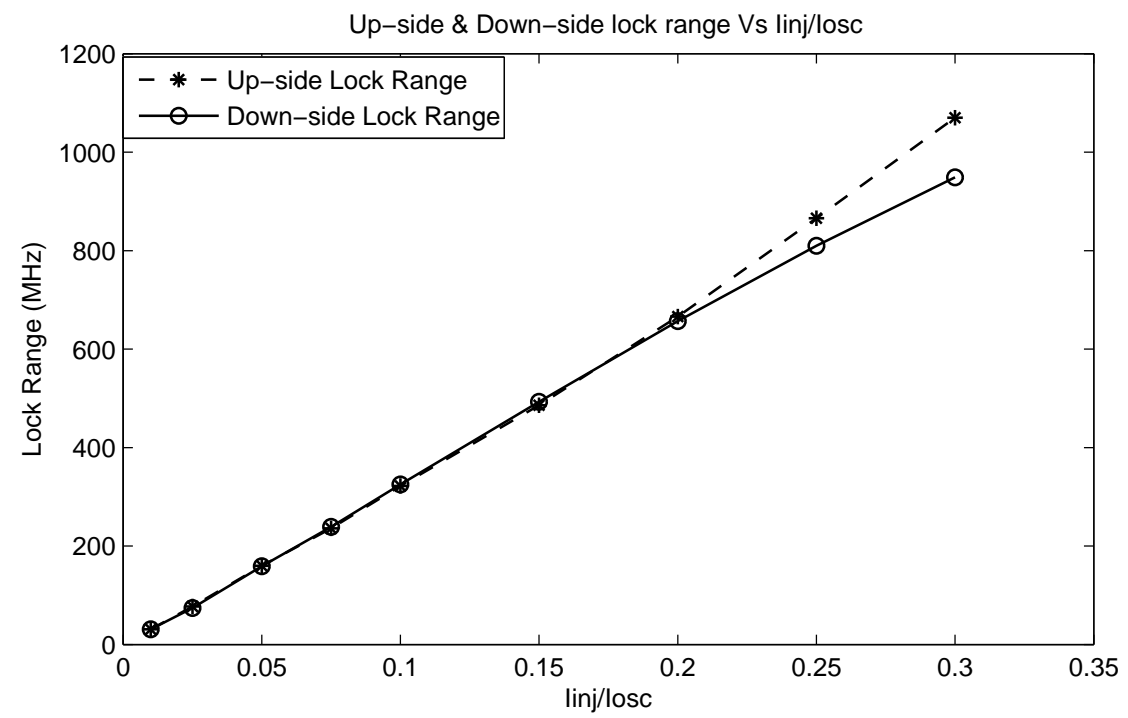

Figure 2.13: Asymmetry of the lock range of injection-locked active-inductor oscillator. 


\section{Chapter 3}

\section{Multiple Multi-Tone Injection-Locked Oscilla- tors}

In general, Volterra series can be used to analyze a non-linear behaviour. In this chapter, an approach similar to Volterra series is employed to explore the non-harmonic injection-locked oscillators. The oscillators can be analyzed by applying a set of linear circuits, having the same topology and element values but different inputs. The analytical study of the lock range is also given in detail. The remaining of the chapter is organized as the follows: Section 3.1 presents a simplified mode of injection-locked non-harmonic oscillators. Section 3.2 investigates the single-tone and multi-tone injections for non-harmonic oscillators. Section 3.3 gives an analysis for harmonic injection-locked oscillators with multiple injections. Section 3.4 examines the non-harmonic injection-locked oscillators with multiple injections. Section 3.5 shows the study of lock range of non-harmonic oscillators with multiple singletone injections. A relaxation oscillator is given as an example in Section 3.6. A study of lock range of non-harmonic oscillators with multiple multi-tone injections is presented in Section 3.7, followed by simulations in Section 3.8. A summary is provided in Section 3.9.

\subsection{Representation of Non-Harmonic Oscillators}

The waveform of the output voltage of LC oscillators is sinusoidal whereas that of non-harmonic oscillators is typically square-like. To capture the essence of non-harmonic oscillators, assume that the output of these oscillators is a purely square wave such that it 
can be represented by a Fourier series with the fundamental frequency $\omega_{o}=2 \pi / T_{o}$ where $T_{o}$ is the frequency of the oscillator. If the waveform of the output of the oscillator is shown in Fig.3.1(a), then its spectrum will only contain the fundamental and odd harmonics. Since the spectrum of the output of the oscillator only consists of a train of impulses at $\omega_{o}, 3 \omega_{o}$, $5 \omega_{o}, \ldots$, the oscillator can be considered as the assembly of ideal harmonic oscillators whose frequency spectrum only contains single tones at $\omega_{o}, 3 \omega_{o}, 5 \omega_{o}, \ldots$ When the noise and loss of the non-harmonic oscillator are considered, the square waveform of the non-harmonic oscillator is replaced with those shown in Fig.3.1(b). In this case, the impulses are replaced with skirts.

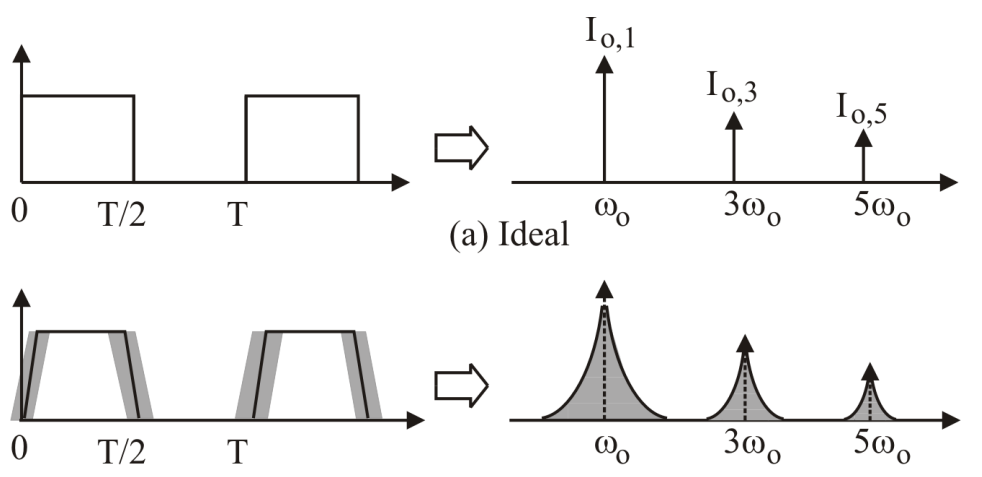

(b) Nonideal

Figure 3.1: Waveform and spectrum of non-harmonic oscillators.

Neglect the loss and noise of the oscillator, the output of the non-harmonic oscillator can be represented by

$$
I_{o}=\sum_{n=1,3, \ldots}^{\infty} I_{o, n}\left(n \omega_{o}\right) \delta\left(\omega-n \omega_{o}\right)
$$

where $\delta($.$) is the discrete-time impulse also known as the unit impulse [98]. Note that$ $\delta\left(\omega-\omega_{o}\right)=1$ if $\omega=\omega_{o}$ and 0 otherwise. The use of the discrete-time impulse enables us to conveniently capture the multi-tone characteristics of the spectrum of $I_{o}(t)$. 


\subsection{Representation of Injection-Locked Non-harmonic Oscillators}

\subsubsection{Single-Tone Injection}

When a single-tone signal $I_{i n j}(t)$, such as a sinusoid, is injected to a non-harmonic oscillator with its frequency within the lock range of the non-harmonic oscillator for the given injection strength, the frequency of the oscillator will be shifted from its free-running frequency $\omega_{o}$ to that of $I_{i n j}(t)$ and the oscillator is locked to $I_{i n j}$. If $I_{i n j}(t)$ is a single-tone at $\omega_{i n j}$, it can be represented by

$$
I_{i n j}(t)=I_{i n j} e^{j \omega_{i n j} t}
$$

When the non-harmonic oscillator is locked to $I_{i n j}(t)$, the frequency of the harmonic oscillators representing the non-harmonic oscillator are changed to $\omega_{i n j}, 3 \omega_{i n j}, \ldots$, accordingly. This observation reveals that the single-tone input of the non-harmonic oscillator $I_{i n j}(t)$ will affect the input of the representing harmonic oscillators. The injection-locked non-harmonic oscillator with a single-tone injection at $\omega_{i n j}$ can therefore be equivalent to a set of injectionlocked harmonic oscillators with injections at $\omega_{i n j}, 3 \omega_{i n j}, \ldots$, as shown in Fig. 3.2.

The preceding representation of an injection-locked non-harmonic oscillator with a set of injection-locked harmonic oscillators bears a strong resemblance to Volterra series analysis of nonlinear circuits. It is well understood that a nonlinear time-invariant circuit can be represented by a set of linear time-invariant circuits called Volterra circuits that have the same topology but distinct inputs determined by the nonlinearities of the circuits $[99,100]$. Similarly, it was demonstrated in $[101,102,103]$ that a periodically nonlinear time-varying circuit can be represented by a set of periodically linear time-varying circuits of the same topology but different inputs set by the nonlinearities of the circuits. In both cases, methods for analysis of linear systems such as Fourier transform can be applied to 


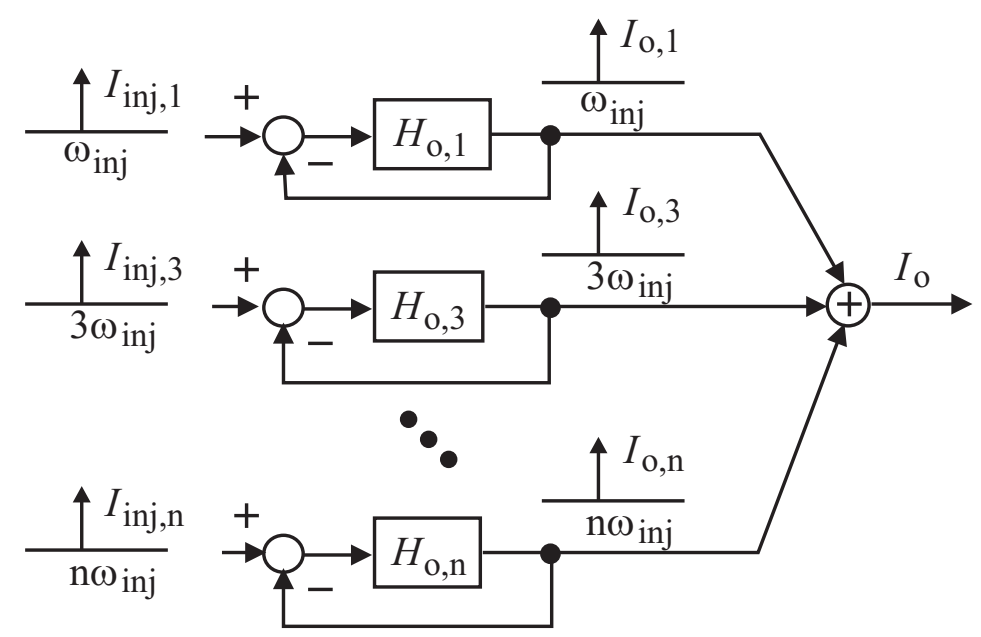

Figure 3.2: Representation of injection-locked non-harmonic oscillators with a single-tone injection.

analyze nonlinear circuits. As a result, non-harmonic oscillators fall into the category of periodically linear time-varying circuits.

\subsubsection{Multi-Tone Injection}

If the injection signal $I_{i n j}(t)$ is a multi-tone signal, such as a square wave of frequency $\omega_{i n j}$, it will contain multiple frequency components at frequencies $n \omega_{i n j}, n=1,3, \ldots$ (Assume $I_{i n j}(t)$ has the waveform shown in Fig. 3.1). In this case, $I_{i n j}(t)$ can be represented by

$$
I_{i n j}(t)=\sum_{n=1,3, \ldots}^{\infty} I_{i n j, n} e^{j n \omega_{i n j} t} .
$$

The impact of $I_{i n j}(t)$ on the oscillator can be analyzed by evaluating the effect of each frequency component of $I_{i n j}(t)$ individually in a similar way as that with a single-tone injection. This approach clearly is valid because although injection-locking is a nonlinear process, in the vicinity of the lock state the injection-locked non-harmonic oscillator can well be represented by a linear system. 
A short discussion associated with new definition of quality factor of harmonic oscillators and some of related equations, which will be used for the lock range study of multiple multi-tone injection-locked oscillators, are given as below. The definition of the quality factor of harmonic oscillators, (2.16), is extended to $n$ th-order harmonic oscillators representing the non-harmonic oscillator.

$$
Q_{n}=\frac{n \omega_{o}}{2} \sqrt{\left(\frac{\partial A_{n}}{\partial \omega}\right)_{n \omega_{o}}^{2}+\left(\frac{\partial \phi_{n}}{\partial \omega}\right)_{n \omega_{o}}^{2}} .
$$

Assume that the injection signal $I_{i n j}(t)$ causes the frequency of a non-harmonic oscillator to shift from its natural frequency $\omega_{o}$ to $\omega_{o}+\Delta \omega$ in the lock state with $\Delta \omega_{o} \ll \omega_{o}$ typically. If the injection is a single-tone at $\omega_{i n j}$, we will have $\omega_{i n j}=\omega_{o}+\Delta \omega$ in the lock state. If the injection signal is a multi-tone, such as a square wave, with its fundamental frequency $\omega_{i n j}$, $\omega_{i n j}=\omega_{o}+\Delta \omega$ will hold in the lock state. Using the first-order Taylor series approximation, we obtain the loop gain of $n$th harmonic oscillator

$$
H_{o, n}\left[n\left(\omega_{o}+\Delta \omega_{o}\right)\right] \approx H_{o, n}\left(n \omega_{o}\right)+\left(\frac{\partial H_{o, n}}{\partial \omega}\right)_{n \omega_{o}}(n \Delta \omega)
$$

Let $H_{o, n}=A_{n} e^{j \phi_{n}}$. Because

$$
\begin{aligned}
& \frac{\partial H_{o, n}}{\partial \omega}=e^{j \phi_{n}}\left[\frac{\partial A_{n}}{\partial \omega}+j A_{n} \frac{\partial \phi_{n}}{\partial \omega}\right] \\
& \frac{\partial H_{o, n}^{*}}{\partial \omega}=e^{-j \phi_{n}}\left[\frac{\partial A_{n}}{\partial \omega}-j A_{n} \frac{\partial \phi_{n}}{\partial \omega}\right]
\end{aligned}
$$

where the superscript $*$ denotes the complex conjugate operator, we have

$$
\frac{\partial H_{o, n}}{\partial \omega} \frac{\partial H_{o, n}^{*}}{\partial \omega}=\left(\frac{\partial A_{n}}{\partial \omega}\right)^{2}+A_{n}^{2}\left(\frac{\partial \phi_{n}}{\partial \omega}\right)^{2} .
$$


Evaluating (3.7) at $\omega=n \omega_{o}$ and noting that each harmonic oscillator satisfies Barhausen criteria

$$
1+H_{o, n}\left(n \omega_{o}\right)=0 \quad n=1,3,5, \ldots
$$

where $H_{o, n}\left(n \omega_{o}\right)$ is the loop gain of $n$th harmonic oscillator, we arrive at

$$
\left(\frac{\partial H_{o, n}}{\partial \omega} \frac{\partial H_{o, n}^{*}}{\partial \omega}\right)_{n \omega_{o}}=\left(\frac{\partial A_{n}}{\partial \omega}\right)_{n \omega_{o}}^{2}+\left(\frac{\partial \phi_{n}}{\partial \omega}\right)_{n \omega_{o}}^{2}
$$

Making use of (3.4), (3.9) can be written as

$$
\left(\frac{\partial H_{o, n}}{\partial \omega} \frac{\partial H_{o, n}^{*}}{\partial \omega}\right)_{n \omega_{o}}=\left(\frac{2 Q_{n}}{n \omega_{o}}\right)^{2}
$$

(3.10) will be utilized in analysis of the lock range of non-harmonic oscillators.

\subsection{Harmonic Oscillators with Multiple Injections}

Consider the LC oscillator in Fig. 3.3 where $I_{i n j 1}$ and $I_{i n j 2}$ represent the two currents injected at two different nodes of the oscillator.

The injection-locked oscillator in the vicinity of the lock state is represented by the block diagram shown in Fig. 3.3 with $g_{m 1}$ and $g_{m 2}$ the transconductance of M1 and M2, respectively. Write KCL at the output nodes of the transconductors

$$
\begin{aligned}
I_{o 2} & =\left[\left(I_{o 1}-I_{i n j 1}\right) Z_{L 1}\right] g_{m 2} \\
I_{o 1} & =-\left[\left(I_{o 2}-I_{i n j 2}\right) Z_{L 2}\right] g_{m 1}
\end{aligned}
$$

where $Z_{L 1}=s L_{1} \| \frac{1}{s C_{1}}$ and $Z_{L 2}=s L_{2} \| \frac{1}{s C_{2}}$. Let $H_{o 1}=g_{m 2} Z_{L 1}$ and $H_{o 2}=g_{m 1} Z_{L 2}$, (3.11) can be written as 


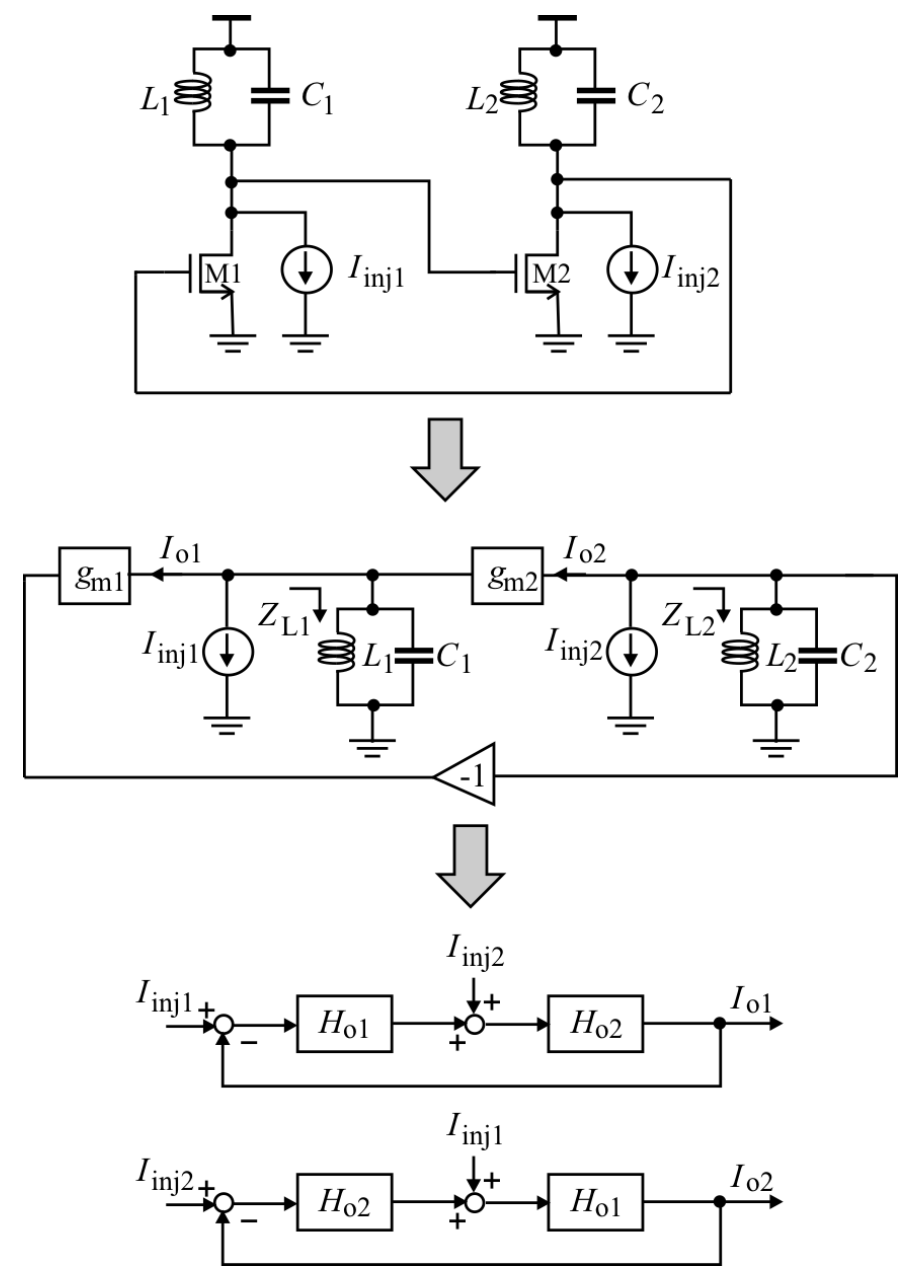

Figure 3.3: Injection-locked harmonic oscillators with multiple injections.

$$
\begin{aligned}
& I_{o 1}=\left(\frac{H_{o 1} H_{o 2}}{1+H_{o 1} H_{o 2}}\right) I_{i n j 1}+\left(\frac{H_{o 2}}{1+H_{o 1} H_{o 2}}\right) I_{i n j 2} . \\
& I_{o 2}=\left(\frac{H_{o 1} H_{o 2}}{1+H_{o 1} H_{o 2}}\right) I_{i n j 2}+\left(\frac{H_{o 1}}{1+H_{o 1} H_{o 2}}\right) I_{i n j 1} .
\end{aligned}
$$

It is evident from (3.12) that the injection-locked oscillator can be represented by the linear control system shown in Fig. 3.3. Eq.(3.11) can also be written as 


$$
\begin{aligned}
& I_{o 2}=H_{o 1} I_{o 1}-H_{o 1} I_{i n j 1}, \\
& I_{o 1}=-H_{o 2} I_{o 2}+H_{o 2} I_{i n j 2} .
\end{aligned}
$$

When the injection signals are absent, we have $I_{o 1}=-H_{o 2} I_{o 2}$ and $I_{o 2}=H_{o 1} I_{o 1}$. Since $I_{o 1}$ and $I_{o 2}$ are out of phase with the same magnitude, we conclude

$$
\begin{aligned}
& H_{o 1}=e^{j \pi} \\
& H_{o 2}=-e^{j \pi} .
\end{aligned}
$$

The results given in (3.14) are important as they will be used to quantify the contribution of the double injections to the lock range of the non-harmonic oscillators.

\subsection{Non-Harmonic Oscillators with Multiple Injections}

Let us extend the control system presentation of harmonic oscillators with multiple injections presented earlier to non-harmonic oscillators with multiple injections. Consider a non-harmonic oscillator with injections $I_{i n j 1}$ and $I_{i n j 2}$. In the vicinity of the lock state, the injection-locked oscillator is represented by the block diagram shown in Fig. 3.4.

$I_{i n j 1}(t)$ affects the oscillator through $I_{i n j 1,1}$ at $\omega_{i n j}, I_{i n j 1,3}$ at $3 \omega_{i n j} \ldots$ and $I_{i n j 2}(t)$ affects the oscillator through $I_{i n j 2,1}$ at $\omega_{i n j}, I_{i n j 2,3}$ at $3 \omega_{i n j}, \ldots$ The output of the non-harmonic oscillator in the lock state is obtained from

$$
\begin{aligned}
I_{o} & =\left[\frac{H_{o 1} I_{i n j 1,1}}{1+H_{o 1}}+\frac{H_{o 1} I_{i n j 2,1}}{H_{o 1,1}\left(1+H_{o 1}\right)}\right] \delta\left(\omega-\omega_{i n j}\right) \\
& +\left[\frac{H_{o 3} I_{i n j 1,3}}{1+H_{o 3}}+\frac{H_{o 3} I_{i n j 2,3}}{H_{o 1,3}\left(1+H_{o 3}\right)}\right] \delta\left(\omega-3 \omega_{i n j}\right) \\
& +\ldots
\end{aligned}
$$




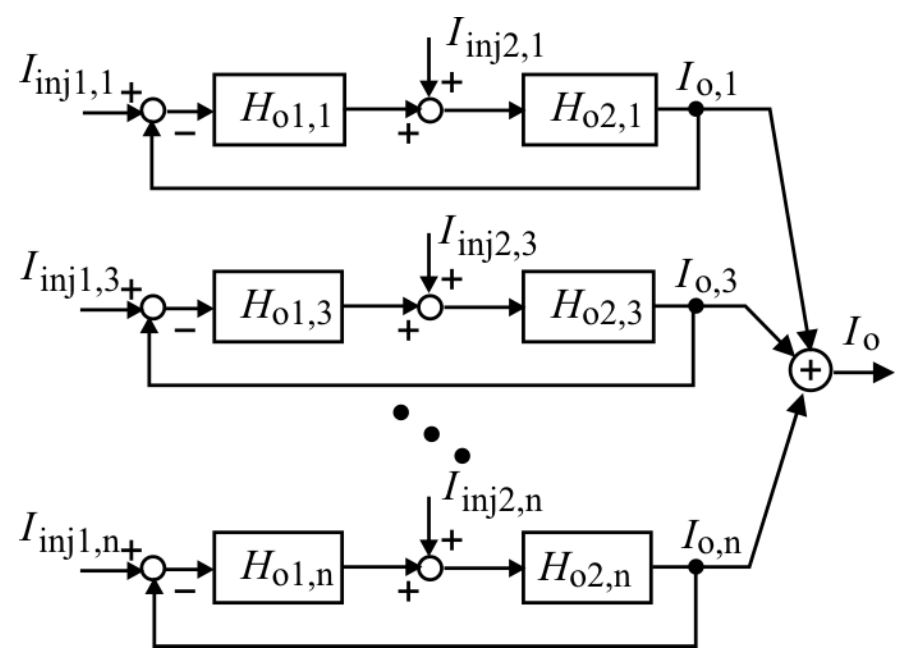

Figure 3.4: Representation of injection-locked non-harmonic oscillators with multiple singletone injections.

where $H_{o 1}=H_{o 1,1} H_{o 2,1}$ and $H_{o 3}=H_{o 1,3} H_{o 2,3}$ are the loop gain of the first-order and 3rdorder harmonic oscillators, respectively.

\subsection{Lock Range of Non-Harmonic Oscillators with Mul- tiple Single-Tone Injections}

Assume that single-tone injection signals $I_{i n j 1}(t)$ and $I_{i n j 2}(t)$ of the same frequency $\omega_{i n j}$ cause the frequency of a non-harmonic oscillator to shift from its natural frequency $\omega_{o}$ to $\omega_{i n j}=\omega_{o}+\Delta \omega$ in the lock state with $\Delta \omega_{o} \ll \omega_{o}$. Using the first-order Taylor series approximation given in $(3.5)$ and noting that $H_{o, n}\left(n \omega_{o}\right)=-1, H_{o, n}\left(n \omega_{o}\right) \gg\left[\frac{\partial H_{o, n}}{\partial \omega}\right]_{n \omega_{o}}(n \Delta \omega)$, and $1+H_{o, n}\left(\omega_{o}+n \Delta \omega\right) \approx 0$, the output of the oscillator in the lock state can be obtained from $(3.15)$ 


$$
\begin{aligned}
I_{o} & \approx\left[\frac{-I_{i n j 1,1}}{\left(\frac{\partial H_{o 1}}{\partial \omega}\right)_{\omega_{o}} \Delta \omega}+\frac{1}{H_{o 1,1}} \frac{-I_{i n j 2,1}}{\left(\frac{\partial H_{o 1}}{\partial \omega}\right)_{\omega_{o}} \Delta \omega}\right] \delta\left(\omega-\omega_{i n j}\right) \\
& +\left[\frac{-I_{i n j 1,3}}{\left(\frac{\partial H_{o 3}}{\partial \omega}\right)_{3 \omega_{o}} 3 \Delta \omega}+\frac{1}{H_{o 1,3}} \frac{-I_{i n j 2,3}}{\left(\frac{\partial H_{o 3}}{\partial \omega}\right)_{3 \omega_{o}} 3 \Delta \omega}\right] \delta\left(\omega-3 \omega_{i n j}\right) \\
& +\ldots
\end{aligned}
$$

It follows from (3.16) that

$$
\begin{aligned}
I_{o}^{*} & \approx\left[\frac{-I_{i n j 1,1}^{*}}{\left(\frac{\partial H_{o 1}^{*}}{\partial \omega}\right)_{\omega_{o}} \Delta \omega}+\frac{1}{H_{o 1,1}^{*}} \frac{-I_{i n j 2,1}^{*}}{\left(\frac{\partial H_{o 1}^{*}}{\partial \omega}\right)_{\omega_{o}} \Delta \omega}\right] \delta\left(\omega-\omega_{i n j}\right) \\
& +\left[\frac{-I_{i n j 1,3}^{*}}{\left(\frac{\partial H_{o 3}^{*}}{\partial \omega}\right)_{3 \omega_{o}}(3 \Delta \omega)}+\frac{1}{H_{o 1,3}^{*}} \frac{-I_{i n j 2,3}^{*}}{\left(\frac{\partial H_{o 3}^{*}}{\partial \omega}\right)_{3 \omega_{o}}(3 \Delta \omega)}\right] \delta\left(\omega-3 \omega_{i n j}\right) \\
& +\ldots
\end{aligned}
$$

Because $\delta\left(\omega-\omega_{m}\right) \delta\left(\omega-\omega_{n}\right)=1$ if $m=n$ and 0 otherwise, we have

$$
\begin{aligned}
I_{o 1} I_{o 1}^{*} \approx & {\left[\frac{\left|I_{i n j 1,1}\right|^{2}}{\left(\frac{\partial H_{o 1}}{\partial \omega} \frac{\partial H_{o 1}^{*}}{\partial \omega}\right)_{\omega_{o}}(\Delta \omega)^{2}}+\frac{1}{\left|H_{o 1,1}\right|^{2}} \frac{\left|I_{i n j 2,1}\right|^{2}}{\left(\frac{\partial H_{o 1}}{\partial \omega} \frac{\partial H_{o 1}^{*}}{\partial \omega}\right)_{\omega_{o}}(\Delta \omega)^{2}}\right.} \\
& \left.+\frac{I_{i n j 1,1} I_{i n j 2,1}^{*}}{H_{o 1,1}^{*}\left(\frac{\partial H_{o 1}}{\partial \omega} \frac{\partial H_{o 1}^{*}}{\partial \omega}\right)_{\omega_{o}}(\Delta \omega)^{2}}+\frac{1}{H_{o 1,1}} \frac{I_{i n j 1,1}^{*} I_{i n j 2,1}}{\left(\frac{\partial H_{o 1}}{\partial \omega} \frac{\partial H_{o 1}^{*}}{\partial \omega}\right)_{\omega_{o}}(\Delta \omega)^{2}}\right] \delta\left(\omega-\omega_{i n j}\right) \\
& +\left[\frac{\left|I_{i n j 1,3}\right|^{2}}{\left(\frac{\partial H_{o 3}}{\partial \omega} \frac{\partial H_{o 3}^{*}}{\partial \omega}\right)_{3 \omega_{o}}(3 \Delta \omega)^{2}}+\frac{1}{\left|H_{o 1,3}\right|^{2}} \frac{\left|I_{i n j 2,3}\right|^{2}}{\left(\frac{\partial H_{o 3}}{\partial \omega} \frac{\partial H_{o 3}^{*}}{\partial \omega}\right)_{3 \omega_{o}}(3 \Delta \omega)^{2}}\right. \\
& \left.+\frac{I_{i n j 1,3} I_{i n j 2,3}^{*}}{H_{o 1,3}^{*}\left(\frac{\partial H_{o 3}}{\partial \omega} \frac{\partial H_{o 3}^{*}}{\partial \omega}\right)_{3 \omega_{o}}(3 \Delta \omega)^{2}}+\frac{1}{H_{o 1,3}} \frac{I_{i n j 1,3}^{*} I_{i n j 2,3}}{\left(\frac{\partial H_{o 3}}{\partial \omega} \frac{\partial H_{o 3}^{*}}{\partial \omega}\right)_{3 \omega_{o}}(3 \Delta \omega)^{2}}\right] \delta\left(\omega-3 \omega_{i n j}\right) \\
& +\ldots
\end{aligned}
$$


Utilizing (3.10), we can write (3.18) as

$$
\begin{aligned}
I_{o} I_{o}^{*} & =\left(\frac{\omega_{o}}{2 Q_{1}}\right)^{2} \frac{1}{(\Delta \omega)^{2}}\left[\left|I_{i n j 1,1}\right|^{2}+\frac{\left|I_{i n j 2,1}\right|^{2}}{\left|H_{o 1,1}\right|^{2}}+\frac{I_{i n j 1,1} I_{i n j 2,1}^{*}}{H_{o 1,1}^{*}}+\frac{I_{i n j 1,1}^{*} I_{i n j 2,1}}{H_{o 1,1}}\right] \delta\left(\omega-\omega_{i n j}\right) \\
& +\left(\frac{\omega_{o}}{2 Q_{3}}\right)^{2} \frac{1}{(\Delta \omega)^{2}}\left[\left|I_{i n j 1,3}\right|^{2}+\frac{\left|I_{i n j 2,3}\right|^{2}}{\left|H_{o 1,3}\right|^{2}}+\frac{I_{i n j 1,3} I_{i n j 2,3}^{*}}{H_{o 1,3}^{*}}+\frac{I_{i n j 1,3}^{*} I_{i n j 2,3}}{H_{o 1,3}}\right] \delta\left(\omega-3 \omega_{i n j}\right) \\
& +\ldots
\end{aligned}
$$

Matching the terms in (3.19) that have the same frequency yields

$$
\begin{aligned}
I_{o, 1}^{2} & =\left(\frac{\omega_{o}}{2 Q_{1}}\right)^{2} \frac{1}{(\Delta \omega)^{2}}\left[\left|I_{i n j 1,1}\right|^{2}+\frac{\left|I_{i n j 2,1}\right|^{2}}{\left|H_{o 1,1}\right|^{2}}+\frac{I_{i n j 1,1} I_{i n j 2,1}^{*}}{H_{o 1,1}^{*}}+\frac{I_{i n j 1,1}^{*} I_{i n j 2,1}}{H_{o 1,1}}\right] \\
I_{o, 3}^{2} & =\left(\frac{\omega_{o}}{2 Q_{3}}\right)^{2} \frac{1}{(\Delta \omega)^{2}}\left[\left|I_{i n j 1,3}\right|^{2}+\frac{\left|I_{i n j 2,3}\right|^{2}}{\left|H_{o 1,3}\right|^{2}}+\frac{I_{i n j 1,3} I_{i n j 2,3}^{*}}{H_{o 1,3}^{*}}+\frac{I_{i n j 1,3}^{*} I_{i n j 2,3}}{H_{o 1,3}}\right] \\
& \ldots
\end{aligned}
$$

Let $I_{i n j 1}(t)$ and $I_{i n j 2}(t)$ have the same amplitude $I_{i n j}$ but different phases

$$
\begin{gathered}
I_{i n j 1}(t)=I_{i n j} e^{j\left(\omega_{i n j} t+\phi_{1}\right)} \\
I_{i n j 2}(t)=I_{i n j} e^{j\left(\omega_{i n j} t+\phi_{2}\right)} .
\end{gathered}
$$

Also, let the magnitude of the injection signals of first-order, 3rd-order, and $n$ th-order harmonic oscillators due to $I_{i n j 1}(t)$ be denoted by $I_{i n j, 1}, I_{i n j, 3}, \ldots, I_{i n j, n}$, respectively. Similar notations apply to $I_{i n j 2}(t)$ as well. Further, let $\Delta \phi=\phi_{1}-\phi_{2}$ be the phase difference between $I_{i n j 1}(t)$ and $I_{i n j 2}(t)$. Since (3.14) holds for harmonic oscillators with double injections, it will also hold for $n$ th-order harmonic oscillator

$$
\begin{aligned}
& H_{o 1, n}=e^{j \pi} \\
& H_{o 2, n}=-e^{j \pi}
\end{aligned}
$$


$n=1,3,5, \ldots(3.20)$ becomes

$$
\begin{aligned}
I_{o, 1}^{2} & =\left(\frac{\omega_{o}}{2 Q_{1}}\right)^{2} \frac{I_{i n j, 1}^{2}}{(\Delta \omega)^{2}}\left[2+e^{j(\pi+\Delta \phi)}+e^{-j(\pi+\Delta \phi)}\right] \\
I_{o, 3}^{2} & =\left(\frac{\omega_{o}}{2 Q_{3}}\right)^{2} \frac{I_{i n j, 3}^{2}}{(\Delta \omega)^{2}}\left[2+e^{j(\pi+\Delta \phi)}+e^{-j(\pi+\Delta \phi)}\right] \\
& \ldots
\end{aligned}
$$

Since $I_{o, 1}, I_{o, 3}, \ldots$ are the magnitude of the fundamental and harmonics of $I_{o}(t)$, respectively. As

$$
I_{o}^{2}=I_{o, 1}^{2}+I_{o, 3}^{2}+I_{o, 5}^{2}+\ldots
$$

is the output power of the oscillator. The summation of the expressions in (3.24) yields

$$
\begin{aligned}
(\Delta \omega)^{2}= & {\left[\left(\frac{\omega_{o}}{2 Q_{1}}\right)^{2} \frac{I_{i n j, 1}^{2}}{I_{o}^{2}}+\left(\frac{\omega_{o}}{2 Q_{3}}\right)^{2} \frac{I_{i n j, 3}^{2}}{I_{o}^{2}}+\ldots\right] } \\
& {\left[2+e^{j(\pi+\Delta \phi)}+e^{-j(\pi+\Delta \phi)}\right] . }
\end{aligned}
$$

Define

$$
\left|\Delta \omega_{n}\right|=\left(\frac{\omega_{o}}{2 Q_{n}}\right) \frac{I_{i n j, n}}{I_{o}} .
$$

The denominator of (3.27) is the total output current of the oscillator. (3.27) thus quantifies the contribution of $n$ th-order harmonic oscillator $I_{i n j, n}$ to the overall lock range of the nonharmonic oscillator. (3.26) can be written as

$$
|\Delta \omega|=\alpha_{d} \sqrt{\sum_{n=1,3, \ldots}^{\infty}\left(\Delta \omega_{n}\right)^{2}}
$$


where

$$
\alpha_{d}=\sqrt{2+e^{j(\pi+\Delta \phi)}+e^{-j(\pi+\Delta \phi)}} .
$$

Since

$$
\left|\Delta \omega_{s}\right|=\sqrt{\sum_{n=1,3, \ldots}^{\infty}\left(\Delta \omega_{n}\right)^{2}}
$$

is the lock range of the non-harmonic oscillator with a single injection, the lock range of the oscillator with double injections given by (3.28) can be written as

$$
\left|\Delta \omega_{d}\right|=\alpha_{d}\left|\Delta \omega_{s}\right|
$$

The preceding developments are summarised as:

1. (3.25) quantifies the output power of the non-harmonic oscillator (assume $1 \Omega$ load). This can be best explained using Fourier series representation of periodic functions. Consider a periodic function $i(t)$ and represent it with the Fourier series $i(t)=\sum_{n=1,3, \ldots}^{\infty} I_{n} \cos (n \omega t)$ where $\omega=2 \pi / T$ and $T$ is the period of $i(t)$. The average power of $i(t)$, denoted $P_{\text {avg }}$, is obtained from [104]

$$
P_{a v g}=\sqrt{\frac{1}{T} \int_{0}^{T} i^{2}(t) d t}=\sqrt{\sum_{n=1,3, \ldots}^{\infty}\left(\frac{I_{n}}{\sqrt{2}}\right)^{2}}
$$

Clearly the average power of $i(t)$ is the sum of that of each harmonic of $i(t)$. Since $i(t)$ contains an infinite number of frequency components, its power is distributed across the entire frequency spectrum. The actual profile of the distribution of the power of $i(t)$ is determined by the characteristics of $i(t)$. For example, if $i(t)$ is a sinusoid of 
frequency $\omega_{o}$, its power will only be concentrated at $\omega_{o}$. If $i(t)$ is a square wave, its power will mainly be concentrated at the fundamental and low-order harmonics.

2. $\Delta \omega_{n}$ is the lock range of $n$ th-harmonic oscillator with a single injection. (3.30) thus quantifies the relation between the lock range of the harmonic oscillators and that of the non-harmonic oscillators. For example, if the harmonic oscillator is injectionlocked to an external signal, only fundamental frequency will be taken into account. And therefore (3.30) is simplified to (3.33)

$$
\left|\Delta \omega_{s}\right|=\left(\frac{\omega_{o}}{2 Q}\right)\left|\frac{I_{i n j}}{I_{o}}\right|
$$

same as (2.5).

3. $\alpha_{d}$ quantifies the effect of the phase relation between the two injection signals on the lock range of the non-harmonic oscillator. Table 3.1 tabulates $\alpha_{d}$ for several special cases. It is evident that the lock range of non-harmonic oscillators with multiple single-tone injections will be large than that with a single-tone injection, if the phases of these injection signals are properly chosen. This finding can also be applied to injection-locked harmonic oscillators.

Table 3.1: Lock Range Coefficient $\alpha_{d}$

\begin{tabular}{l|l}
\hline Phase difference $(\Delta \phi)$ & Lock range coeff. $\left(\alpha_{d}\right)$ \\
\hline$\Delta \phi=0$ & $\alpha_{d}=0$ \\
$\Delta \phi=\pi / 4$ & $\alpha_{d}=\sqrt{2\left(1-\cos \frac{\pi}{4}\right)}$ \\
$\Delta \phi=\pi / 2$ & $\alpha_{d}=\sqrt{2}$ \\
$\Delta \phi=3 \pi / 4$ & $\alpha_{d}=\sqrt{2\left(1+\cos \frac{\pi}{4}\right)}$ \\
$\Delta \phi=\pi$ & $\alpha_{d}=2$ \\
\hline
\end{tabular}




\subsection{Simulations}

Relaxation oscillators are widely encountered in passive wireless microsystems owing to their low sensitivity to supply voltage fluctuation as the charging and discharging currents are well defined by the current references, and their low sensitivity to temperature variation as its performance is largely determined by passive components and digital components. The harsh nonlinear elements of relaxation oscillators are comparators and the switching instants of the comparator are known a prior in the steady state, the comparators, therefore, can be modelled as periodically-operated switches that connect the output of the comparators to either $V_{D D}$ or ground. If no other nonlinearities are considered, relaxation oscillators can be modelled as periodically time-varying linear circuits. If nonlinearities other than comparators are also considered, relaxation oscillators are periodically time-varying nonlinear circuits. An injection-locked dual-comparator relaxation oscillator shown in Fig. 3.5, operating in the sub-threshold mode with $13 \mathrm{MHz}$ frequency for power saving, to verify all the findings presented earlier and later. The oscillator is designed in IBM $130 \mathrm{~nm}$ 1.2V CMOS technology and analyzed using Cadence Spectre.

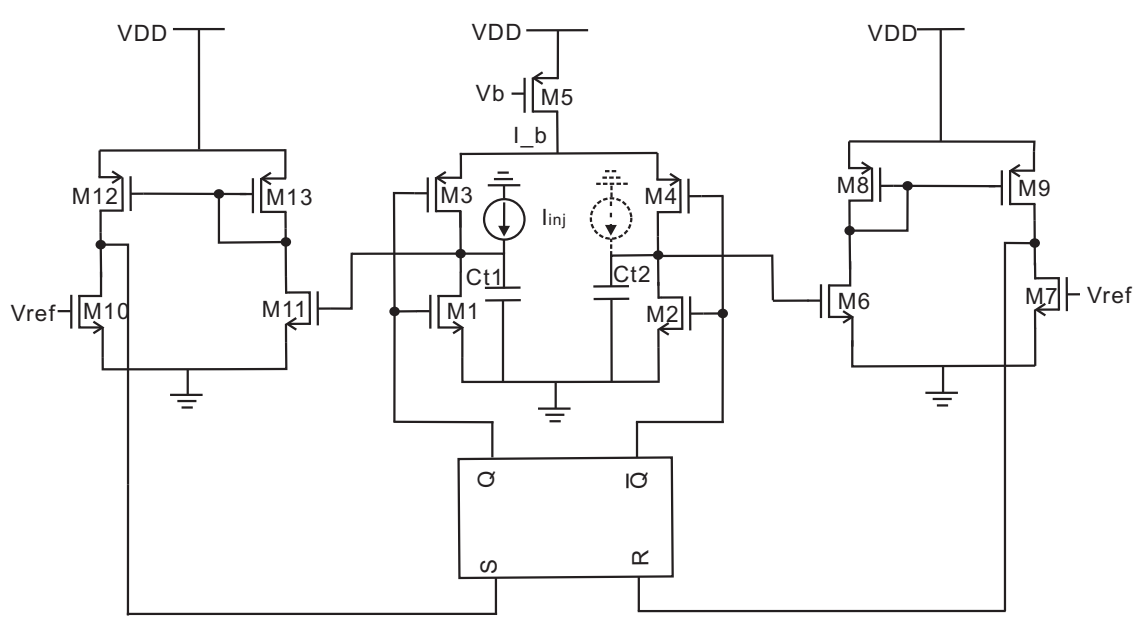

Figure 3.5: Schematic of relaxation oscillator with injection-locking. Circuit parameters: $W_{1,2,6,7,10,11}=0.5 \mu \mathrm{m}, W_{3,4}=1.2 \mu \mathrm{m}, W_{8,9,12,13}=1.25 \mu \mathrm{m}, W_{5}=2 \mu \mathrm{m}$, Supply voltage $V_{D D}=$ $0.9 \mathrm{~V}, V_{\text {ref }}=0.31 \mathrm{~V}, I_{b}=253 \mathrm{nA}, V_{b}=0.62 \mathrm{~V}$ 
Fig. 3.6 shows free-running frequency of the relaxation oscillator without disturbance of the injecting signal.

The spectrum of the oscillator is used to determine whether the oscillator is locked or not. The locking of an oscillator could be fast beat ( $\omega_{i n j}$ is far away from $\omega_{o}$ and outside the lock range), quasi lock ( $\omega_{i n j}$ is very close to $\omega_{o}$ but outside the lock range), and lock ( $\omega_{i n j}$ is in the lock range) [53]. The spectrum of the oscillator in fast beat consists of a train of tones separated with a large frequency spacing while that of the oscillator in quasi lock is made of a train of tones separated with a small frequency spacing. When the oscillator is locked to the injection signal, only tones at the frequencies of the injection signal will exist $[105,106,107,108]$. One therefore can determine whether a lock state is established or not by examining the spectrum of the oscillator. Figs.3.7, 3.8, and 3.9 show the spectrum of the relaxation oscillator (taken at the output of the comparators) in fast beat, quasi-lock, and lock states with a square-wave injection of injection ratio 0.1. Injection ratio is defined as the ratio of the injection current $I_{i n j}$ to the tail current $I_{b}$.

Double injection is obtained by injecting a current source from the drain of M1 to the drain of M2. In this case, the two injection currents have the same frequency and amplitude but a 180 degree phase difference. Fig. 3.10 presents the dependence of the lock range on the strength of the injection signal with a sinusoidal injection and double sinusoidal injections whose phase difference is 180 phase. It is seen that increasing injection signal strength yields a larger lock range. Also observed is that for the same injection strength, the lock range of the oscillator with double sinusoidal injections is twice that with a single sinusoidal injection. This agrees well with the theoretical findings presented in Table 3.1.

\subsection{Lock Range of Non-Harmonic Oscillators with Mul- tiple Multi-Tone Injections}

If injection signals are multi-tones, the output of the oscillator can be obtained by considering the effect of each of the harmonic components of the injection signals. Consider a non-harmonic oscillator with two multi-tone injection signals $I_{i n j 1}(t)$ and $I_{i n j 2}(t)$, such as 

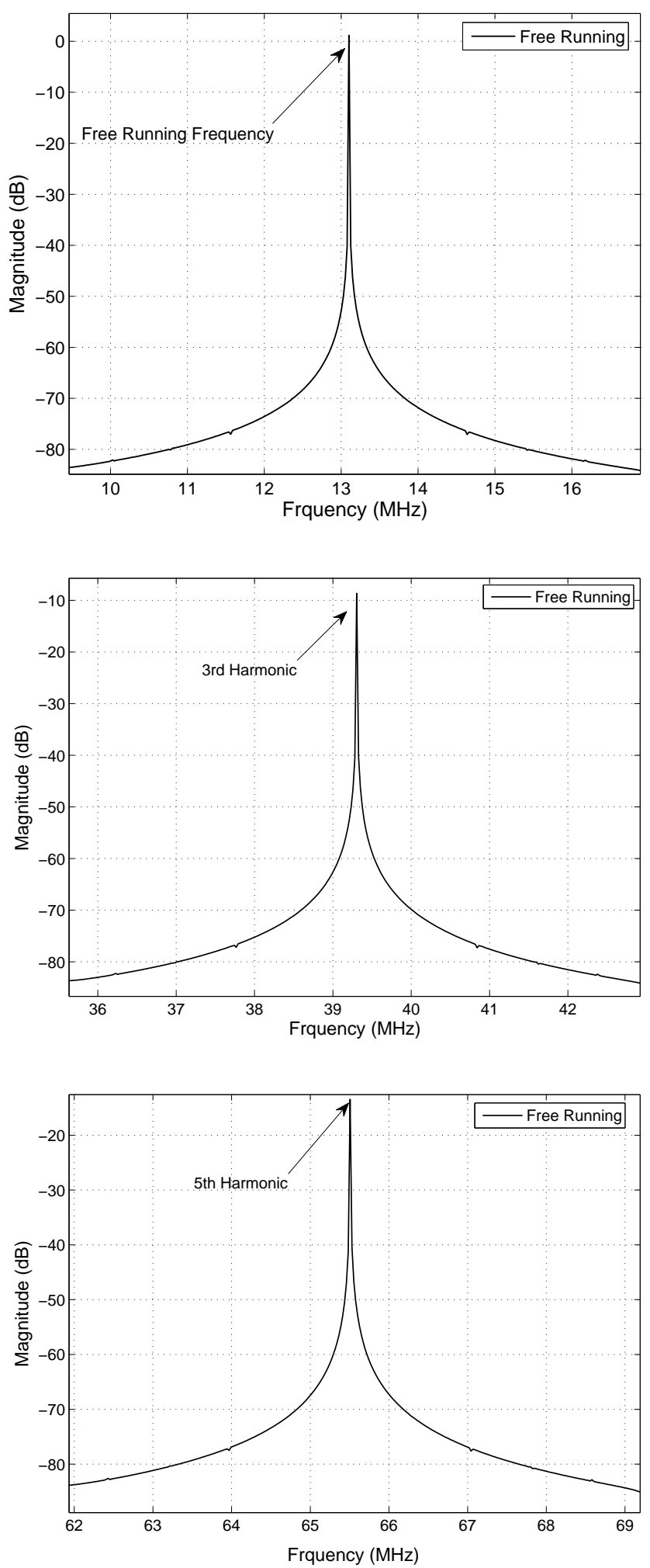

Figure 3.6: Simulated spectrum of relaxation oscillator without injection. Top - Fundamental. Middle : 3rd harmonic. Bottom : 5th harmonic. 

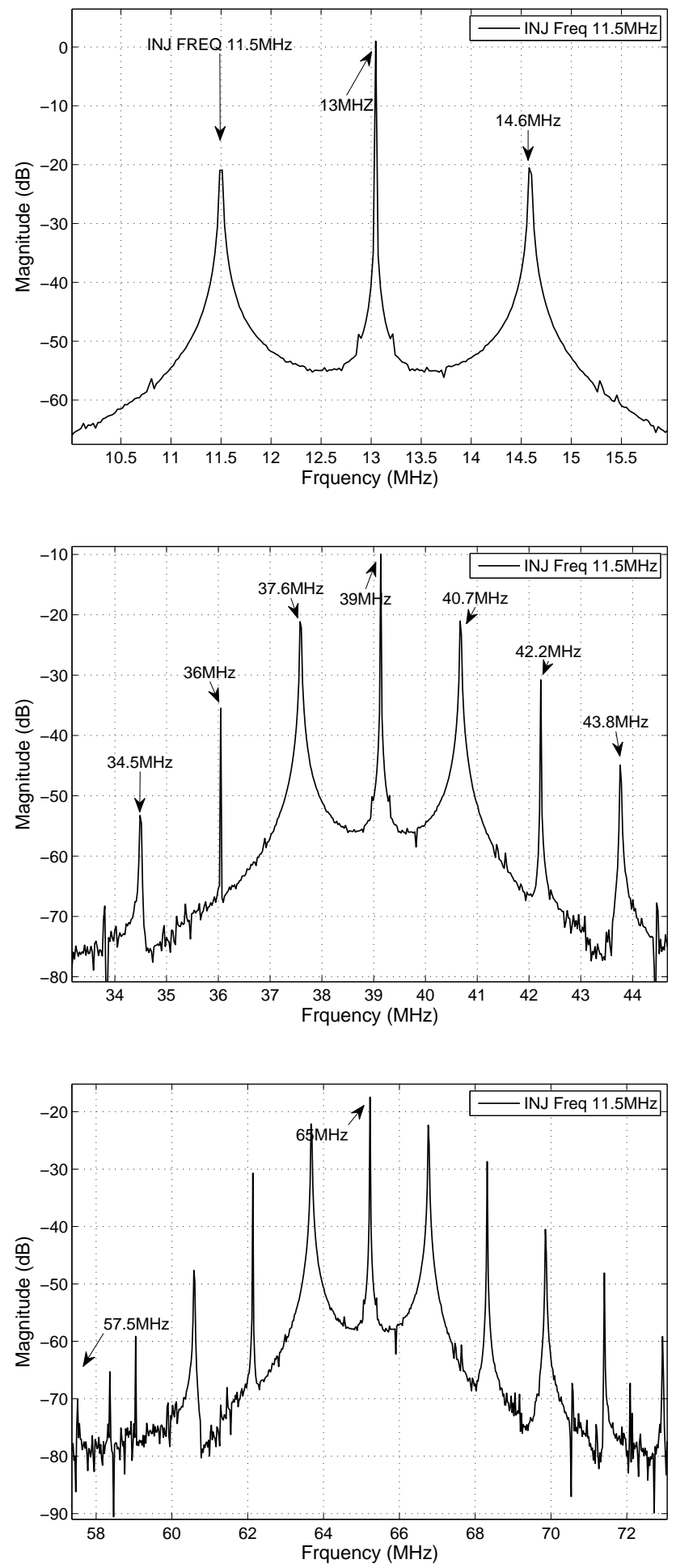

Figure 3.7: Simulated spectrum of relaxation oscillator in fast beat. Top - Fundamental. Middle : 3rd harmonic. Bottom : 5th harmonic. 

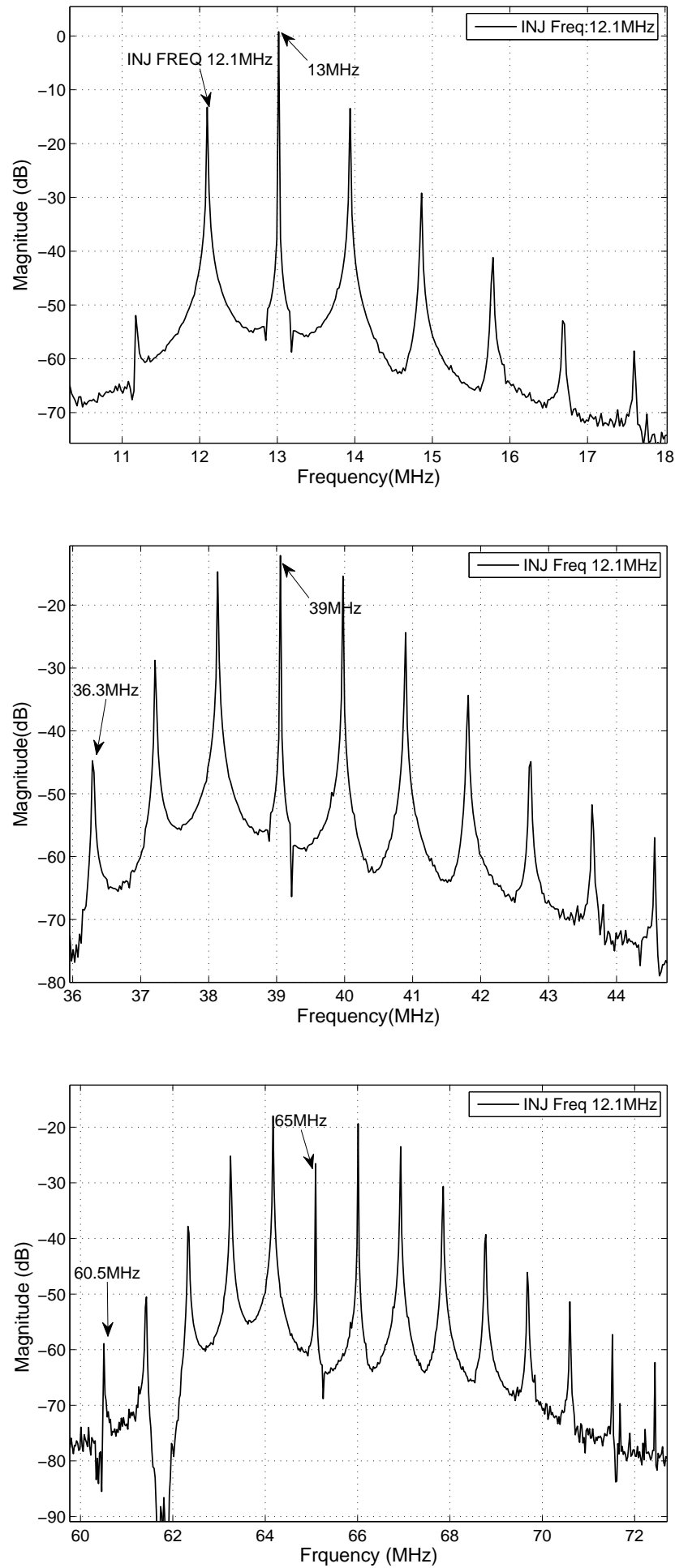

Figure 3.8: Simulated spectrum of relaxation oscillator in quasi-lock. Top - Fundamental. Middle : 3rd harmonic. Bottom : 5th harmonic. 

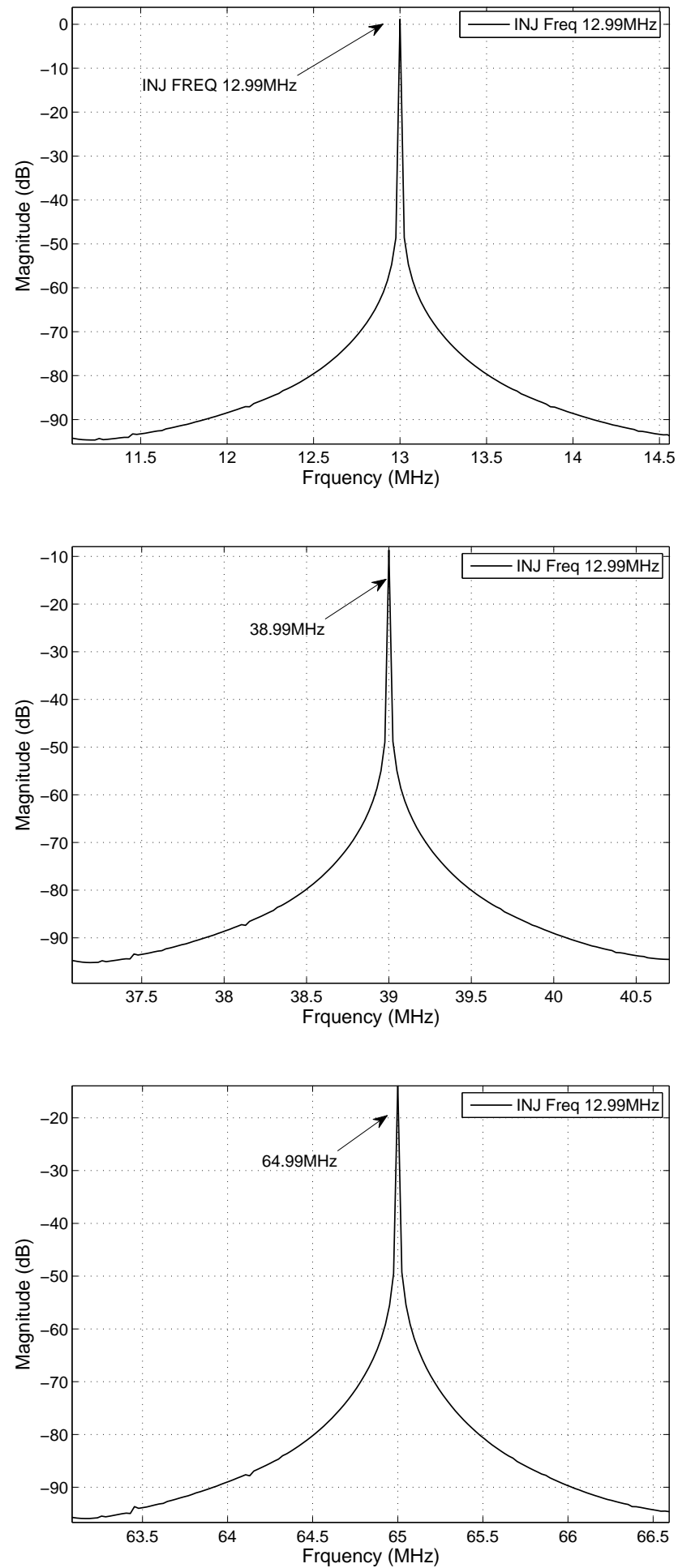

Figure 3.9: Simulated spectrum of relaxation oscillator in lock state. Top - Fundamental. Middle : 3rd harmonic. Bottom : 5th harmonic. 


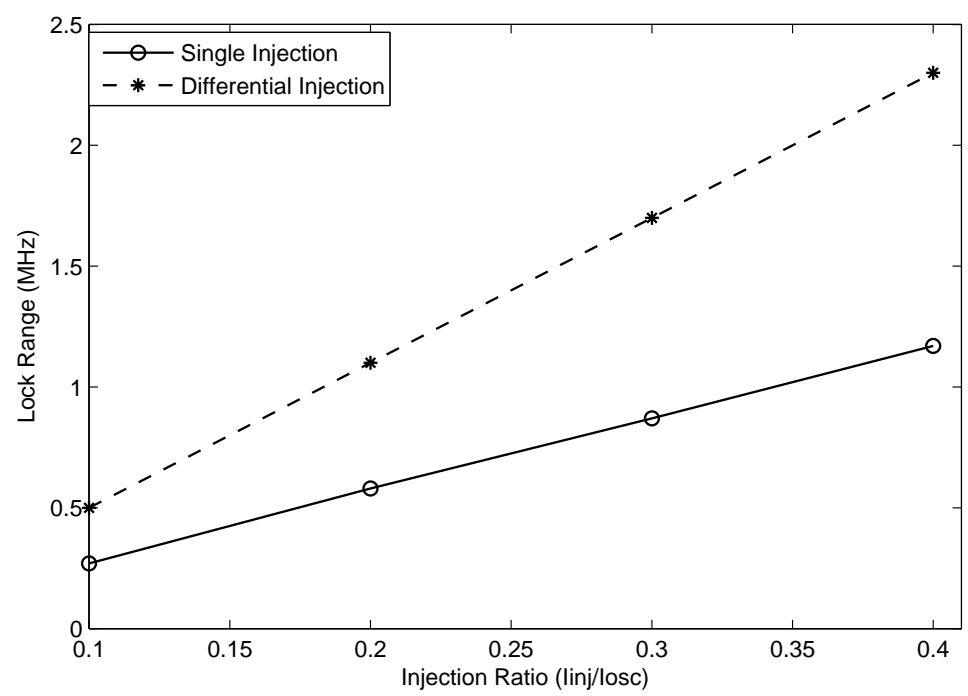

Figure 3.10: Simulated dependence of the lock range of relaxation oscillator with a sinusoidal injection and double sinusoidal injections whose phase difference is 180 degree on injection strength.

square waves. $I_{i n j 1}(t)$ contains singe-tone frequency components $I_{i n j 1,1}, I_{i n j 1,3}, \ldots I_{i n j, n}$ at $\omega_{i n j}, 3 \omega_{i n j}, \ldots, n \omega_{i n j}$, respectively. The same for $I_{i n j 2}(t)$ as well. The effect of $I_{i n j 1,1}$ can be analyzed in a similar way as that for injection-locked non-harmonic oscillators with a singletone injection presented earlier. Specifically, assume that the injection signals of first-order, 3rd-order, $\ldots, n$ th-order harmonic oscillators due to $I_{i n j 1,1}$ are $I_{i n j 1,11}, I_{i n j 1,31}, \ldots, I_{i n j 1, n 1}$, respectively. Similar notations are used for $I_{i n j 2}$, as shown graphically in Fig. 3.11.

The input of first-order harmonic oscillator due to $I_{i n j 1}(t)$ is obtained by summing up $I_{i n j 1,11}$, $I_{i n j 1,13}, \ldots$, and $I_{i n j 1,1 n}$. It is evident that

$$
\begin{aligned}
I_{o} & \approx\left[\frac{-\left(I_{i n j 1,11}+I_{i n j 1,13}+\ldots\right)}{\left(\frac{\partial H_{o 1}}{\partial \omega}\right)_{\omega_{o}} \Delta \omega}+\frac{1}{H_{o 1,1}} \frac{-\left(I_{i n j 2,11}+I_{i n j 2,13}+\ldots\right)}{\left(\frac{\partial H_{o 1}}{\partial \omega}\right)_{\omega_{o}} \Delta \omega}\right] \delta\left(\omega-\omega_{i n j}\right) \\
& +\left[\frac{-\left(I_{i n j 1,31}+I_{i n j 1,33}+\ldots\right)}{\left(\frac{\partial H_{o 3}}{\partial \omega}\right)_{3 \omega_{o}} 3 \Delta \omega}+\frac{1}{H_{o 1,3}} \frac{-\left(I_{i n j 2,31}+I_{i n j 2,33}+\ldots\right)}{\left(\frac{\partial H_{o 3}}{\partial \omega}\right)_{3 \omega_{o}} 3 \Delta \omega}\right] \delta\left(\omega-3 \omega_{i n j}\right) \\
& +\ldots
\end{aligned}
$$




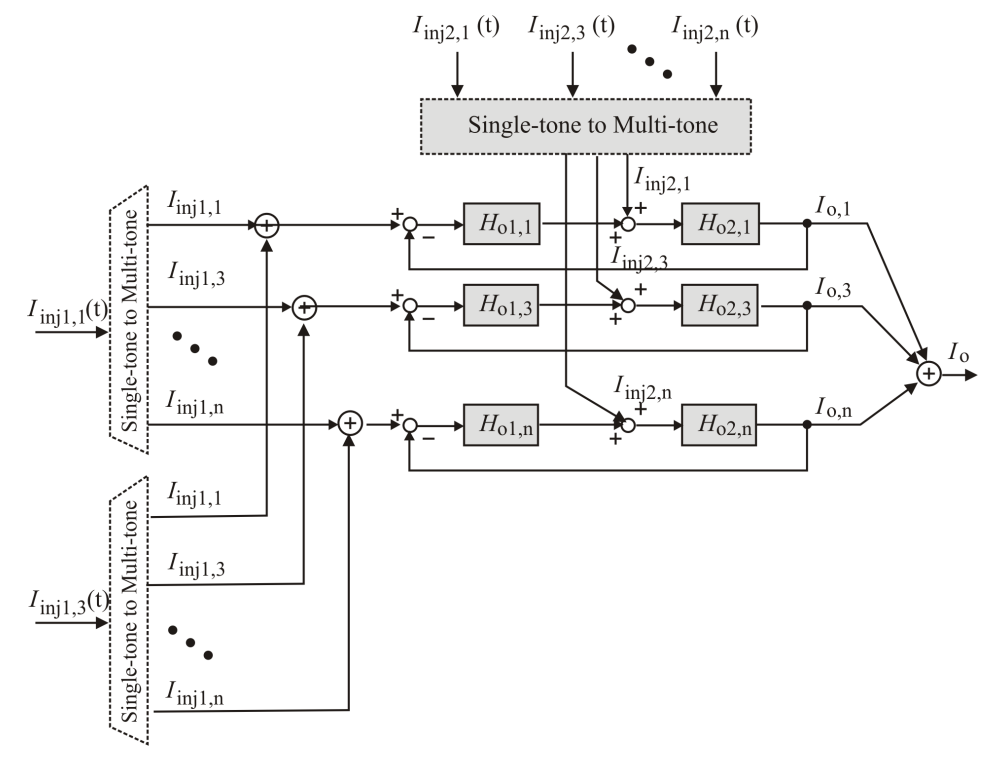

Figure 3.11: Representation of injection-locked non-harmonic oscillators with multiple multitone injections.

It follows from (3.34) that

$$
\begin{aligned}
I_{o}^{*} & \approx\left[\frac{-\left(I_{i n j 1,11}^{*}+I_{i n j 1,13}^{*}+\ldots\right)}{\left(\frac{\partial H_{o 1}^{*}}{\partial \omega}\right)_{\omega_{o}} \Delta \omega}+\frac{1}{H_{o 1,1}^{*}} \frac{-\left(I_{i n j 2,11}^{*}+I_{i n j 2,13}^{*}+\ldots\right)}{\left(\frac{\partial H_{o 1}^{*}}{\partial \omega}\right)_{\omega_{o}} \Delta \omega}\right] \delta\left(\omega-\omega_{i n j}\right) \\
& +\left[\frac{-\left(I_{i n j 1,31}^{*}+I_{i n j 1,33}^{*}+\ldots\right)}{\left(\frac{\partial H_{o 3}^{*}}{\partial \omega}\right)_{3 \omega_{o}} 3 \Delta \omega}+\frac{1}{H_{o 1,3}^{*}} \frac{-\left(I_{i n j 2,31}^{*}+I_{i n j 2,33}^{*}+\ldots\right)}{\left(\frac{\partial H_{o 3}^{*}}{\partial \omega}\right)_{3 \omega_{o}} 3 \Delta \omega}\right] \delta\left(\omega-3 \omega_{i n j}\right) \\
& +\ldots
\end{aligned}
$$


As a result,

$$
\begin{aligned}
I_{o} I_{o}^{*} \approx & {\left[\frac{\left(I_{i n j 1,11}+I_{i n j 1,13}+\ldots\right)\left(I_{i n j 1,11}^{*}+I_{i n j 1,13}^{*}+\ldots\right)}{\left(\frac{\partial H_{o 1}}{\partial \omega} \frac{\partial H_{o 1}^{*}}{\partial \omega}\right)_{\omega_{o}}(\Delta \omega)^{2}}\right.} \\
+ & \frac{\left(I_{i n j 1,11}+I_{i n j 1,13}+\ldots\right)\left(I_{i n j 2,11}^{*}+I_{i n j 2,13}^{*}+\ldots\right)}{H_{o 1,1}\left(\frac{\partial H_{o 1}}{\partial \omega} \frac{\partial H_{o 1}^{*}}{\partial \omega}\right)_{\omega_{o}}(\Delta \omega)^{2}} \\
+ & \frac{\left(I_{i n j 1,11}+I_{i n j 1,13}+\ldots\right)\left(I_{i n j 2,11}^{*}+I_{i n j 2,13}^{*}+\ldots\right)}{H_{o 1,1}\left(\frac{\partial H_{o 1}}{\partial \omega} \frac{\partial H_{o 1}^{*}}{\partial \omega}\right)_{\omega_{o}}(\Delta \omega)^{2}} \\
+ & \left.\frac{\left(I_{i n j 2,11}+I_{i n j 2,13}+\ldots\right)\left(I_{i n j 2,11}^{*}+I_{i n j 2,13}^{*}+\ldots\right)}{\left|H_{o 1,1}\right|^{2}\left(\frac{\partial H_{o 1}}{\partial \omega} \frac{\partial H_{o 1}^{*}}{\partial \omega}\right)_{\omega_{o}}(\Delta \omega)^{2}}\right] \delta\left(\omega-w_{i n j}\right) \\
+ & {\left[\frac{\left(I_{i n j 1,31}+I_{i n j 1,33}+\ldots\right)\left(I_{i n j 1,31}^{*}+I_{i n j 1,33}^{*}+\ldots\right)}{\left(\frac{\partial H_{o 3}}{\partial \omega} \frac{\partial H_{o 3}^{*}}{\partial \omega}\right)_{\omega_{o}}(3 \Delta \omega)^{2}}\right.} \\
+ & \frac{\left(I_{i n j 1,31}+I_{i n j 1,33}+\ldots\right)\left(I_{i n j 2,31}^{*}+I_{i n j 2,33}^{*}+\ldots\right)}{H_{o 3,1^{*}}\left(\frac{\partial H_{o 3}}{\partial \omega} \frac{\partial H_{o 3}^{*}}{\partial \omega}\right)_{\omega_{o}}(3 \Delta \omega)^{2}} \\
+ & \frac{\left(I_{i n j 2,31}+I_{i n j 2,33}+\ldots\right)\left(I_{i n j 2,31}^{*}+I_{i n j 2,33}^{*}+\ldots\right)}{\left|H_{o 3,1}\right|^{2}\left(\frac{\partial H_{o 3}}{\partial \omega} \frac{\partial H_{o 3}^{*}}{\partial \omega}\right)_{\omega_{o}}(3 \Delta \omega)^{2}}\left(\omega-3 w_{i n j}\right) \\
+ & \frac{\left(I_{i n j 1,31}+I_{i n j 1,33}+\ldots\right)\left(I_{i n j 2,31}^{*}+I_{i n j 2,33}^{*}+\ldots\right)}{\partial \omega}(3 \Delta \omega)^{2}
\end{aligned}
$$

Matching the components that have the same frequency in (3.36) yields 


$$
\begin{aligned}
I_{o 1}^{2} & =\left(\frac{\omega_{o}}{2 Q_{1}}\right)^{2} \frac{1}{(\Delta \omega)^{2}}\left[\left(I_{i n j 1,11}+I_{i n j 1,13}+\ldots\right)\left(I_{i n j 1,11}^{*}+I_{i n j 1,13}^{*}+\ldots\right)\right. \\
& +\frac{\left(I_{i n j 1,11}+I_{i n j 1,13}+\ldots\right)\left(I_{i n j 2,11}^{*}+I_{i n j 2,13}^{*}+\ldots\right)}{H_{o 1,1}^{*}} \\
& +\frac{\left(I_{i n j 1,11}+I_{i n j 1,13}+\ldots\right)\left(I_{i n j 2,11}^{*}+I_{i n j 2,13}^{*}+\ldots\right)}{H_{o 1,1}} \\
& \left.+\frac{\left(I_{i n j 2,11}+I_{i n j 2,13}+\ldots\right)\left(I_{i n j 2,11}^{*}+I_{i n j 2,13}^{*}+\ldots\right)}{\left|H_{o 1,1}\right|^{2}}\right] \\
& =\left(\frac{\omega_{o}}{2 Q_{1}}\right)^{2} \frac{1}{(\Delta \omega)^{2}} \hat{I}_{o 1}^{2},
\end{aligned}
$$

where $\hat{I}_{o 1}^{2}$ represents the sum of the terms in the bracket in (3.37). Similarly, we have

$$
I_{o n}^{2}=\left(\frac{\omega_{o}}{2 Q_{n}}\right)^{2} \frac{1}{(\Delta \omega)^{2}} \hat{I}_{o n}^{2}, \quad n=1,3,5, \ldots
$$

The summation of the expressions in (3.38) with utilization of (3.25) yields

$$
(\Delta \omega)^{2}=\left(\frac{\omega_{o}}{2 Q_{1}}\right)^{2} \frac{\hat{I}_{o 1}^{2}}{I_{o}^{2}}+\left(\frac{\omega_{o}}{2 Q_{3}}\right)^{2} \frac{\hat{I}_{o 3}^{2}}{I_{o}^{2}}+\ldots
$$

Define

$$
\left(\Delta \omega_{n}\right)^{2}=\left(\frac{\omega_{o}}{2 Q_{1}}\right)^{2} \frac{\hat{I}_{o n}^{2}}{I_{o}^{2}}
$$

we arrive at

$$
|\Delta \omega|=\sqrt{\sum_{n=1,3,5, \ldots}^{\infty}\left(\Delta \omega_{n}\right)^{2}}
$$


Since the square-wave injections are represented by a set of harmonic single-tone injections, (3.20) will also hold. The lock range of non-harmonic oscillators with double square-wave injections is maximized if the phase difference between the injection signals is 180 degree. A direct comparison of (3.20) with (3.37) reveals that $I_{o n}^{2}, n=1,3,5, \ldots$ of the non-harmonic oscillator with multiple multi-tone injections is larger than that of the non-harmonic oscillator with multiple single-tone injections if the phases of these injections are properly chosen. The large lock range is due to the effect of the harmonic tones of the injection signals.

\subsection{Simulations}

Using same relaxation oscillator in Fig. 3.5 to validate the theoretical analysis. Three different injection signals, namely (i) sinusoidal currents (single-tone injection) $I_{i n j}=$ $A \sin \left(\omega_{i n j} t\right)$, (ii) dual-sinusoidal currents (dual-tone injections) $I_{i n j}(t)=I_{1} \sin \left(\omega_{i n j} t\right)+I_{3} \sin \left(3 \omega_{i n j} t\right)$ with $I_{3}=I_{1} / 3$, and (iii) square-wave currents (multi-tone injections) are used to lock the oscillators. Both single injection and double injections are considered. Fig. 3.12 investigates the dependence of the lock range of the relaxation oscillator on the strength of the injection signal with a single square-wave injection and with double square-wave injections whose phase difference is 180 degree. It is seen that the lock range of the oscillator with double square-wave injections is twice that with a single square-wave injection. This observation also agrees well with the theoretical findings presented earlier.

To investigate whether the dependence of the lock of the relaxation oscillator on injection strength is linear or nonlinear over a large range of injection ratio, the lock range of the relaxation oscillator with a differential square-wave injection is obtained over the injection ratio $0.1 \sim 0.5$ and the results are plotted in Fig. 3.13. It is observed that the dependence of the lock range on the injection ratio is approximately linear over the investigated injection ratio range. It is well known that LC-oscillators typically exhibit a nonlinear lock range characteristics when injection is strong [109, 64]. The nonlinear characteristics of the lock range of these oscillators is largely determined by the frequency selectivity or equivalently the 


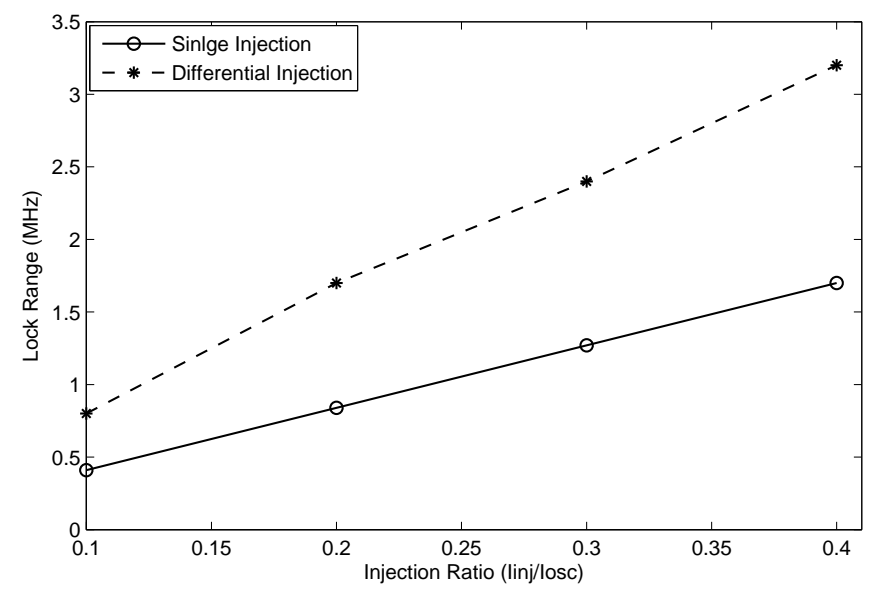

Figure 3.12: Simulated dependence of the lock range of relaxation oscillator with a single square-wave injection and with double square-wave injections whose phase difference is 180 degree on injection strength.

quality factor of the LC tank [110]. It is also known that non-harmonic oscillators typically exhibit a lower quality factor, reflected by their inferior phase noise performance as compared with harmonic oscillators. The low quality factor might contribute to the large lock range subsequently better lock range linearity. This is observed in injection-locked active-inductor LC oscillators, which have a larger lock range as compared with their spiral LC counterparts. The sub-threshold operation of the relaxation oscillator investigated might also contribute to the large lock range and better lock range linearity as the current of MOSFETs is an exponential rather than a second-order function of the over-drive voltage.

Fig. 3.14 compares the lock range of the oscillator with a single sinusoidal injection, a single square-wave injection, and a single dual-tone (two-sinusoids) injection. It is seen that the oscillator with a square-wave injection yields the largest lock range whereas that with a single sinusoidal injection has the smallest lock range. These results echo the theoretical findings given earlier well.

Fig. 3.15 plots the dependence of the lock range of the oscillator with double squarewave injections on the phase difference of the injection signals. It is evident that the lock range increases monotonically with the phase difference and reaches its maximum when the phase difference is 180 degree. This agrees well with the theoretical results derived earlier. 


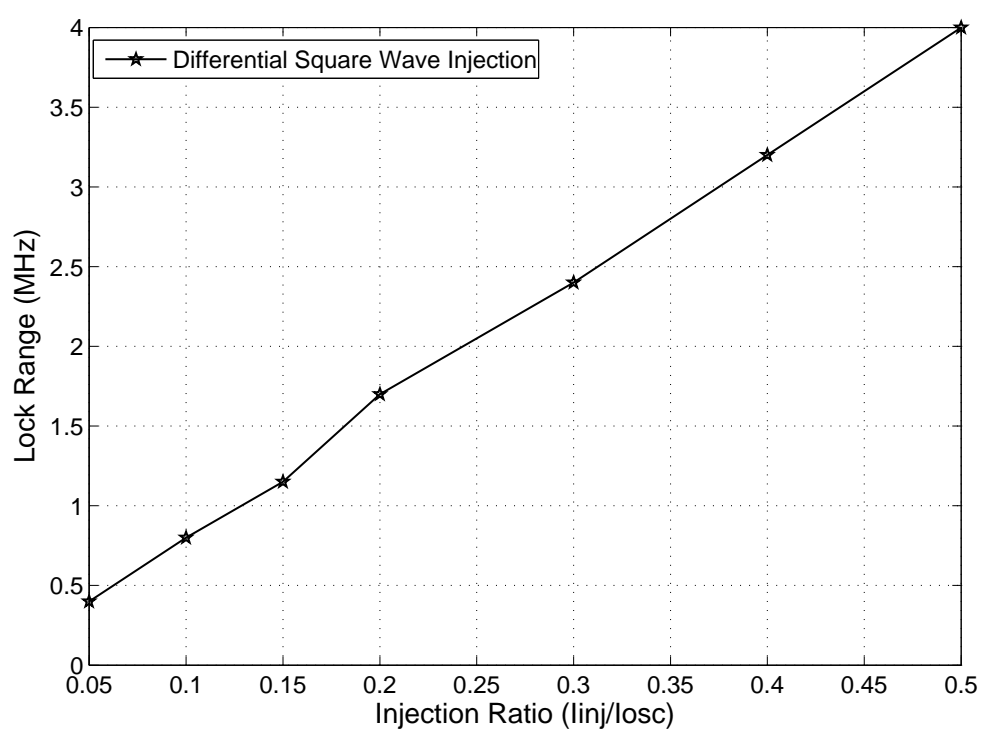

Figure 3.13: Simulated dependence of the lock range of relaxation oscillator with differential square-wave injection on injection strength.

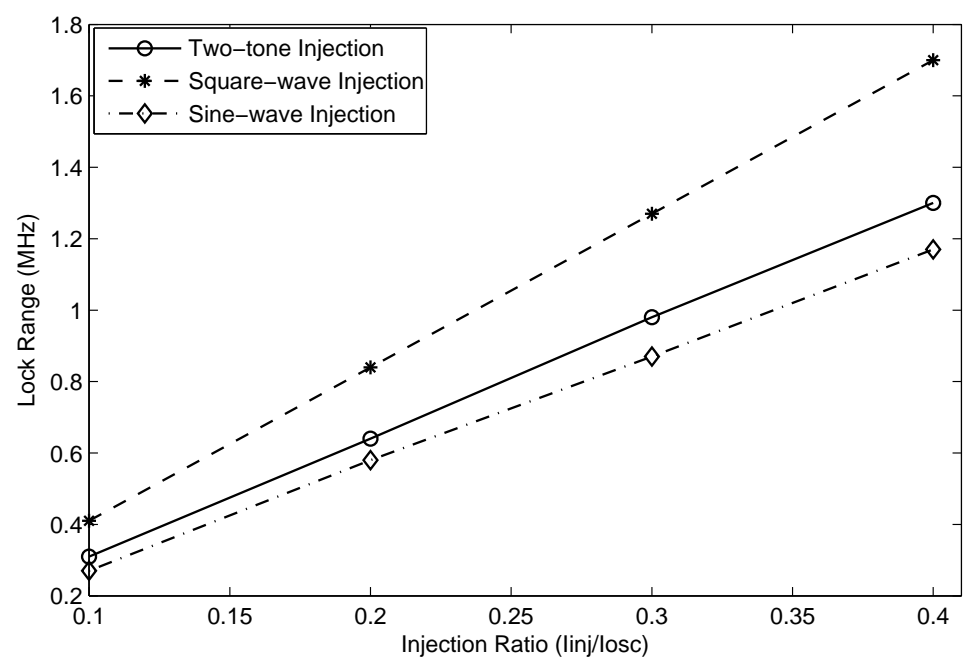

Figure 3.14: Simulated dependence of the lock range of relaxation oscillator with a single sinusoidal injection, a single square-wave injection, and a single dual-tone (two-sinusoids) injection on injection strength.

Fig. 3.16 compares the lock range of the relaxation oscillator with a two-tone sinusoidal injection and double two-tone injections whose phase difference is 180 degree. It is seen that for the same injection strength, the lock range of the oscillator with double two- 


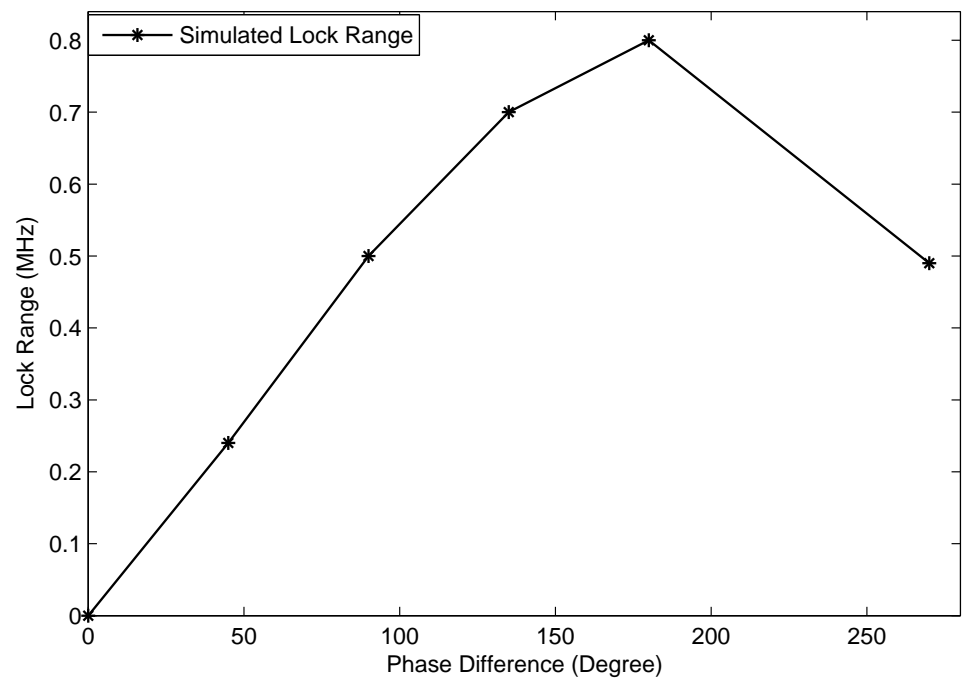

Figure 3.15: Simulated dependence of the lock range of relaxation oscillator with double square-wave injections on the phase difference of the injection signals. The injection ratio is 0.1 .

tone injections is twice that with a single two-tone injection. This observation agrees with the theoretical findings presented earlier.

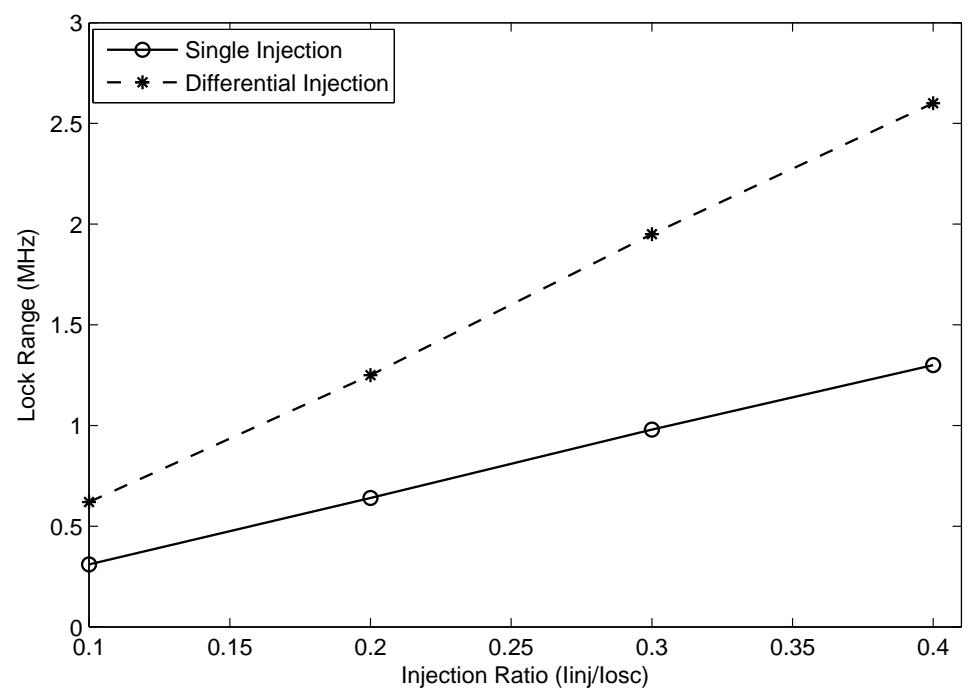

Figure 3.16: Simulated dependence of the lock range of relaxation oscillator with a two-tone sinusoidal injection and double two-tone injections on injection strength. 
Fig. 3.17 compares the lock range of the relaxation oscillator with double single-tone injections, double two-tone injections, and double square-wave injections, all with a 180 degree phase difference. It is seen that the lock range of the oscillator with double squarewave injections is the largest while that with double single-tone injections is the lowest. These observations are in a good agreement with the theoretical results derived earlier.

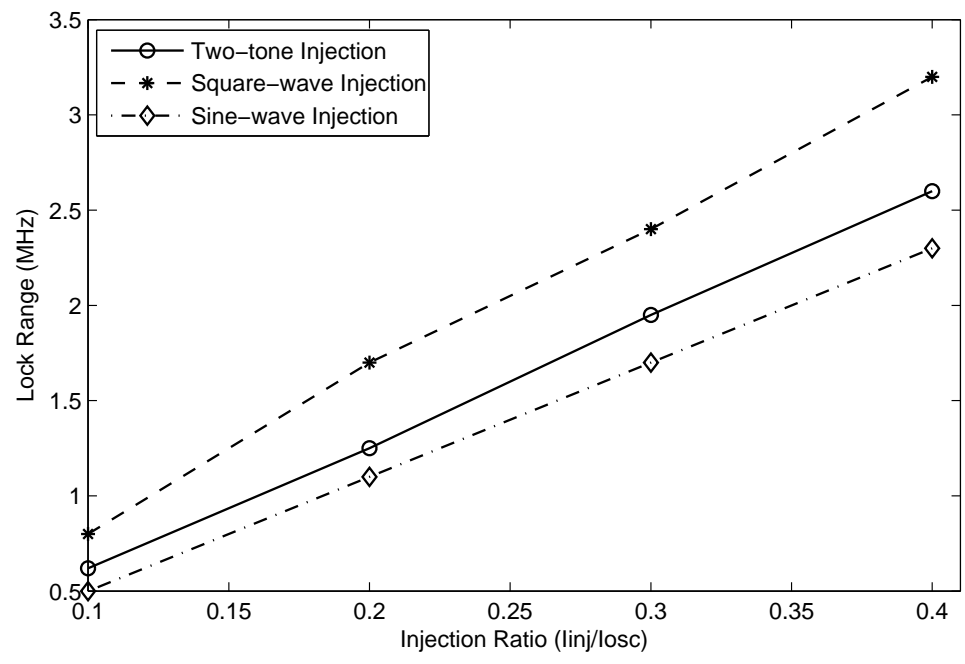

Figure 3.17: Simulated dependence of the lock range of relaxation oscillator with double single-tone injections, double two-tone injections, and double square-wave injections on injection strength.

\subsection{Summary}

By representing non-harmonic oscillators with a set of harmonic oscillators, and using similar approach presented in Chapter 2, the intrinsic relation between the lock range of harmonic oscillators and that of non-harmonic oscillators is obtained. Also, the resemblance of Volterra series analysis for nonlinear circuits is applied to show non-harmonic oscillators with a multi-tone injection exhibit a larger lock range as compared with that with a single-tone injection. Further demonstration shows that non-harmonic oscillators with multiple singletone injections exhibit a larger lock range as compared with that with a single single-tone 
injection. The condition upon which non-harmonic oscillators with multiple single-tone injections exhibits a larger lock range as compared with those with a single-tone injection was obtained. The condition upon which non-harmonic oscillators with multiple multi-tone injections exhibits a larger lock range as compared with those with a single multi-tone injection has also been derived. The above study is critical for some applications. In particular, when the non-harmonic oscillators are employed in passive wireless communications and wireless sensor transceivers, where the power supply is not stabilized, the calibration for local oscillators requires the lock range which has to be wide enough to overcome the frequency drifting. As the characteristics of the oscillating signals are exhibiting more harmonic components, the multiple multi-tones injections are essentially useful to enlarge the lock range. 


\section{Chapter 4}

\section{A Quadrature Charge-Domain Sampling Mixer with Embedded FIR, IIR, and N-Path Filters}

In zero-IF or low-IF RF receivers, a quadrature mixer is commonly employed. The SAW band-pass filter with fixed centre frequency or with a narrow tuning range has to be eliminated for SDR applications. A switched-capacitor based network can be utilized to substitute the SAW filter while proving tunable centre frequency by sampling frequency. A RF receiver employed the windowed integration sampling provides improved attenuation for aliasing and stop-band frequency. In this chapter, a programmable quadrature chargedomain sampling mixer with embedded FIR, IIR, and 4-path band-pass filters is designed. The remainder of the chapter is organized as the followings: Section 4.1 investigates the principles of periodic impulse sampling, periodic windowed sampling, and periodic N-path windowed sampling, and their characteristics are compared. Section 4.2 provides a detailed mathematical treatment of charge-domain windowed samplers. In Section 4.3, a quadrature charge-domain sampler with embedded FIR, IIR, and 4-path band-pass filters is proposed and its performance is investigated in detail. Section 4.4 presents the measurement results. A summary is provided in Section 4.5. 


\subsection{N-path Filters}

\subsubsection{Periodic Impulse Sampling}

Consider the time-varying network shown in Fig. 4.1 where the output of the linear time-invariant (LTI) network with impulse response $h_{B B}(t)$ and bandwidth $\Delta \omega$ is sampled by periodic impulse function $\delta_{T}(t)=\sum_{n=-\infty}^{\infty} \delta\left(t-n T_{s}\right)$ where $\delta(t)=1$ if $t=0$ and 0 otherwise, and $T_{s}$ is the period. Because $\mathcal{F}\left[\delta_{T}(t)\right]=\frac{2 \pi}{T_{s}} \sum_{n=-\infty}^{\infty} \delta\left(\omega-n \omega_{s}\right)$ where $\mathcal{F}[]$ is Fourier transform operator and $\omega_{s}=2 \pi / T_{s}$.

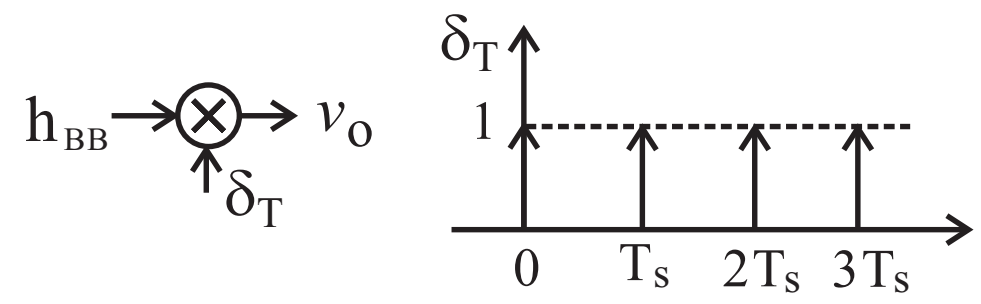

Figure 4.1: Periodic impulse sampling of band-limited signals.

The output is given by $V_{o}(\omega)=H_{B B}(\omega) \otimes \mathcal{F}\left[\delta_{T}(t)\right]$ where $\otimes$ is convolution operator and $H_{B B}(\omega)$ is the Fourier transform of $h_{B B}(t)$. It follows that

$$
V_{o}(\omega)=\frac{2 \pi}{T_{s}} \sum_{n=-\infty}^{\infty} H_{B B}\left(\omega-n \omega_{s}\right)
$$

If $\omega_{s} \geq 2 \Delta \omega$, the frequency characteristic of the LTI network will repeat every $\omega_{s}$ with no spectrum overlap, as shown in Fig. 4.2(a). Periodic impulse sampling converts a low-pass filer to band-pass filters. The spectral profile of the band-pass filters (bandwidth, stop-band attenuation,...) is identical to that of the low-pass while the center frequency can be tuned by varying $\omega_{s}$. The band-pass filter with its center frequency at $\omega_{s}$ will have quality factor $Q=\omega_{s} /(2 \Delta \omega)$. For example, if $\Delta f=1 \mathrm{MHz}$ and $f_{s}=1 \mathrm{GHz}$, we have $Q=500$. 


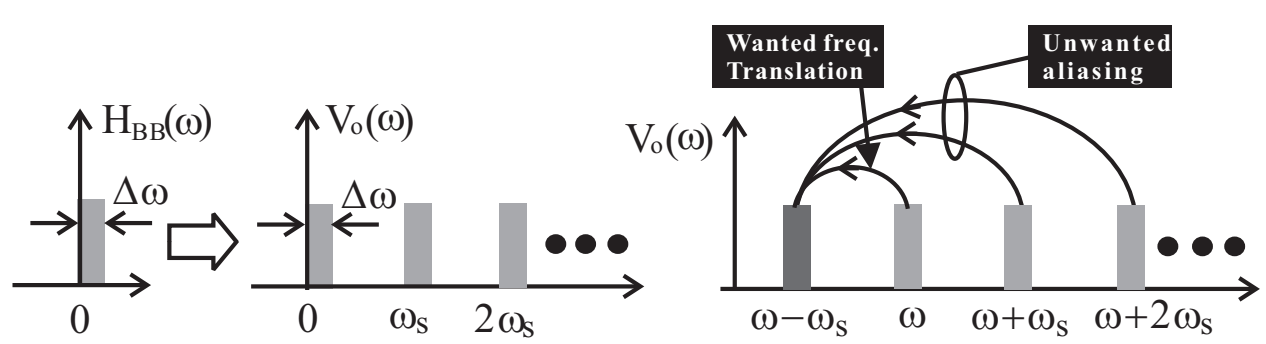

(a)

(b)

Figure 4.2: (a) Periodic impulse sampling of band-limited signals. Baseband signal at $\omega$ is replicated to sidebands at $\omega+n \omega_{s}$. (b) Periodic impulse sampling of broadband signals. The sideband components of input at $\omega+n \omega_{s}$ are aliased back to the baseband at $\omega$.

Consider that the input at $\omega$ and with bandwidth $\Delta \omega \leq \omega_{s} / 2$ and at the same time other unwanted tones exist at $\omega+n \omega_{s}$. Assume the desired output is at $\omega-\omega_{s}$ obtained via mixing. Tones at $\omega+n \omega_{s}$ will be aliased back to $\omega-\omega_{s}$, as shown in Fig. 4.2(b). Mechanisms that suppress the effect of the aliasing tones are needed.

\subsubsection{Periodic Windowed Sampling}

A time-varying network is shown in Fig. 4.3 where the output of the LTI network having a low-pass characteristic with bandwidth $\omega_{B}$ is sampled by periodic windowed function $s_{T}(t)=1$ if $n T_{s}+\sigma_{k-1} \leq t<n T_{s}+\sigma_{k}$ and 0 otherwise. $\sigma_{k}$ and $\sigma_{k-1}$ are explicitly defined in Fig. 4.3. Since $s_{T}(t)$ is periodic with period $T_{s}$, it can be represented by Fourier series

$$
s_{T}(t)=\sum_{n=-\infty}^{\infty} S_{n} e^{j n \omega_{s} t}
$$

where

$$
\begin{aligned}
S_{n} & =\frac{1}{T_{s}} \int_{0}^{T_{s}} s_{T}(t) e^{-j n \omega_{s} t} d t \\
& =\frac{\tau}{T_{s}} e^{-j n \omega_{s} \sigma_{k-1}} e^{-j n \omega_{s} \tau / T_{s}} \operatorname{sinc}\left(\frac{n \pi \tau}{T_{s}}\right) .
\end{aligned}
$$




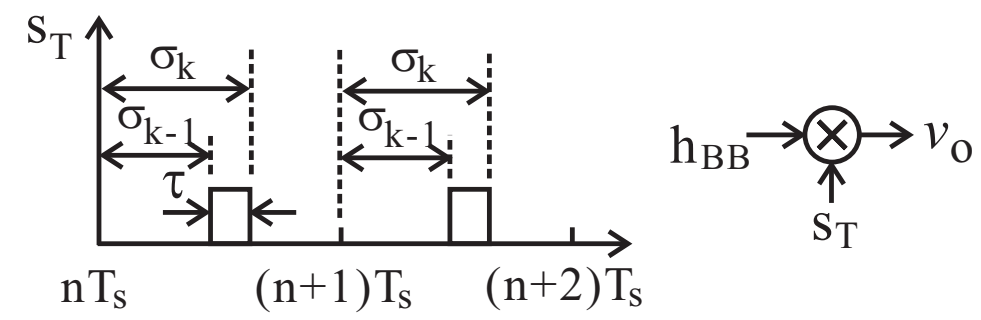

Figure 4.3: Periodic windowed sampling of band-limited signals.

Fourier transform of $s_{T}(t)$ can be obtained directly through (4.2) and (4.3)

$$
\begin{aligned}
S_{T}(\omega)= & \frac{2 \pi \tau}{T_{s}} \sum_{n=-\infty}^{\infty} e^{-j n \omega_{s} \sigma_{k-1}} e^{-j n \omega_{s} \tau / T_{s}} \operatorname{sinc}\left(\frac{n \pi \tau}{T_{s}}\right) \\
& \delta\left(\omega-n \omega_{s}\right) .
\end{aligned}
$$

Fourier transform of $v_{o}(t)=h_{B B}(t) s_{T}(t)$ is obtained from

$$
\begin{aligned}
V_{o}(\omega)= & H_{B B}(\omega) \otimes S_{T}(\omega) \\
= & \frac{\tau}{T_{s}} \sum_{n=-\infty}^{\infty} e^{-j n \omega_{s} \sigma_{k-1}} e^{-j n \omega_{s} \tau / 2} \operatorname{sinc}\left(\frac{n \pi \tau}{T_{s}}\right) \\
& H_{B B}\left(\omega-n \omega_{s}\right) .
\end{aligned}
$$

Consider $\omega_{s} \geq 2 \Delta \omega$ without interferences. Periodic windowed sampling converts it to a set of band-pass filters. Unlike periodic impulse sampling that has a uniform scaling factor $2 \pi / T_{s}$, periodic windowed sampling has a low-pass scaling factor sinc $\left(n \pi \tau / T_{s}\right)$, as shown in Fig. $4.4(\mathrm{a})$.

For the case of $\omega_{s} \geq 2 \Delta \omega$ with interferences at $\omega+n \omega_{s}(n \neq 1)$, if the desired output is at $\omega+\omega_{s}$, the baseband tone at $\omega$ and the interferences at $\omega+n \omega_{s}$ will be converted to the desired output at $\omega+\omega_{s}$. Since $\left|e^{-j n \omega_{s} \sigma_{k-1}}\right|=\left|e^{-j n \omega_{s} \tau / 2}\right|=1$, the aliasing effect at $\omega+n \omega_{s}$ will be magnitude-shaped by the scaling factor $\operatorname{sinc}\left(n \pi \tau / T_{s}\right)$.

Unlike periodic impulse sampling, periodic windowed sampling introduces nulls. For the interferences reside at the nulls, they will be completely suppressed (ideally). As usual, the desired channel occupies a frequency band $\Delta \omega$ rather than a single frequency, although 

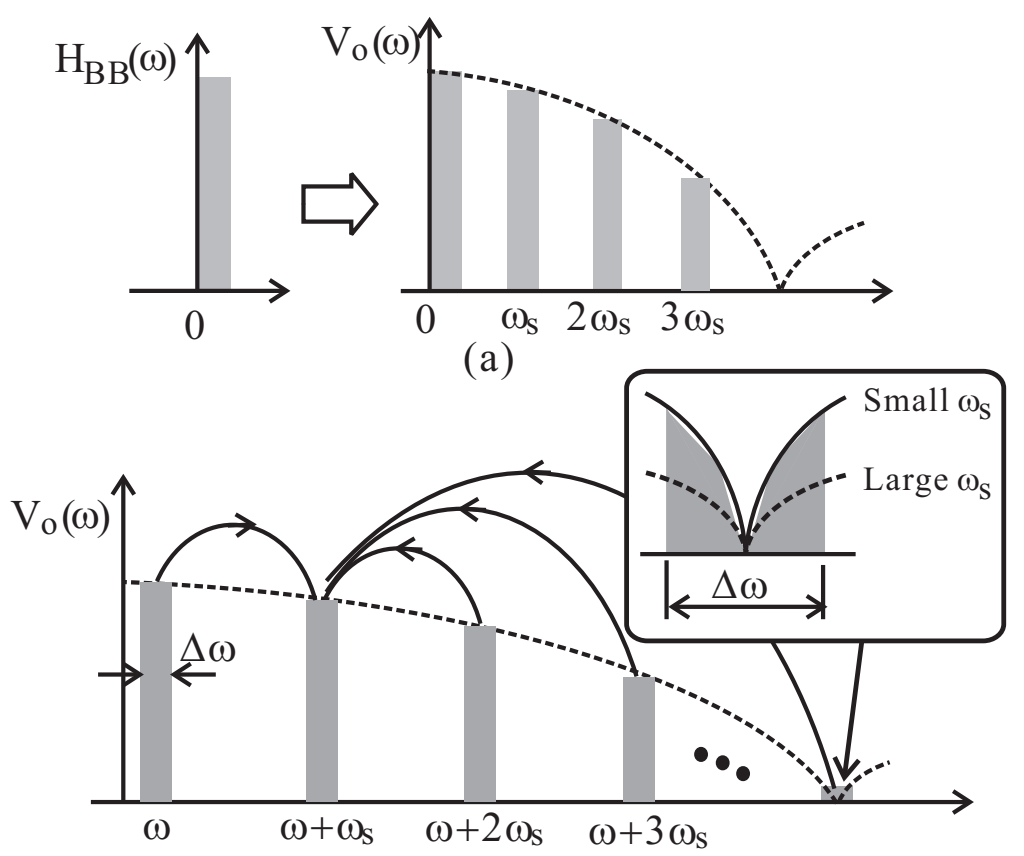

(b)

Figure 4.4: (a) Periodic windowed sampling of narrow-band signals without interferences. (b) Periodic windowed sampling of band-limited signals with interferences. Interferences at $\omega+n \omega_{s}$ are aliased back to the desired baseband.

nulls will be most effective in suppressing aliasing tones at $\omega+n \omega_{s}$, those in the vicinity of $\omega+n \omega_{s}$ will be suppressed but with less attenuation, as illustrated graphically in Fig. 4.4(b). Also, increasing $\omega_{s}$ will improve the attenuation of tones in the vicinity of the nulls. This, however, is at the cost of increased power consumption.

\subsubsection{Periodic N-Path Windowed Sampling}

N-path filters introduced in 1960s [111] offer the intrinsic advantages of a programmable center frequency, programmable bandwidth, low-cost, and an interleaved operation enabling low-speed circuits to process high-frequency inputs. An interleaved N-path filter is shown in Fig. 4.5 with a total of $N$ identical networks sampled by window function $s_{k}(t)$ defined as $s_{k}(t)=1$ if $n T_{s}+\sigma_{k-1} \leq t<n T_{s}+\sigma_{k}$ and 0 otherwise. Since $s_{k}(t)$ is periodic with period $T_{s}$, we have $s_{k}(t)=\sum_{n=-\infty}^{\infty} S_{k, n} e^{j n \omega_{s} t}$ where $S_{k, n}=\frac{1}{N} \operatorname{sinc}\left(\frac{n \pi}{N}\right) e^{j \frac{n \pi}{N}} e^{-j \frac{2 n k \pi}{N}}$. Note that $\tau=T_{s} / N$ 
was utilized. Fourier transform of $s_{k}(t)$ is given by

$$
S_{k}(\omega)=\frac{2 \pi}{N} \sum_{n=-\infty}^{\infty} \operatorname{sinc}\left(\frac{n \pi}{N}\right) e^{j \frac{n \pi}{N}} e^{-j \frac{2 n k \pi}{N}} \delta\left(\omega-n \omega_{s}\right)
$$
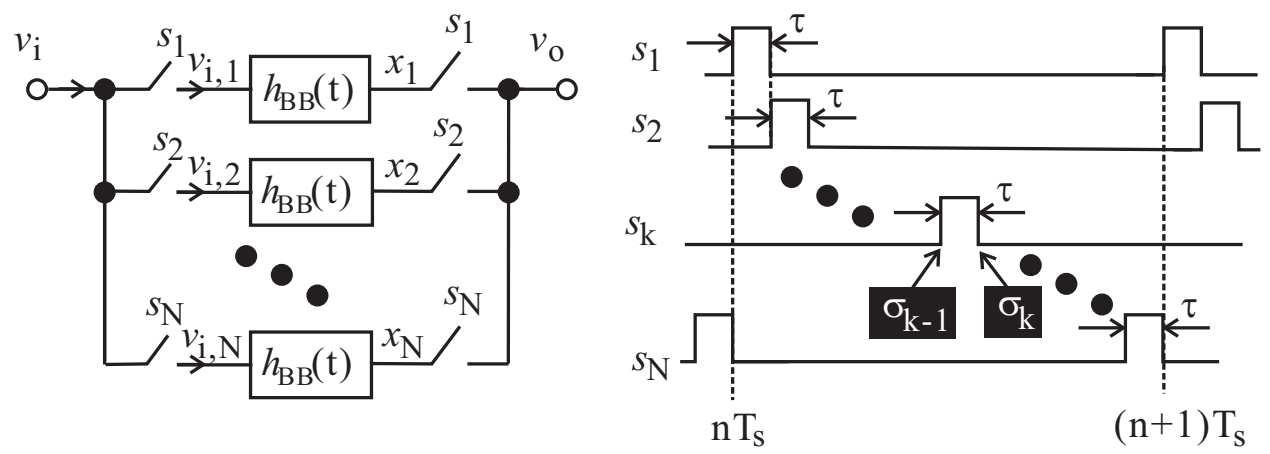

Figure 4.5: N-path filter. Sampling mixers are implemented using switches drived by a sampling clock with $N$ non-overlapping phases. Non-overlapping uniform sampling pulse width: $\tau=T_{s} / N . \sigma_{k}=\sigma_{k-1}+\tau$ and $\sigma_{k-1}=n T_{s}+(k-1) \tau$.

Fourier transform of $v_{i, k}=v_{i}(t) s_{k}(t)$ is obtained from convolution

$$
V_{i, k}(\omega)=\frac{1}{N} \sum_{n=-\infty}^{\infty} \operatorname{sinc}\left(\frac{n \pi}{N}\right) e^{j \frac{n \pi}{N}} e^{-j \frac{2 n k \pi}{N}} V_{i}\left(\omega-n \omega_{s}\right)
$$

where $V_{i}(\omega)$ is the Fourier transform of $v_{i}(t)$. The output of $k$ th LTI block before the second mixer $x_{k}(t)$ is given by $x_{k}(t)=v_{i, k}(t) \otimes h_{B B}(t)$ and its Fourier transform $X_{k}(\omega)$ is given by

$$
\begin{aligned}
X_{k}(\omega)= & \frac{1}{N} \sum_{n=-\infty}^{\infty} \operatorname{sinc}\left(\frac{n \pi}{N}\right) e^{j \frac{n \pi}{N}} e^{-j \frac{2 n k \pi}{N}} \\
& V_{i}\left(\omega-n \omega_{s}\right) H_{B B}(\omega) .
\end{aligned}
$$


The output in $k$ th phase is obtained from

$$
\begin{aligned}
v_{o, k}(t) & =x_{k}(t) s_{k}(t) \\
& =\frac{1}{N} \sum_{m=-\infty}^{\infty} \operatorname{sinc}\left(\frac{m \pi}{N}\right) e^{j \frac{m \pi}{N}} e^{-j \frac{2 m k \pi}{N}} x_{k}(t) e^{j m \omega_{s} t}
\end{aligned}
$$

The index $m$ associated with the second set of switches is used in (4.9). It is straightforward to show that

$$
\begin{aligned}
& V_{o}(\omega)=\sum_{k=1}^{N} \mathcal{F}\left[v_{o, k}(t)\right] \\
& \frac{1}{N} \sum_{k=1}^{N} \sum_{m=-\infty}^{\infty} \operatorname{sinc}\left(\frac{m \pi}{N}\right) e^{j \frac{m \pi}{N}} e^{-j \frac{2 m k \pi}{N}} X_{k}\left(\omega-m \omega_{s}\right) \\
& =\left(\frac{1}{N}\right)^{2} \sum_{k=1}^{N} \sum_{m, n=-\infty}^{\infty} \operatorname{sinc}\left(\frac{m \pi}{N}\right) \operatorname{sinc}\left(\frac{n \pi}{N}\right) e^{j \frac{(m+n) \pi}{N}} \\
& e^{-j \frac{2(m+n) k \pi}{N}} V_{i}\left[\omega-(m+n) \omega_{s}\right] H_{B B}\left(\omega-n \omega_{s}\right),(m+n=K N, K=0,1,2, \ldots) .
\end{aligned}
$$

Since $\left|e^{j(m+n) \pi / N}\right|=1$ and $\left|e^{-j 2(m+n) k \pi / N}\right|=1$, we obtain the magnitude of $V_{o}(\omega)$ from $(4.10)$

$$
\begin{aligned}
V_{o, m}(\omega)= & \frac{1}{N^{2}} \sum_{m, n=-\infty}^{\infty} \operatorname{sinc}\left(\frac{m \pi}{N}\right) \operatorname{sinc}\left(\frac{n \pi}{N}\right) \\
& V_{i}\left[\omega-(m+n) \omega_{s}\right] H_{B B}\left(\omega-n \omega_{s}\right),(m+n=K N, K=0,1,2, \ldots) .
\end{aligned}
$$

The transfer function of the N-path filter at $\omega$ is obtained by letting $m+n=0$

$$
H_{B P}(\omega)=\frac{V_{o}(\omega)}{V_{i}(\omega)}=\frac{1}{N^{2}} \sum_{n=-\infty}^{\infty} \operatorname{sinc}^{2}\left(\frac{n \pi}{N}\right) H_{B B}\left(\omega-n \omega_{s}\right)
$$

As compared with periodic windowed sampling,

1. Periodic N-path windowed sampling provides a sinc ${ }^{2}$ low-pass characteristic with -40 $\mathrm{dB} /$ dec attenuation to suppress unwanted tones while periodically windowed sampling provides a sinc low-pass characteristic with $-20 \mathrm{~dB} / \mathrm{dec}$ attenuation. 
2. Both periodically windowed sampling and periodic N-path windowed sampling provide nulls to suppress aliasing interferences at and in the vicinity of nulls.

3. For periodic N-path windowed sampling from (4.12), the N-path sampling can be applied to select frequency components near by $\omega_{s}$ in a manner analogous to employing a band-pass filter at the high frequency. Meanwhile the quality factor of the band-pass filter ideally is only determined by the base-band spectral profile, e.g. $H_{B B}$ is a low-pass filter. The center of the band-pass filter is precisely controlled by the $\omega_{s}$.

4. Apart from the band-pass filtering at the desired frequency, $\omega_{s}$, there are also response peaks around all the other harmonics of the switching frequency. In other words, the unwanted signals will be accompanied with the wanted tones through the switching systems. The severe drawback, which can be observed from (4.11), is the folding of interferences at some harmonics of $\omega_{s}$ on the top of the desired signal at $\omega_{s}$. For example, if $\mathrm{N}$ is 4 and the desired signal is located at $\omega_{s}+\omega$, the closest harmonic, which will introduce aliasing signals, is located at $3 \omega_{s}$. And the next will be $5 \omega_{s}$.

5. The location of the nulls in periodic N-path windowed sampling is heavily depending on the number of the paths. In other words, increasing the number of paths moves nulls to high frequencies, lowering the overall fold-over effect of aliasing interferences at and near the nulls due to the increasing attenuation of these unwanted tones at high frequencies. It is of interest to notice that the harmonic rejection at all the other harmonics is also decreased by increasing the number of paths. Take a 4-path and 8-path filter for example, the $3^{r d}$-harmonic of the 4 -path filter is located at $0.75 * 4 \omega_{s}$ with $20 \mathrm{~dB}$ insertion loss. The $3^{r d}$-harmonic of the 8-path filter, however, is located at $0.375 * 8 \omega_{s}$ with only $4 \mathrm{~dB}$ attenuation.

The following example shown in Fig. 4.6(a) [90] illustrates a simplified single-ended $\mathrm{N}$-path filter, where $\mathrm{N}$ is 4 and $\tau$ is $T_{s} / 4$. The targeting signal is at in the vicinity of $\omega_{s}$. The input voltage $v_{i}$ is converted to current $i_{i}$ first. $i_{i}$ is then switched by $s_{k}(t)$ and integrated onto $C_{B}$. Fig. 4.6(b) shows the non-overlapping multi-phase clocking scheme for the switching system. In other words, for every periodic switching, only one capacitor is being charged or 
discharged. The the output impedance of the transconductor and single-ended capacitors form a low-pass filter with time constant $\tau_{B}=R_{\text {out }} C_{B}$ where $R_{\text {out }}$ is the output impedance of the transconductor, if the ON-resistance of the switches is sufficiently small. From 4.12, $H_{B B}$ is denoted by a first-order integrator, $H_{B B}=\frac{R_{\text {out }}}{1+j \omega R_{\text {out }} C_{B}}$.

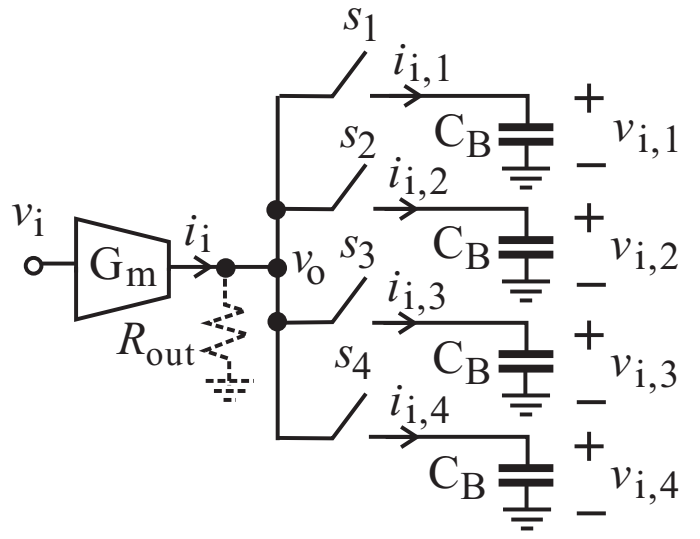

(a)

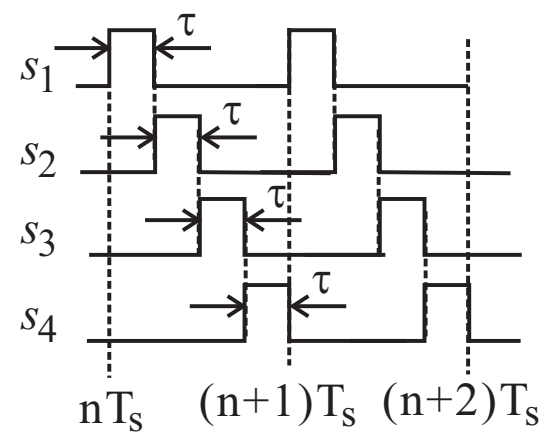

(b)

Figure 4.6: Single-ended 4-path filter.

A careful inspection of Fig. 4.6 reveals that it realizes the same function as Fig. 4.5 does. The sampled current $i_{i, k}(t)=i_{i}(t) s_{k}(t)$ produces voltage $v_{i, k}(t)=i_{i, k}(t) \otimes z_{B}(t)$ across the capacitor where $z_{B}(t)$ is the impulse response of the load. As the charge on each capacitor has nowhere to flow except for flowing back to the input port of the switch, which is same as $x_{k}$ in Fig. 4.5 passing through second mixer. $v_{i, k}(t)$ is then sampled by the switches to produce $v_{o}(t): v_{o}(t)=\sum_{k=1}^{4} v_{i, k}(t) s_{k}(t) . v_{o}(t)$ can be obtained by the network shown in Fig. 4.7.

The whole concept of substituting the conventional band-pass filter can be understood in the form of impedance looking into the input the switching system. If the baseband impedance around DC presents a very large value, the impedance at $\omega_{s}$ will be very large due to the switching system. From Fig. 4.6, the baseband low-pass filter is then converted to a highfrequency band-pass filter. The out of band of the band-pass filter exhibits a low impedance path to those interferences residing at the frequency offsets from $\omega_{s}$ while the desired signal 

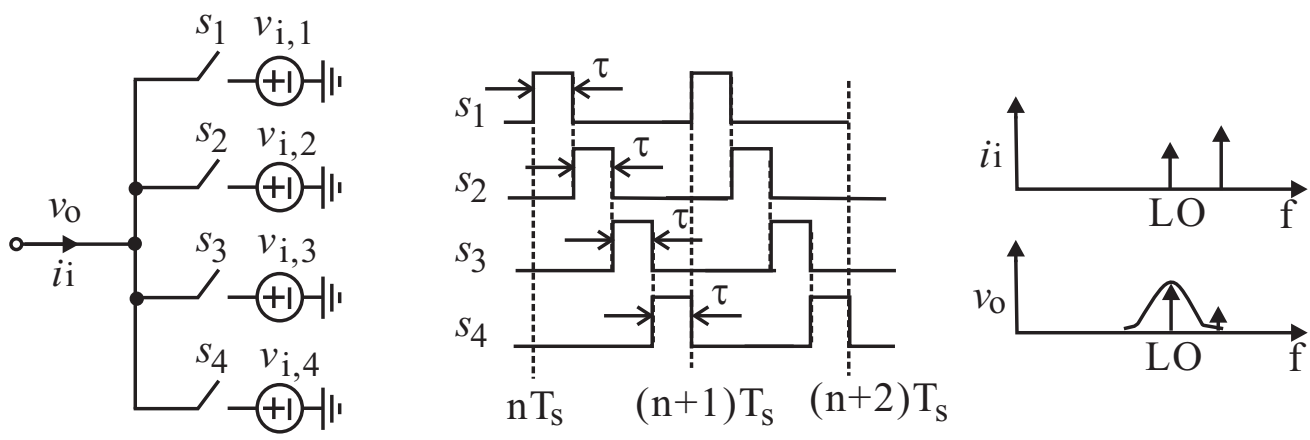

Figure 4.7: Single-ended 4-path filter.

will be picked up. The quality factor of this type filter is feasible to be designed over 1000 depending on the bandwidth and the center frequency. Fig. 4.8 plots the simulated PSS-PAC frequency response of the 4-path band-pass filter shown in Fig. 4.7 with $G_{m}=1$ $\mathrm{mS}, f_{s}=100 \mathrm{MHz}$ with $25 \%$ duty cycle, and $C_{B}=40 \mathrm{pF}$. It is seen that the $4^{\text {th }}$ and $8^{\text {th }}$ harmonics vanish due to the frequency-shaping in (4.12). Also observed is the sinc envelope of the frequency response with notches at $4 f_{s}, 8 f_{s}, \ldots$ The 4-path band-pass filter can also be implemented differentially. In this case, the differential form removes all even number harmonics components and DC.

\subsection{Charge-Domain Windowed Sampling}

\subsubsection{Sinc Low-Pass Filter}

This section examines the operation of the charge-domain sampler shown in Fig. 4.9. The input voltage signal is converted to a current through a $G_{m}$ cell with the transconductance gain $G_{m}$, followed by a switched-capacitor(SC) circuit. It is worth to mention that the realization of integrator is possible to use OPAMP-based integrator. Each period of the sampling clock consists of three phases : the reset phase $\left(s_{r}=1\right)$ where sampling capacitor $C_{s}$ is reset, the integration phase $\left(s_{i}=1\right)$ where the output current of the transconductor is integrated onto the sampling capacitor, $C_{s}$, over $T_{i}$ period, and the readout phase $\left(s_{o}=1\right)$ at a rate $f_{s}=1 / T_{s}$, as shown in Fig. 4.9. The reset operation ensures that there is no relation between the output of the sampler at the end of adjacent sampling periods. Let 


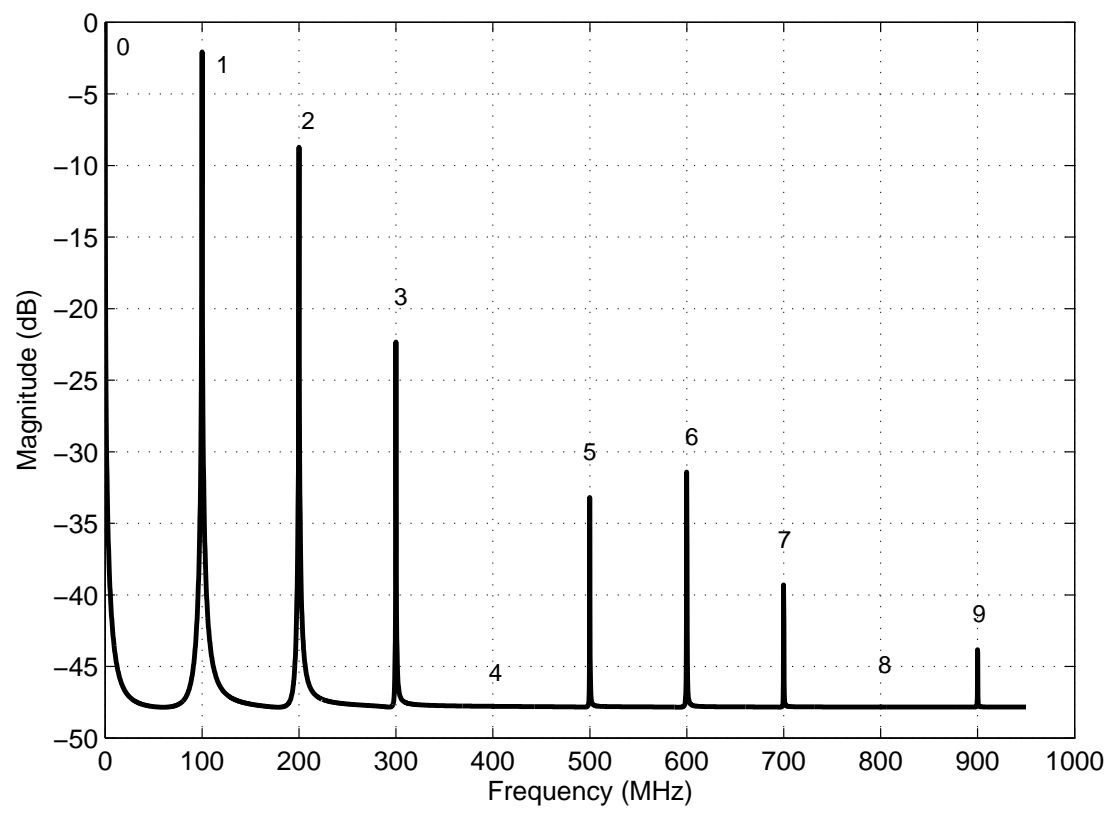

Figure 4.8: Frequency response of single-ended 4-path filter in Fig. 4.6.

$v_{i}(t)=e^{j \omega t}$. Because $v_{i}(t)$ is a single tone of unit amplitude, the corresponding output is the transfer function of the system. The output voltage at the end of the integration phase $T_{i}$ is given by

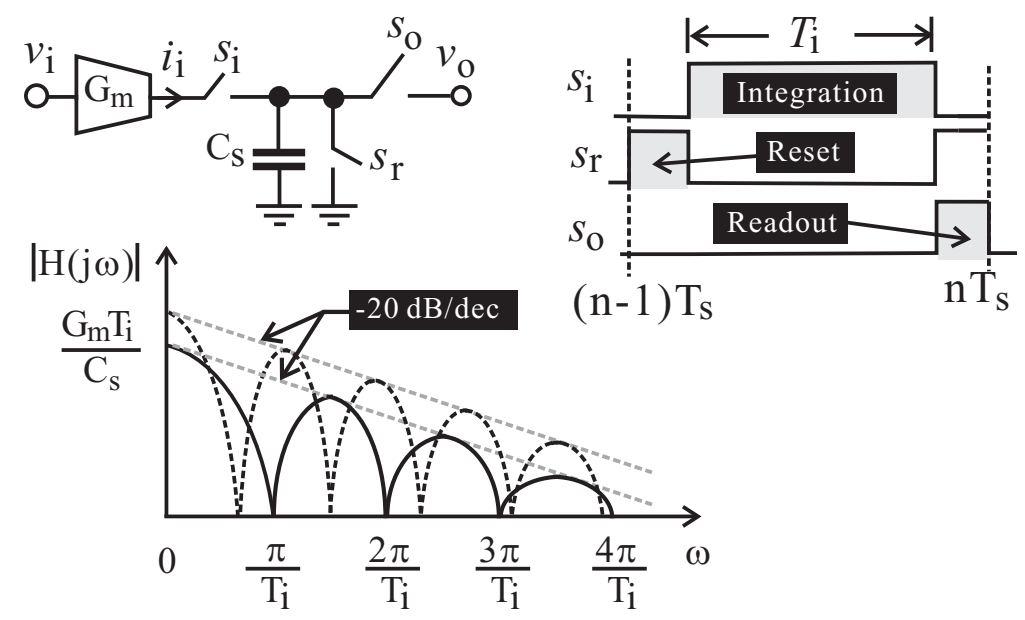

Figure 4.9: Charge-domain sampling. Solid line : Small $T_{i}$. Dashed line : Large $T_{i}$. 


$$
\begin{aligned}
v_{o}\left(n T_{s}\right) & =\frac{G_{m}}{C_{s}} \int_{n T_{s}-T_{i}}^{n T_{s}} e^{j \omega t} d t \\
& =\frac{G_{m} T_{i}}{C_{s}} e^{j n \omega T_{s}} e^{-j \omega T_{i} / 2} \operatorname{sinc}\left(\frac{\omega T_{i}}{2}\right)
\end{aligned}
$$

The ideal frequency response of the output signal as a function of input signal from (4.13) can be expressed as

$$
H_{\text {sinc }}(j \omega)=\frac{G_{m} T_{i}}{C_{s}}\left|\operatorname{sinc}\left(\frac{\omega T_{i}}{2}\right)\right| .
$$

$\left|H_{\operatorname{sinc}}(j \omega)\right|$ is sketched in Fig. 4.9. It is of interest to notice that the charge-domain windowed sampling process can be represented by two independent signal processing operations, a continuous-time (CT) sinc filtering due to integration of the signal current within a widowed period and a sampling process at the rate, $f_{s}=1 / T_{s}$. The preceding results are commented as:

1. Charge-domain windowed sampling provides a sinc low-pass characteristic with nulls at $\omega=n \pi / T_{i}$ and a $-20 \mathrm{~dB} / \mathrm{dec}$ attenuation. The signal bandwidth is determined by $T_{i}$, and is approximately $0.44 / T_{i}$, shown in Fig. 4.10 .

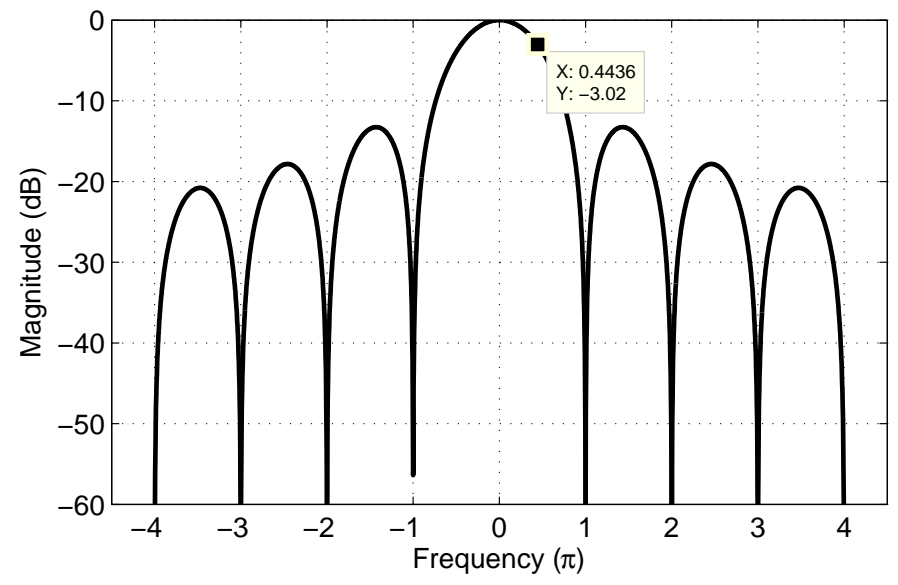

Figure 4.10: Gain-normalized transfer function of sinc. 
2. The gain of the filter in the passband $0 \leq f \leq 0.44 / T_{i}$ is not constant but rather drops with frequency in a sinc fashion. The low-pass obtained from charge-domain windowed sampling therefore does not have a flat passband.

3. The largest gain occurs at $\mathrm{DC}$ and is given by $G_{m} T_{i} / C_{s}$. Increasing $T_{i}$ not only reduces the bandwidth of the charge-domain sampler, it also boosts the DC gain. Also the larger the sampling capacitor, the lower the gain. On the other hand, the larger the transconductance, the larger the gain. The latter, however, is accompanied with more power consumption.

4. Since nature of sinc function is low-pass and the attenuation in the main lobe is small, the desired output should be placed inside the main lobe, preferably close to DC. This requires that $\omega \ll \omega_{i}$ or equivalently $T_{i} \ll T$ where $\omega_{i}=\pi / T_{i}$ is the frequency of the first null.

5. For a given input at frequency $\omega$, if the desired output is at DC, the sampling frequency $\omega_{s}=2 \pi / T_{s}$ is chosen such that $\omega-\omega_{s}=0$. Since $T_{s}=T_{r}+T_{i}+T_{o}$ where $T_{r}$ and $T_{o}$ are the reset time and readout time, respectively, $T_{i}$ will therefore vary with $T_{s}$. This will in turn affect the gain of the sampler given by $H_{\text {sinc }}(0)=G_{m} T_{i} / C_{s}$ unless $G_{m}$ is also varied accordingly such that $G_{m} T_{i}$ remains unchanged. Since most receivers require an Automatic Gain Control (AGC), this gain variation can be tuned out as part of the AGC function. As a result, no additional hardware is needed. Alternatively, the gain of the transconductor can also be varied..

6. If the desired output is at DC, assume $T_{i}=T_{s}$, aliasing interferences at $n \omega_{i}$ will be fully attenuated. If the desired signal is close to DC, aliasing interferences are located near the nulls and will also be attenuated by the nulls, however, by a less amount.

7. As demonstrated in $[86,112]$, the depth of the nulls is greatly affected by the imperfection of the sampler, specifically, the finite output impedance of the transconductor as the finite output impedance of the transconductor reduces the effective current integrated onto the sampling capacitor. As a result, the effectiveness of the nulls in suppressing aliasing interferers is reduced. 
The importance of the concept of the charge-domain sampler in Fig. 4.9 is that the current integration provides an elementary low-pass sinc response and the input signal bandwidth is primarily set by integrating time, $T_{i}$. On the contrary, in voltage sampler, the input signal bandwidth is limited by the time constant, $\tau=R C$, where $\mathrm{R}$ is the ONresistance of the switches and $\mathrm{C}$ is the sampling capacitance. Given the same bandwidth for both two samplers, the performance of low-pass sinc filter ideally outperforms the simple low-pass RC filter as the unwanted signal at the notches can be suppressed significantly. The simple structure of charge-domain sampler in Fig. 4.9, however, does not take full advantage of anti-aliasing sinc filter since the $T_{i}$ is directly related to $T_{s}$, i.e. $T_{s}$ is not equal to $T_{i}$. In theory, $T_{i}$ and $T_{s}$ are two distinct parameters. $T_{s}$ determines the spectral location and Nyquist bandwidth of the sampled signal while $T_{i}$ sets the nulls. To minimize the fold-over signal on top of the desired signal, it is preferable to set $T_{i}=T_{s}$ when the sampler is utilized in the baseband sampling. The need of reset and read out period in sampling operations, however, cannot be avoided. This issue is resolved by using time interleaved charge-domain sampling circuits, illustrated in Fig. 4.11.
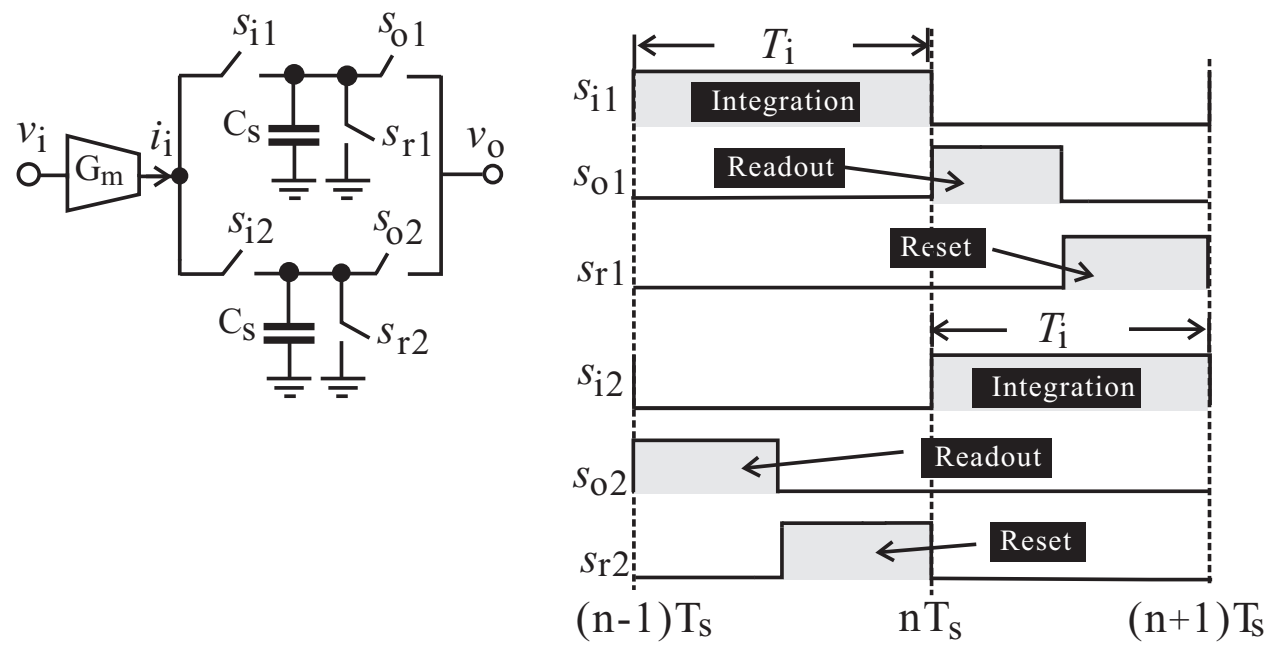

Figure 4.11: Time interleaved charge-domain sampling.

In this case, one integrator is involved in integrating the input current while the other one is in the phase of read out and reset and vice versa. Therefore, the charges in every integration 
period is transferred to the subsequent stage. As a result, the sampling period of the sampler is $T_{s}=T_{i}$, leading to place the notches of the sinc response on top of the multiples of $1 / T_{s}$, which ideally remove the aliasing effect near by DC. If longer time slots for read out and reset are required, more than two paths can be used to further relax the timing requirement.

\subsubsection{FIR Filter}

$-20 \mathrm{~dB} /$ dec attenuation provided by elementary charge-domain windowed sampling is inadequate in suppressing unwanted channels. It was shown in [112] that sinc low-pass provided by the charge-domain windowed sampling can be greatly enhanced by partitioning the integrating phase into multiple sub-integration phases, as shown in Fig. 4.12. Further, for $k$ th integration phase, a weighting factor $h_{k}$ is assigned. The current to be integrated on the sampling capacitor $C_{s}$ during $k$ th sub-phase is given by $i_{i, k}(t)=G_{m} v_{i} h_{k}$. Let $v_{i}(t)=e^{j \omega t}$. The output voltage of the sampling capacitor at the end of $n$th sampling period is given by
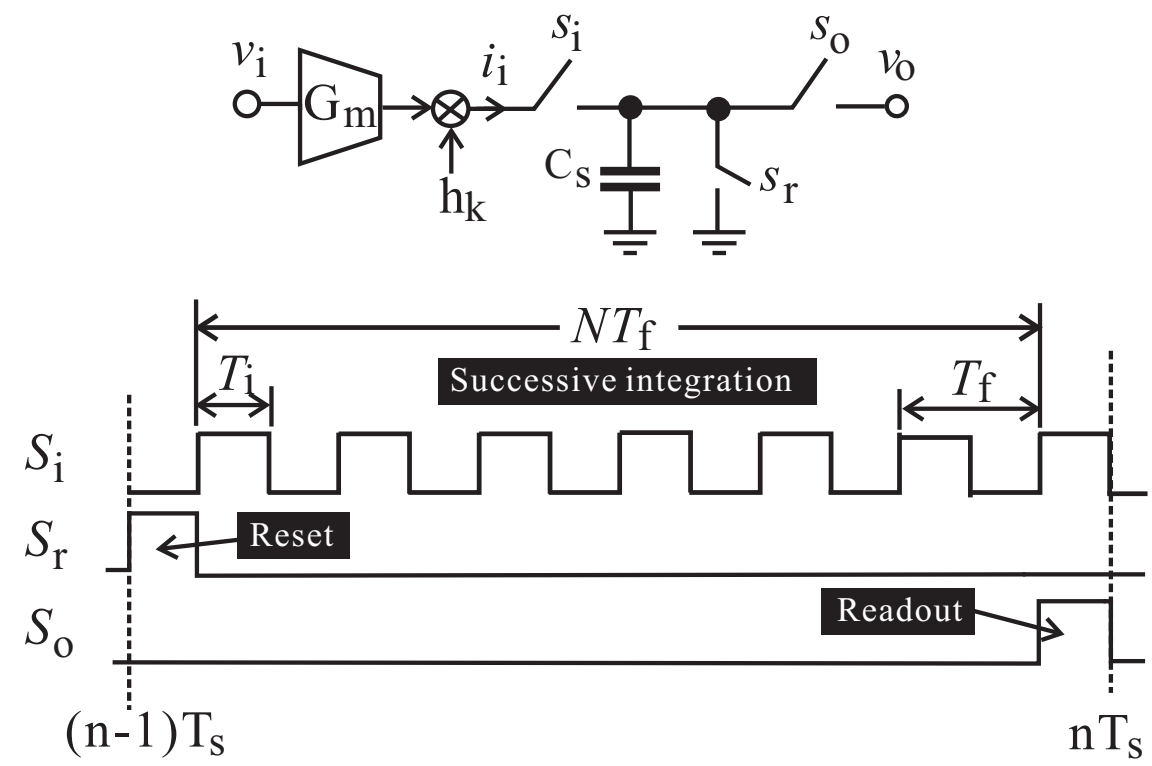

Figure 4.12: Charge-domain sampler with built-in FIR filter. 


$$
\begin{aligned}
v_{o}\left(n T_{s}\right) & =\frac{G_{m}}{C_{s}} \sum_{k=0}^{N-1}\left(h_{k} \int_{n T_{s}-k T_{f}-T_{i}}^{n T_{s}-k T_{f}} e^{j \omega t} d t\right) \\
& =\frac{G_{m}\left(1-e^{-j \omega T_{i}}\right)}{j \omega C_{s}} \sum_{k=0}^{N-1} h_{k} e^{j n \omega T_{s}} e^{-j \omega k T_{f}} .
\end{aligned}
$$

Defining $z=e^{j \omega T_{f}}$ and noting $\left|e^{j n \omega T_{s}}\right|=1$, we obtain magnitude transfer function

$$
|H(j \omega)|=\frac{G_{m} T_{i}}{C_{s}}\left|\operatorname{sinc}\left(\frac{\omega T_{i}}{2}\right) \sum_{k=0}^{N-1} h_{k} z^{-k}\right|=H_{\text {sinc }} H_{F I R}
$$

where $H_{\text {sinc }}$ was defined in (4.14) and $H_{F I R}=\sum_{k=0}^{N-1} h_{k} z^{-k}$. Eq.(4.16) shows that two distinct filtering functions, namely, a CT sinc low-pass and a DT FIR filter of order $N$, are realized simultaneously. The continuous-time sinc low-pass is due to the continuous integration of the input current over $\left[0, T_{i}\right]$ while the discrete time FIR is due to multiple integrations in one $T_{s}$. A special case is when $h_{k}=1, k=0,1,2, \ldots$, the unity tap weight of the FIR filter gives rise to a temporal moving average characteristic. Assume $T_{f} / T \ll 1$. This is often true since $T_{s}$ is chosen close to $T$ in order to generate a near DC output while $T_{s}=N T_{f}$ with $N$ typically large in order to increase the order of the FIR filter. It follows that $z^{-1}=e^{-j 2 \pi T_{f} / T} \approx 1-j 2 \pi \frac{T_{f}}{T}$. As a result, Eq.(4.16) becomes

$$
H_{F I R}=\frac{1-z^{-N}}{1-z^{-1}}=N e^{-j N \pi T_{f} / T} \operatorname{sinc}\left(N \pi \frac{T_{f}}{T}\right)
$$

Eq.(4.17) shows that the temporal moving average filter implements a sinc low-pass with nulls at $\omega=(n / N) \omega_{f}$ where $\omega_{f}=2 \pi / T_{f}$ and $n=1,2,3, \ldots$, which is often used in the decimation as anti-aliasing filter. There are total N-1 zeros evenly distributed in the frequency range from DC to $1 / T_{f}$, resulting in multiple notches. The optimal output sampling rate is located at the multiple zeros. Therefore, the number of $\mathrm{N}$ limits the decimation ratio. Also, it is evident that the larger the value of $N$, the narrower the nulls and the less the suppression of unwanted channels in the vicinity of the nulls. Reducing $N$, though improving attenuation, 
increases the readout rate. Also observed from (4.17) is that the larger the value of $N$, the larger the voltage gain provided by the temporal moving average filter. Fig. 4.13 and Fig. 4.14 show the comparison of $\mathrm{N}=10$ and $\mathrm{N}=5$. The overall magnitude transfer function of Fig. 4.12 is given by

$$
|H(j \omega)|=\frac{N G_{m} T_{i}}{C_{s}}\left|\operatorname{sinc}\left(\frac{\omega T_{i}}{2}\right) \operatorname{sinc}\left(\frac{N \pi T_{f}}{T}\right)\right| .
$$

and is plotted in Fig. 4.15. It can be expected that the joint effect of $\operatorname{sinc}\left(\frac{\omega T_{i}}{2}\right)$ and sinc $\left(\frac{N \pi T_{f}}{T}\right)$ will not only increase the attenuation in the stop-band, thereby improving the suppression of unwanted channels, it will also increase the depth of the nulls, improving the suppression of aliasing interferers at and in the vicinity of the nulls.

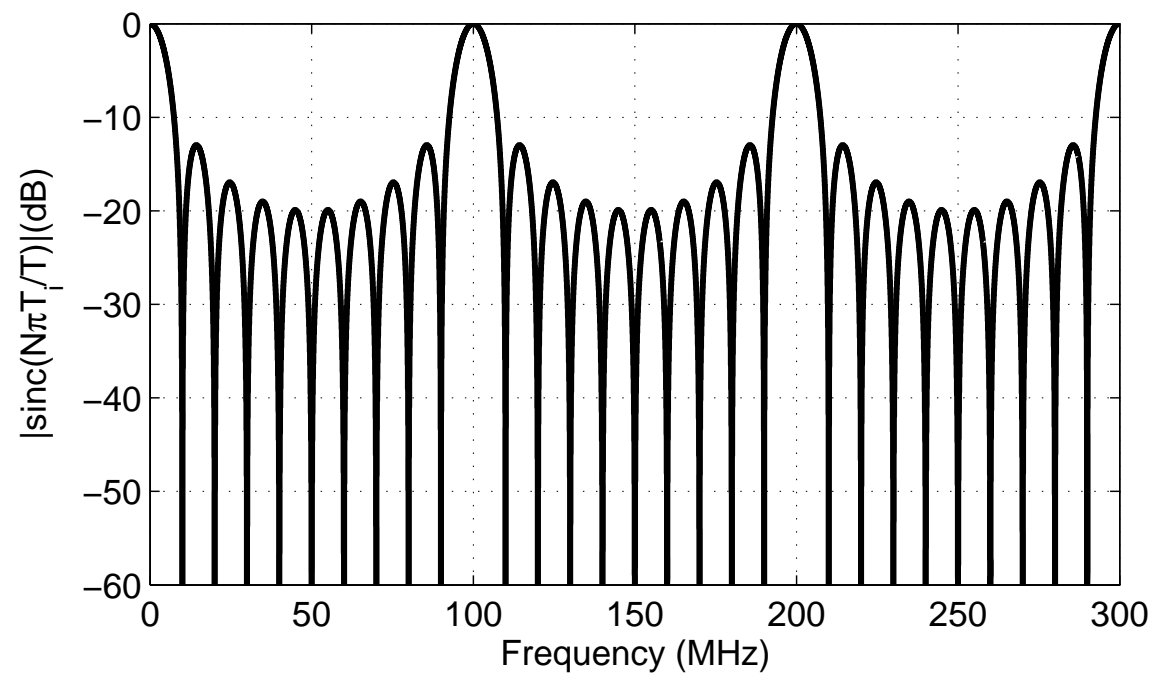

Figure 4.13: Spectrum of charge-domain windowed sampler with FIR filtering. Parameters: $f_{s}=10 \mathrm{MHz}, N=10$ and $T_{f}=T_{s} / N$.

In conclusion, the $\mathrm{CT}$ sinc low-pass response from the current windowed integration nevertheless produces zeros at the multiples of the frequency $1 / T_{i}$. The first sidelobe provides maximal $13 \mathrm{~dB}$ attenuation on unwanted signal. Like conventional DT filters, the passband of the embedded FIR filter from multiple windowed integration of the current signal in charge-domain sampling circuits is repeated at intervals of its sampling frequency, $1 / T_{f}$. 


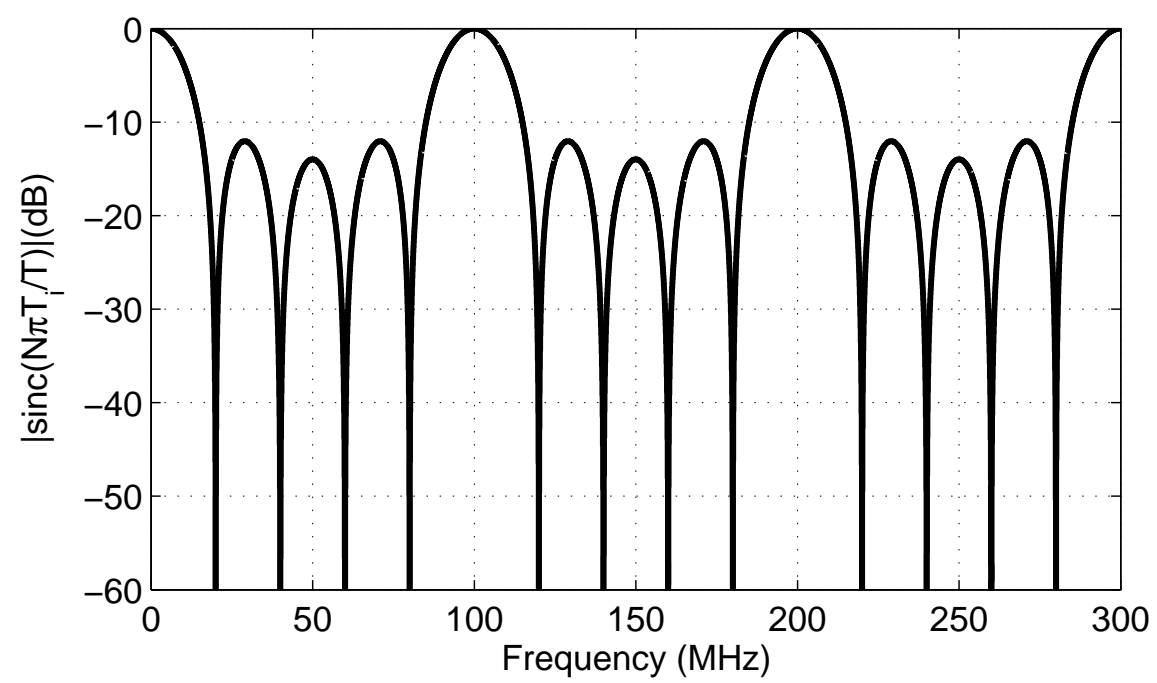

Figure 4.14: Spectrum of charge-domain windowed sampler with FIR filtering. Parameters: $f_{s}=20 \mathrm{MHz}, N=5$ and $T_{f}=T_{s} / N$.

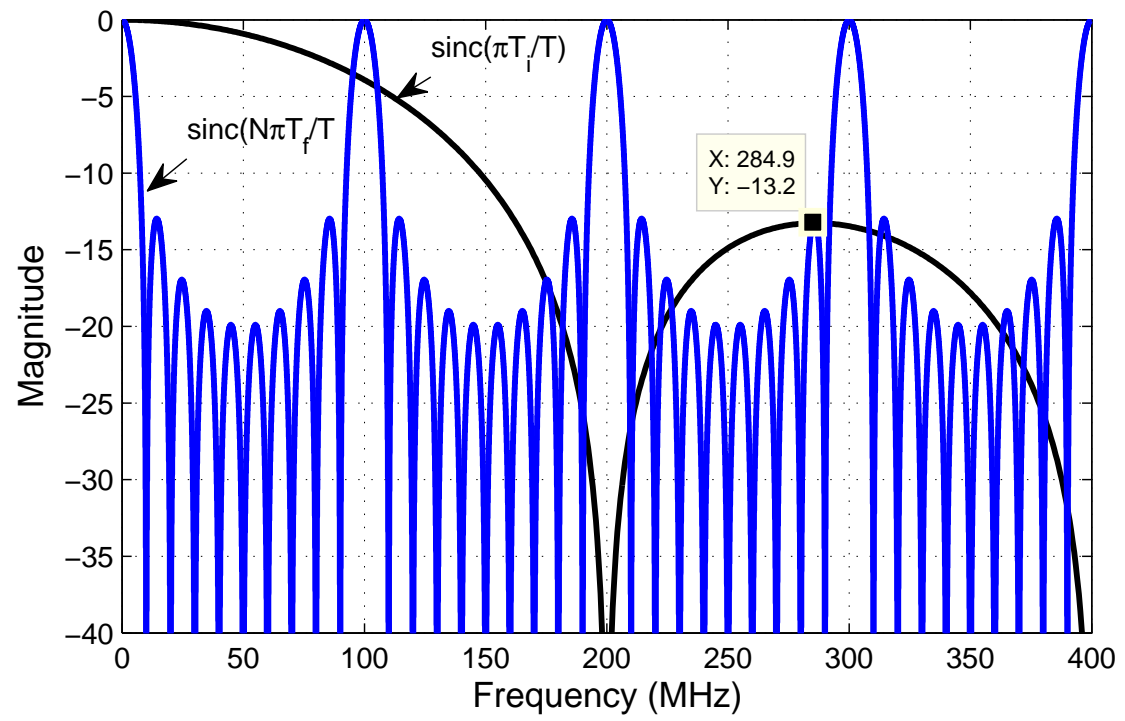

Figure 4.15: Spectrum of charge-domain windowed sampler with CT sinc low-pass filter. $f_{i}=200 \mathrm{MHz}, f_{s}=10 \mathrm{MHz}, N=10$ and $T_{f}=T_{s} / N$.

The final readout rate of the sampler is set at $T_{s}=N T_{f}$. In addition, the phase response of $\mathrm{CT}$ and DT sinc filters are ideally linear and can be denoted by group delay in time-domain response. 


\subsubsection{IIR Filter}

The build-in CT sinc and DT sinc low-pass filter of the charge-domain sampler developed in above limit the input bandwidth of the sampler and suppress the aliasing signal at the integer multiples of $1 / T_{i}$ and $N / T_{f}$, which effectively provide anti-aliasing filtering function for the sampler. It is worth to note that the selectivity for channel selection, however, is merely elementary and limited. High order or more advanced FIR filter can be implemented to improve the filtering functions, e.g. sharper frequency transition band, and larger attenuation in sidelobs. The cost is increased complexity of the circuit and power consumption. In comparison with FIR filter, a infinite impulse response (IIR) filter is efficient to provide same frequency response while consuming less arithmetic resources. It was shown in [80] a IIR low-pass characteristic can be obtained by employing the recursive operation of a history capacitor and rotating capacitors, as shown in Fig. 4.16. The time interleaved structure is shown in Fig. 4.17.
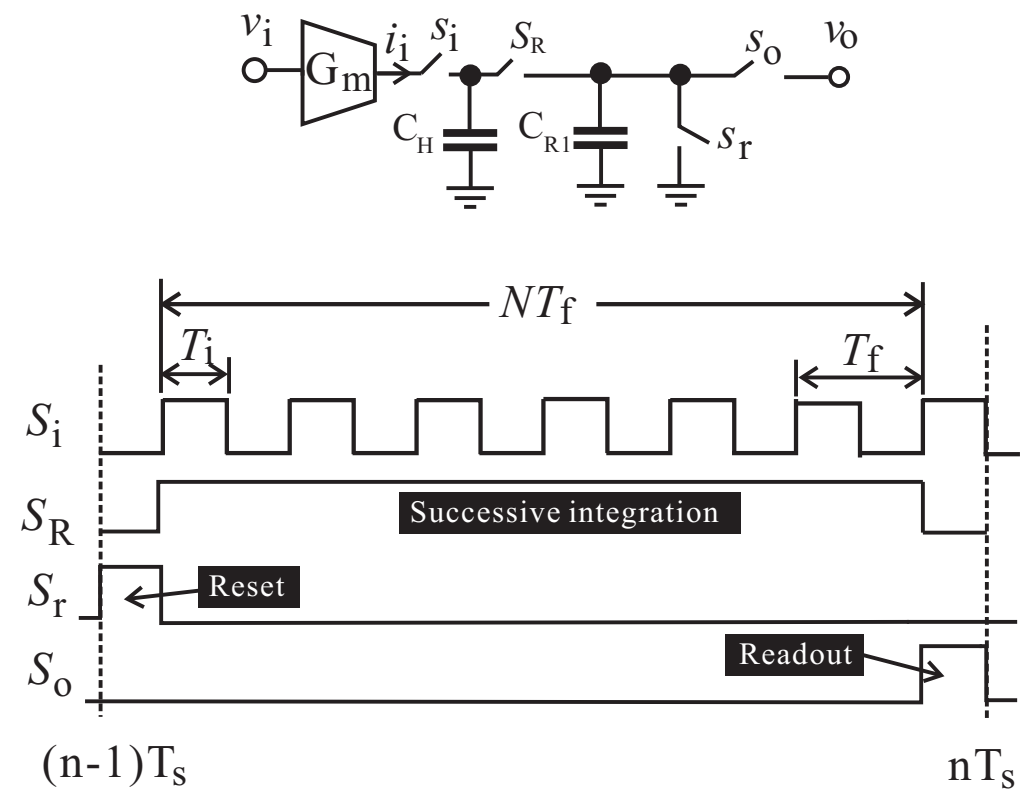

Figure 4.16: Charge-domain sampler with a build-in FIR and IIR filter and its timing scheme. 

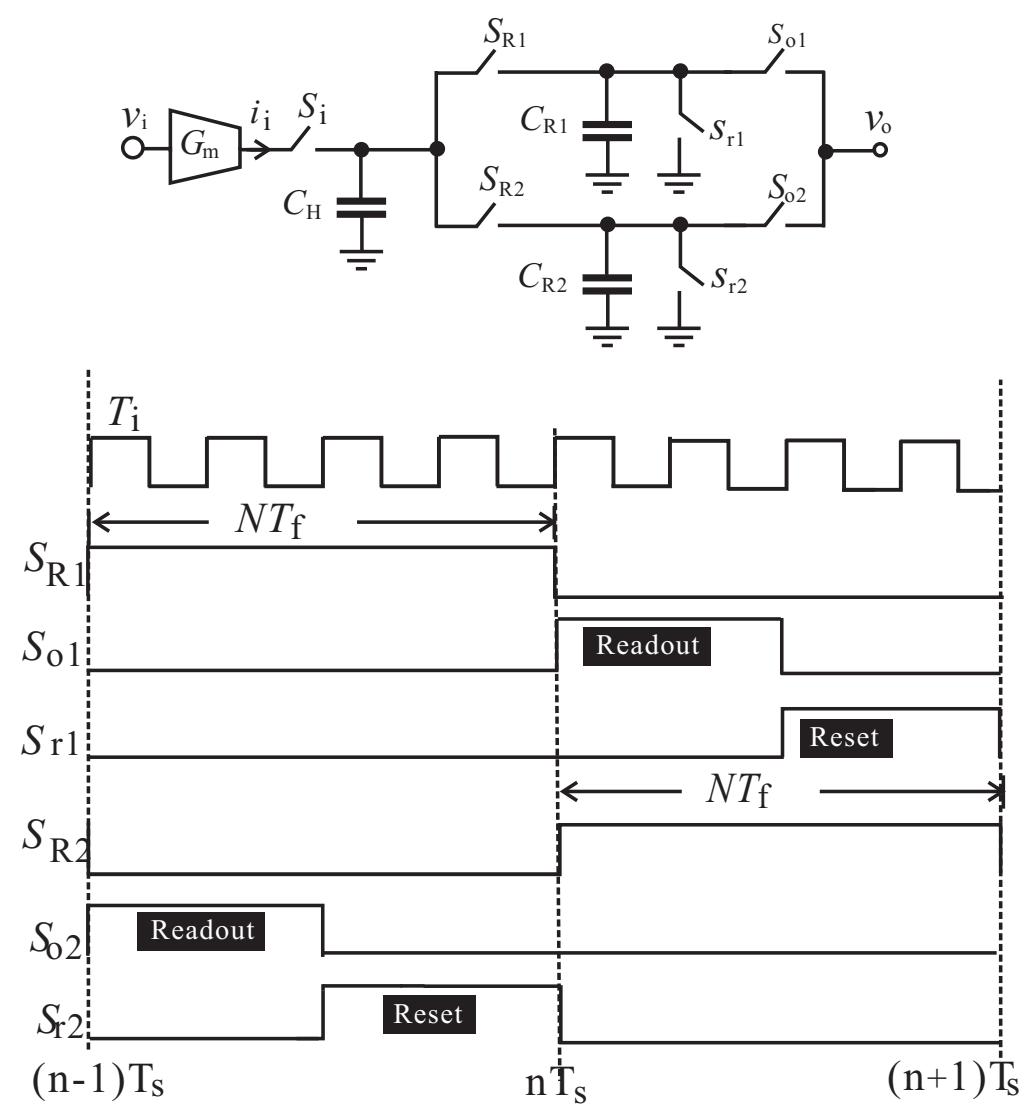

Figure 4.17: Time interleaved charge-domain sampler with a build-in FIR and IIR filter and its timing scheme.

From Fig. 4.17, the integration capacitor is partitioned into history capacitor $C_{H}$ and rotation capacitors $C_{R 1}$ and $C_{R 2} . C_{H}$ is always charged by the windowed current from the transconductor, and it is never discharged to entirely. While the rotation capacitors are sharing the charges with $C_{H}$ and discharging entirely. $C_{R 1,2}$ are switched between reset/integration and read-out operation. Assume $C_{H}=a C_{s}$ and $C_{R 1, R 2}=(1-a) C_{s}$, the total integration capacitance seen by $i_{i}(t)$ is always $C_{s}=C_{H}+C_{R 1, R 2}$. Consider $(n-1)$ th phase and assume $S_{R 1}=1$ and $S_{R 2}=0$. The current is integrated onto $C_{H}+C_{R 1}=C_{s}$ while the charge of $C_{R 2}$ is transferred to the load. Let the total charge stored in the integration capacitors $C_{H}$ and $C_{R 1}$ in $(n-1)$ th phase be $Q(n-1)$. In the following $n$th phase where $S_{R 1}=0$ and $S_{R 2}=1$, the charge stored in $C_{R 1}$ is transferred to the load. Since $C_{R 1}$ and $C_{R 2}$ are always reset before they are connected to $C_{H}$, the loss of the charge of the integration capacitors will be the charge of $C_{R 1}$. If the total charge stored in the integration capacitors 
$C_{H}$ and $C_{R 2}$ at the end of $n$th phase is $Q(n)$, the amount of the charge provided by the transconductor during $n$th phase, denoted by $Q_{i n}(n)$, is equal to the charge of $C_{R 1}$ and is therefore given by $Q(n)-a Q(n-1)$, i.e.

$$
Q(n)=a Q(n-1)+Q_{i n}(n)
$$

Or equivalently, it can be expressed in the z-domain as:

$$
\frac{Q_{z}}{\operatorname{Qin}}=\frac{1}{1-a z^{-1}}
$$

where $a=\frac{C_{H}}{C_{H}+C_{R 1, R 2}}$, a value which is always smaller than unity. Since $Q(n)=C_{s} V_{o, I I R}(n)$, $Q(n-1)=C_{s} V_{o, I I R}(n-1)$, and $Q_{i n}(n)=C_{s} V_{o, F I R}(n)$, note that the output of the FIR filter is the input of the IIR filter as

$$
V_{o, I I R}(n)=a V_{o, I I R}(n-1)+V_{o, F I R}(n)
$$

Eq.(4.21) is a first-order IIR filter. It can be shown from $z^{-1}=e^{s T_{s}} \approx 1-s T_{s}$ that

$$
H_{I I R}(s) \approx \frac{1}{1-a} \frac{1}{s\left(\frac{a T_{s}}{1-a}\right)-1} .
$$

The pole of the first-order IIR filter is given by $\omega_{p, I I R}=\frac{1}{T_{s}}\left(\frac{1}{a}-1\right)$ and the gain of the IIR filter is maximized at DC and is given by $1 /(1-a)$. It is seen that the larger $a$ is, the larger the gain of the IIR filter and the smaller the pole frequency subsequently the more the attenuation. In other words, $a$ plays a key role in input bandwidth and attenuation. 
This agrees well with the simulated results in Fig. 4.18, showing a frequency response of first-order IIR low-pass filter for different capacitance ratio values .

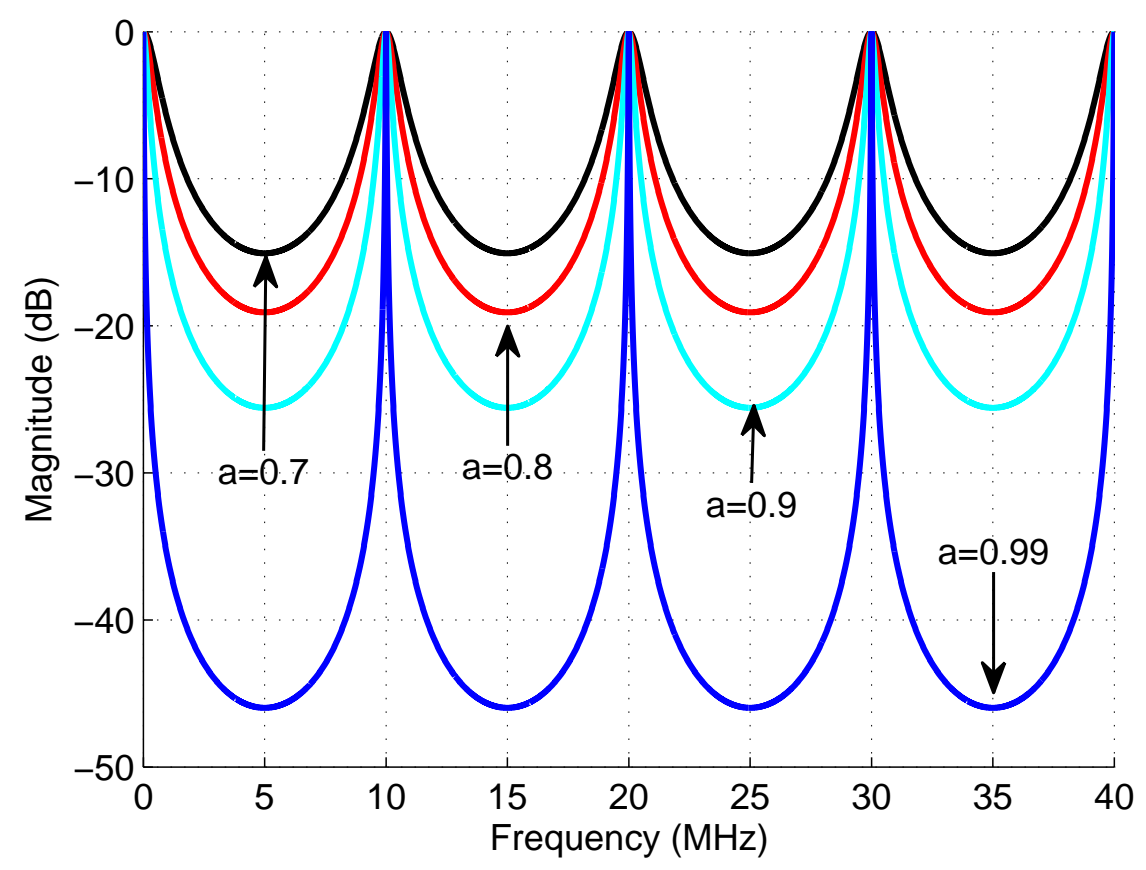

Figure 4.18: Spectrum of charge-domain windowed sampler with first-order IIR filter. $f_{s}=10$ $\mathrm{MHz}$.

In conclusion, a partial channel select filter is carried out by using first-order DT IIR low-pass filter in the charge-domain sampler with acceptable modification. The IIR filtering operation is taking place at the output sampling rate, $f_{s}=1 / T_{s}$. The pole location of the IIR filter is merely determined by the capacitor value ratio, $a=\frac{C_{H}}{C_{H}+C_{R 1, R 2}}$, which can be precisely controlled by layout, and $f_{s}$. The passbands of the IIR filter are repeated at intervals of $k f_{s}$. It is noticed that in Fig. 4.17, there are a total number of three filters taking place in the signal path, i.e. the first-order CT sinc filter, the first-order DT sinc filter, and the first-order DT integrator. 


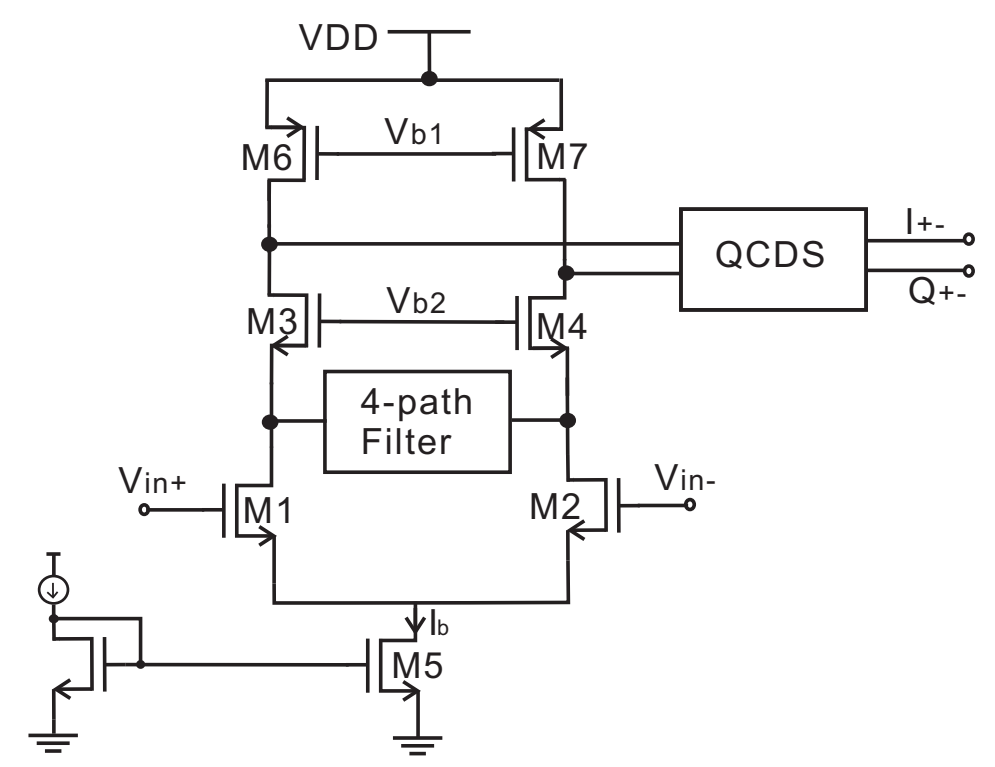

Figure 4.19: Quadrature Charge-Domain Sampler with 4-path bandpass filter. Circuit Parameters: $W_{1,2}=20 \mu \mathrm{m}, W_{3,4,5}=15 \mu \mathrm{m}, W_{6,7}=10 \mu \mathrm{m}, L=0.36 \mu \mathrm{m}, I_{b}=150 \mu \mathrm{A} ., V_{b 1}=0.67 \mathrm{~V}$, $V_{b 2}=0.65 \mathrm{~V}$.

\subsection{Circuit Design and Analysis}

The quadrature charge-domain sampler is implemented in a fully-differential configuration, shown in Fig. 4.19. The differential input voltage is converted to a current by the transconductor formed by M1 and M2. The signal then passes through the 4-path band-pass filter where aliasing interferences and unwanted signals are attenuated. Transistors M3/M4 form a current buffer with a low input resistance $1 / g_{m 3,4}$. Transistors M3/M4 form a current buffer with a low input resistance $1 / g_{m 3,4}$. A large output resistance at the drain of M3/M4 and M6/M7 can be obtained only in the band of the 4-path filter. In other words, the resistance exhibits a relatively low value at out of the band of the 4-path filter. The schematic of differential 4-path filter is shown in Fig. 4.20 .

As differential structure, the selectivity of the band-pass filter at the even harmonics of the clock is suppressed. Each path is includes one grounded capacitor connected to two anti-phase driven switches. A 4-phase 25\% duty cycle clock provides all required clocks. The switches are implemented by using transmission gate (TG) with large size to minimize the ON-resistance, which affects the profile of the band-pass frequency response. On the 


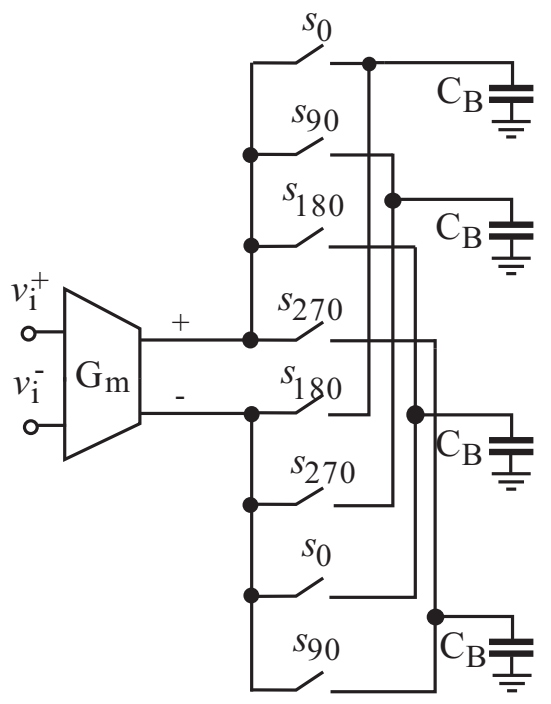

(a)

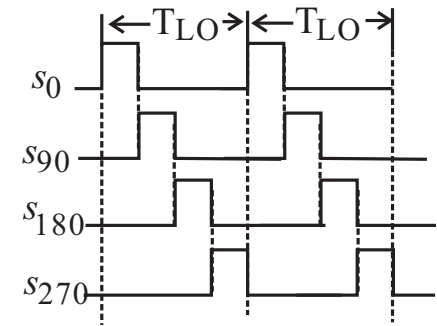

(b)

Figure 4.20: (a) Differential 4-path band-pass filter, (b) Clocking scheme.

other hand, the large parasitic capacitors because of the large TG can be merged into the capacitance of base-band capacitors, $C_{B}$. The PSS-PAC simulated result is shown in Fig. 4.21 .

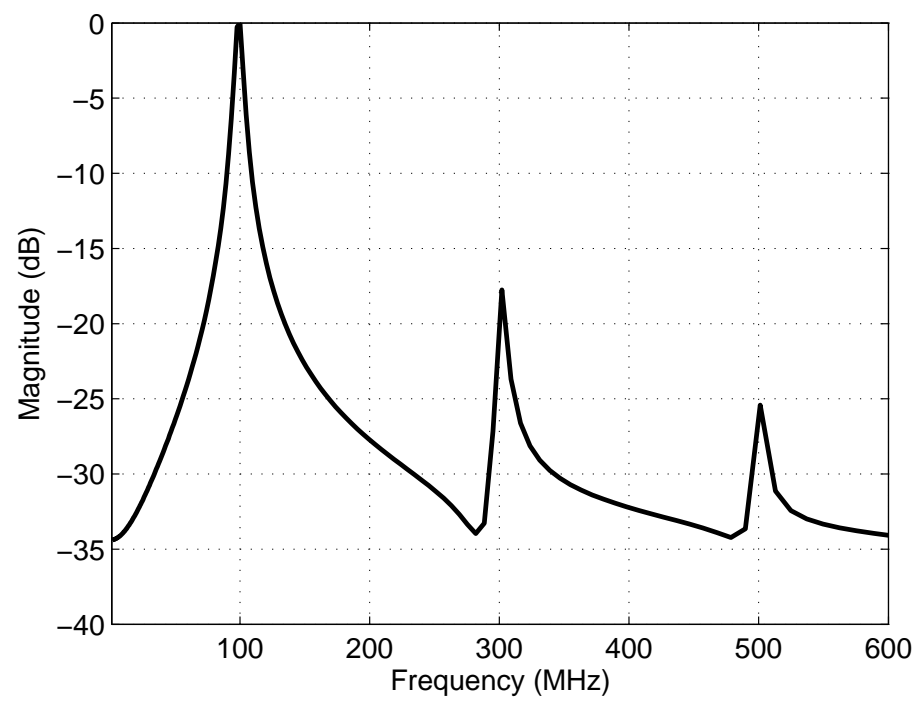

Figure 4.21: Simulated differential 4-path band-pass filter with $T_{s}=T_{L O}=100 \mathrm{MHz}$. 
A complete circuit schematic of I channel of quadrature charge-domain sampler (QCDS) is shown in Fig. 4.22. The schematic for Q channel is identical except that FLO90 and FLO270 are used in Q channel. The QCDS is fully differential, and for each of I and Q channels, the signal path is further split into two paths as time interleaved configuration. Since both the 4-path band-pass filter and quadrature charge-domain sampler contain only capacitors and switches, and the currents of the transconductor M1/M2 are re-used by the quadrature charge-domain sampler, minimizing power consumption.

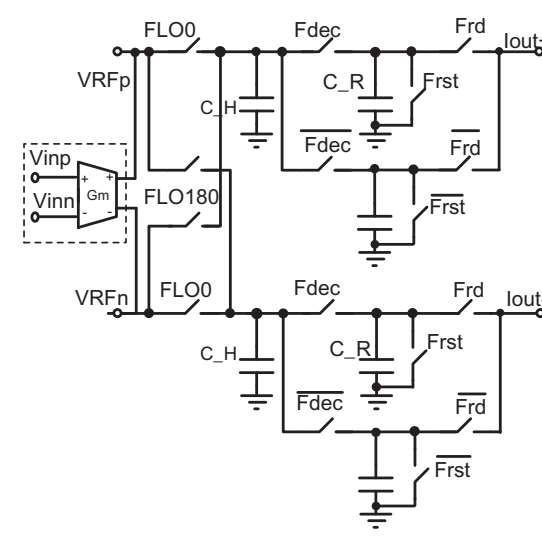

(a)

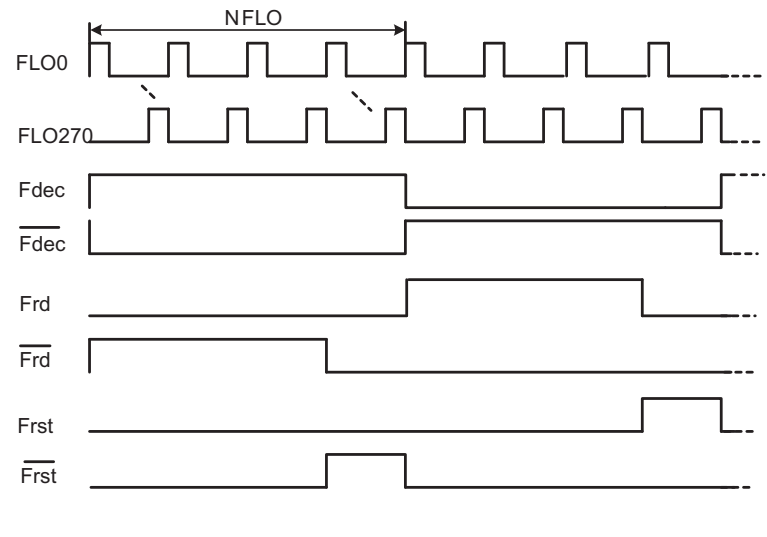

(b)

Figure 4.22: (a) Differential charge-domain sampler (only half of the circuit is shown), (b) Clocking scheme.

\subsubsection{Performance}

The entire system consists of four distinct functional blocks, namely, a 4-path bandpass filter, a continuous-time sinc low-pass filter, a FIR filter, and an IIR low-pass filter with transfer function $H_{B P}, H_{\text {sinc }}, H_{F I R}$, and $H_{I I R}$, respectively. The overall transfer function is given by $H=H_{B P} H_{s i n c} H_{F I R} H_{I I R}$. The 4-path bandpass $H_{B P}$ provides a maximum of $-40 /$ dec dB attenuation. The continuous-time sinc low-pass obtained from the continuous integration of the input over $T_{i}$ provides $-20 / \mathrm{dec} \mathrm{dB}$ attenuation. The IIR filter provides another $-20 /$ dec $\mathrm{dB}$ attenuation. Finally, the attenuation provided by the FIR filter is determined by the number of integration phases and the weight factors. Although the overall 
attenuation is large, since $-3 \mathrm{~dB}$ frequency of these filters differ and it is likely not possible to have all the filters to have the same $-3 \mathrm{~dB}$ frequency, the attenuation in the desired stop-band is rather small. The nonidealities of devices further reduces the attenuation. Fig. 4.23 shows the output of the quadrature charge-domain sampler with different history capacitor. It can be seen that increasing the capacitance of the history capacitor improves the attenuation in the stop band and the depth of the nulls. Attenuation is further increased when the 4-path bandpass filter is added. Also, the depth of the nulls of the sampler with the 4-path bandpass filter are much deeper as compared with that without the 4-path bandpass filter. This observation reveals that the 4-path bandpass filter not only attenuates the tones in the stop-band, qualifying it as a band select filter to suppress blockers, it also improves the suppression of aliasing interferences.

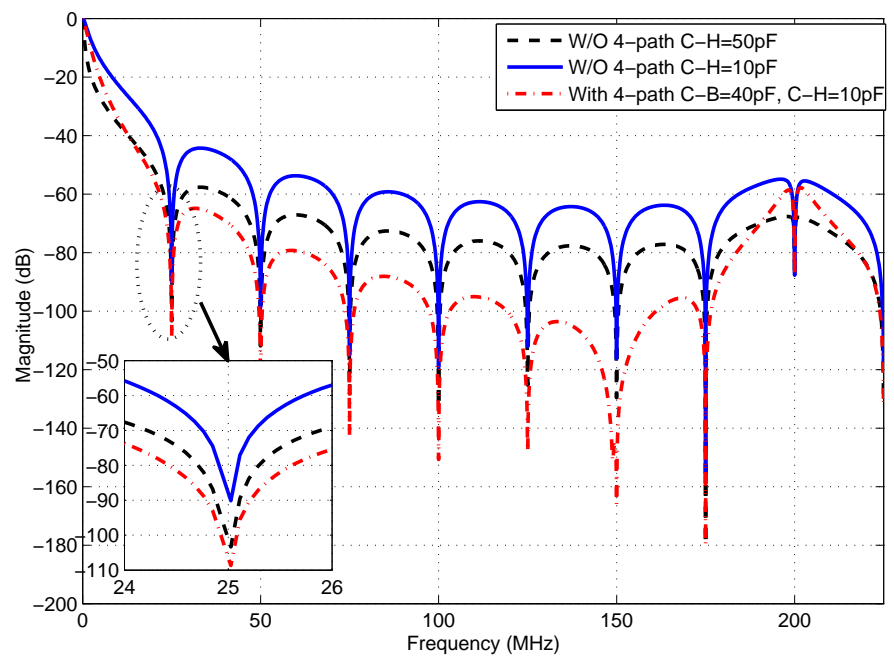

Figure 4.23: Simulated transfer function (normalized) of quadrature charge-domain sampler. Ideal transconductor with $g_{m}=0.5 \mathrm{mS}$ and ideal switches with $R_{o n}=1 \Omega$ and $R_{o f f}=1 \mathrm{~T} \Omega$ are used. The input is a $100 \mathrm{MHz}$ sinusoid. The sampling frequency is $100 \mathrm{MHz}$ with $25 \%$ duty cycle. Voltage gain provided by the sampler is $15 \mathrm{~dB}$. 


\subsubsection{Noise}

An explicit noise analysis of the quadrature charge-domain sampling circuit is crucial as the noise contribution to the total system Noise Figure can be significant due to the limited gain of the preceding LNA. In order to evaluate the noise performance of the circuit, follow the approach in [113]. Let the power of the input-referred noise-voltage generator of the sampling mixer be $\overline{v^{2}}{ }_{n}$. The power of input-referred noise-current generator of the sampling mixer is negligible. The total input noise is obtained from ${\overline{v^{2}}}_{n, i n}={\overline{v^{2}}}_{n, R_{s}}+{\overline{v^{2}}}_{n}$ where $\bar{v}_{n, R_{s}}$ is the noise power of the source resistor.

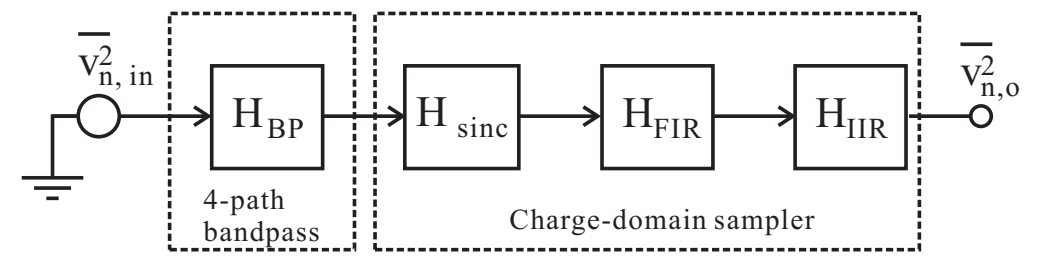

Figure 4.24: Noise analysis of proposed charge-domain sampling mixer.

From the schematic of the sampling mixer, except for the noise voltage from source, there are two noise sources existing, e.g. modelled thermal noise of output impedance of the transconductor and the thermal noise of switch resistors. Compared with output impedance of transconductor, $R_{\text {on }}$ is typically small. For example, in Fig. $4.20, R_{\text {out }} \approx 10 \mathrm{k} \Omega$ and $R_{\text {on }}=15$. Since $R_{\text {out }} \gg R_{\text {on }}$, the noise voltage of switch resistors is significantly smaller than that of $R_{\text {out }}$. Therefore, the noise voltage of switch resistors can be ignored and only modelled noise voltage of output impedance is related to $\bar{v}_{n}{ }_{n}$. As shown in Fig. 4.24, the input noise is first shaped by the 4-path bandpass filter. It then passes through the charge-domain sampler that provides a continuous-time first-order sinc low-pass $H_{\text {sinc }}(j \omega)$, a discrete-time FIR filter $H_{F I R}(j \omega)$ from the multiple sub-integration phases, and the first-order IIR filter $H_{I I R}(j \omega)$ from the history and rotation capacitors. The total power of the output noise is obtained from 


$$
\overline{v_{o}^{2}}=\int_{0}^{\infty}\left|H_{B P} H_{s i n c} H_{F I R} H_{I I R}\right|^{2} \bar{v}_{n, i n} d \omega .
$$

To quantify the effect of $H_{B P}, H_{\text {sinc }}, H_{F I R}$, and $H_{I I R}$, the noise bandwidth of the sampling mixer with $H_{B P}, H_{\text {sinc }}, H_{F I R}$, and $H_{I I R}$ are considered individually. To simplify analysis, assume ${\overline{v^{2}}}_{n, \text { in }}$ is white, and the noise of interferences is not taken into consider.

\section{Noise Bandwidth With $H_{B P}$ Considered}

Consider the effect of the N-path bandpass only. For the 4-path filter shown in Fig. 4.20, the baseband block is a first-order low-pass with transfer function $H_{B B}=1 /\left(s / \omega_{B}+\right.$ 1) where $\omega_{B}=1 /\left(R_{\text {out }} C_{B}\right)$ is $-3 \mathrm{~dB}$ frequency and $R_{\text {out }}$ is the output impedance of the transconductor. The transfer function of the N-path band-pass filter was derived earlier in (4.12), where the thermal noise, attenuated by the periodical band-pass filtering at the multiple $\omega_{s}$ and is folded back to the desired signal. In the design, $R_{\text {out }} \approx 10 \mathrm{k} \Omega$ and $C_{B}=40$ $\mathrm{pF},-3 \mathrm{~dB}$ frequency is $398 \mathrm{KHz}$ while the sampling frequency is $100 \mathrm{MHz}$ and the first notch is located $400 \mathrm{MHz}$. As a result, the input noise voltage is folded at $300 \mathrm{MHz}$ with $20 \mathrm{~dB}$ attenuation, $30 \mathrm{~dB}$ attenuation at $500 \mathrm{MHz}$ and etc. This is evident in (4.24) where output noise power of the 4-path filter at $\omega-\omega_{s}$ is computed and illustrated graphically in Fig. 4.25 .

$$
\begin{aligned}
\overline{v_{n, o}^{2}}\left(\omega-\omega_{s}\right) & =0.0625\left[\left|H_{B B}\left(\omega-\omega_{s}\right)\right|^{2}+\operatorname{sinc}^{4}\left(\frac{\pi}{4}\right)\left|H_{B B}(\omega)\right|^{2}\right. \\
& \left.+\operatorname{sinc}^{4}\left(\frac{2 \pi}{4}\right)\left|H_{B B}\left(\omega+\omega_{s}\right)\right|^{2}+\ldots\right]{\overline{v^{2}}}_{n, i n} .
\end{aligned}
$$

Since $\operatorname{sinc}^{4}\left(\frac{\pi}{4}\right) \approx 0.656, \operatorname{sinc}^{4}\left(\frac{\pi}{2}\right) \approx 0.16, \operatorname{sinc}^{4}\left(\frac{3 \pi}{4}\right) \approx 0.009, \operatorname{sinc}^{4}\left(\frac{5 \pi}{4}\right) \approx 0.001, \operatorname{sinc}^{4}\left(\frac{6 \pi}{4}\right) \approx 0.002$, $\operatorname{sinc}^{4}\left(\frac{7 \pi}{4}\right) \approx 0.0003, \ldots$, the folding effect can be approximated by only considering the first two terms of (4.24). As a result, the output noise power at $\omega-\omega_{s}$ is given by $\overline{v_{n, o}^{2}}\left(\omega-\omega_{s}\right)=$ $0.074 \bar{v}_{n, i n}$. 


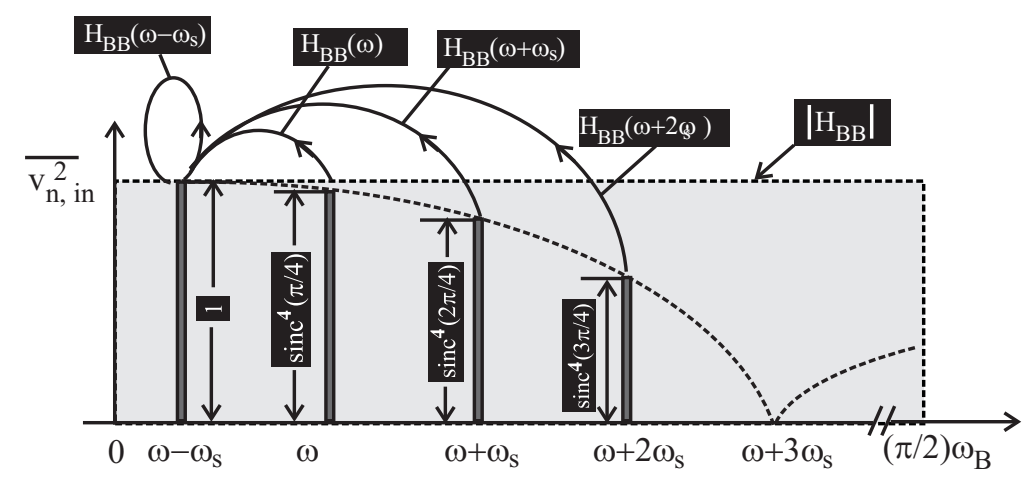

Figure 4.25: Noise analysis of N-path filter.

The noise bandwidth of the baseband block is given by $\frac{\pi}{2} \omega_{B}$. To find out the noise bandwidth of the sampling mixer with only $H_{B P}$ considered, the calculated total output noise power of the sampling mixer is given by

$$
\begin{aligned}
\overline{v_{n o}^{2}} & =\int_{0}^{\infty}\left|H_{B P}\right|^{2} \overline{v_{n, i n}^{2}} d \omega \\
& =\left(\frac{1}{N^{2}}\right)^{2} \sum_{n=-\infty}^{\infty} \operatorname{sinc}^{4}\left(\frac{n \pi}{N}\right)\left(\frac{\pi}{2} \omega_{B}\right) \bar{v}_{n, i n} \\
& \approx 0.074\left(\frac{\pi}{2} \omega_{B}\right) \bar{v}_{n, i n} .
\end{aligned}
$$

Note $N=4$ was utilized in (4.25).

\section{Noise Bandwidth With $H_{s i n c}$ Considered}

Consider the effect of $H_{\text {sinc }}$ only. Utilizing (4.14), we obtain from (4.23)

$$
\overline{v_{o}^{2}}=\frac{1}{2 \pi}\left(\frac{G_{m} T_{i}}{C_{s}}\right)^{2} \int_{0}^{\infty} \operatorname{sinc}^{2}\left(\frac{\omega T_{i}}{2}\right) \bar{v}_{n, i n} d \omega
$$

Since $\int_{0}^{\infty} \operatorname{sinc}^{2}(x) d x=\frac{\pi}{2}$, Eq. (4.26) becomes $\overline{v_{o}^{2}}=\frac{G_{m}^{2}}{C_{s}^{2}} \frac{T_{i}}{2} \bar{v}_{n, i n}$. Since $\overline{v_{o}^{2}}=\frac{G_{m}^{2} T_{i}^{2}}{C_{s}^{2}} \bar{v}_{n, i n} \omega_{N, \operatorname{sinc}}$ where $\omega_{N, \text { sinc }}$ is the noise bandwidth when only $H_{\text {sinc }}$ is considered, we have $\omega_{N, \text { sinc }}=\frac{\omega_{i}}{2}=\omega_{f}$ where $\omega_{i}=2 \pi / T_{i}$. 


\section{Noise Bandwidth With $H_{F I R}$ Considered}

Consider the effect of $H_{F I R}$ only. Utilizing (4.17), we have from (4.23)

$$
\overline{v_{o}^{2}}=\int_{0}^{\infty} \operatorname{sinc}^{2}\left(N \pi T_{f} f\right){\overline{v^{2}}}_{n, i n} d f=\frac{N}{2 T_{f}} \bar{v}_{n, i n} .
$$

Since

$$
\overline{v_{o}^{2}}=N \bar{v}_{n, i n} \omega_{N, F I R}
$$

where $\omega_{N, F I R}$ is the noise bandwidth when only $H_{F I R}$ is considered, we have $\omega_{N, F I R}=\frac{\omega_{f}}{2}$ where $\omega_{f}=2 \pi / T_{f}$.

\section{Noise Bandwidth With $H_{I I R}$ Considered}

Consider the effect of $H_{I I R}$ only. Since $H_{I I R}$ is a first-order low-pass with pole frequency $\omega_{I I R}=\frac{1}{T_{s}}\left(\frac{1}{a}-1\right)$, the noise bandwidth due to $H_{I I R}$ is given by $\omega_{N, I I R}=\frac{\pi}{2} \omega_{I I R}$. Since $T_{s}=T_{r}+T_{o}+N T_{f}$, if $N T_{f} \gg T_{r}, T_{o}$, we have $T_{s} \approx N T_{f}$. As a result, $\omega_{N, I I R} \approx \frac{1}{4 N}\left(\frac{1}{a}-1\right) \omega_{f}$.

Table 4.1: Noise Bandwidth of Sampling Mixer with 4-path Bandpass, sinc, FIR, and IIR Filters Considered Individually.

\begin{tabular}{ll}
\hline Filter & Noise bandwidth \\
\hline N-path bandpass & $\omega_{N, B P}=\pi / 2 \omega_{B}$ \\
sinc low-pass & $\omega_{N, \text { sinc }}=\omega_{f}, \omega_{f}=\omega_{i} / 2$ \\
FIR filter & $\omega_{N, \text { FIR }}=\omega_{f} / 2, \omega_{f}=\omega_{i} / N$ \\
IIR filter & $\omega_{N, I I R}=1 / 4 N(1 / a-1) \omega_{f}$ \\
\hline
\end{tabular}

Table 4.1 tabulates the noise bandwidth of the sampling mixer with the 4-path bandpass, sinc, FIR, and IIR filters considered individually. In the design, $N=4, C_{H}=10 \mathrm{pF}$, $C_{R}=1 \mathrm{pF}$. Thus, $a=0.9$ and $\omega_{I I R}=\omega_{f} /(16 \times 9) \ll \omega_{N, F I R}, \omega_{N, \text { sinc }}$. Compared the noise bandwidth of four, $\omega_{N, B P}$ and $\omega_{N, I I R}$ is much smaller than the other two. The total output noise of 
the sampling mixer can therefore be obtained from ${\overline{v^{2}}}_{n o} \approx \omega_{N, I I R}{\overline{v^{2}}}_{n, i n}$ and ${\overline{v^{2}}}_{n o} \approx \omega_{N, B P}{\overline{v^{2}}}_{n, i n}$

\subsubsection{Effect of Nonidealities}

Fig. 4.26 investigates the effect of the ON-resistance of the switches on the performance of the 4th-path band-bass filter. It is seen that the larger the resistance of the switches, the less the attenuation in the stop band, revealing that minimizing the channel resistance of the switches is essential.

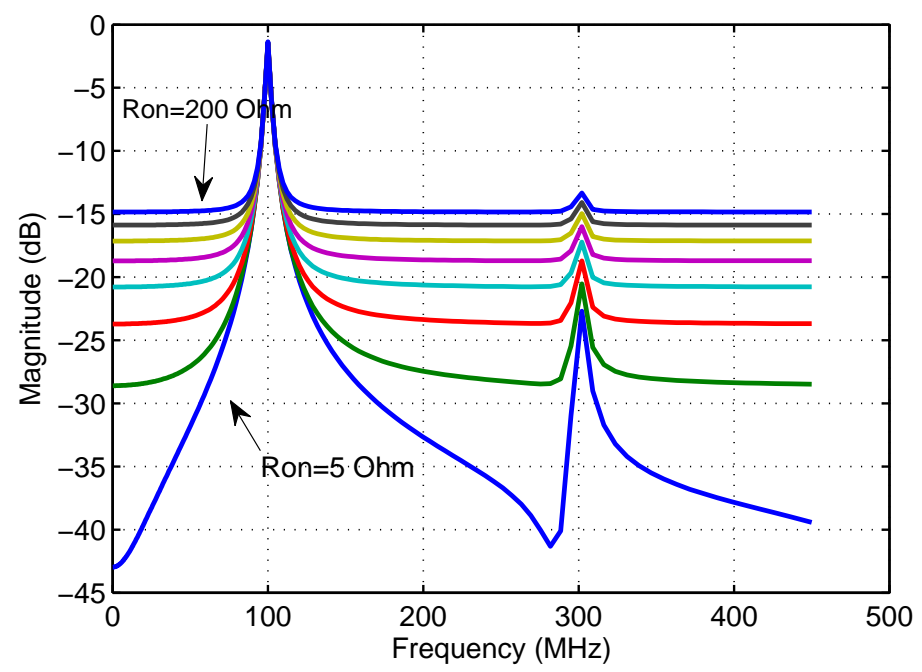

Figure 4.26: Simulated effect of ON-resistance of switches on 4-path bandpass filter. $R_{o n}$ is varied from $5 \Omega$ to $200 \Omega$.

Since the parasitic capacitances of switches are absorbed into the large sampling capacitors $(40 \mathrm{pF})$ of the 4-path bandpass filter, their effect on the performance of the filter is rather small. The parasitic capacitances of the switches, however, lower the gain of the charge-domain sampler. Unlike the 4-path bandpass filter, the ON-resistance of switches has a less detrimental effect on the charge-domain sampler [86]. To maximize the gain, small switches are used in QCDS. Fig. 4.27 shows the normalized transfer function of the quadrature charge-domain sampling receiver with embedded FIR, IIR, and 4-path bandpass 
filters at process corners. It is observed that the variation of attenuation due to process spread is within $10 \mathrm{~dB}$. The voltage gain is $6.9 \mathrm{~dB}$ for slow nMOS/slow pMOS (SS) and 14.4 $\mathrm{dB}$ for fast nMOS/fast pMOS (FF).

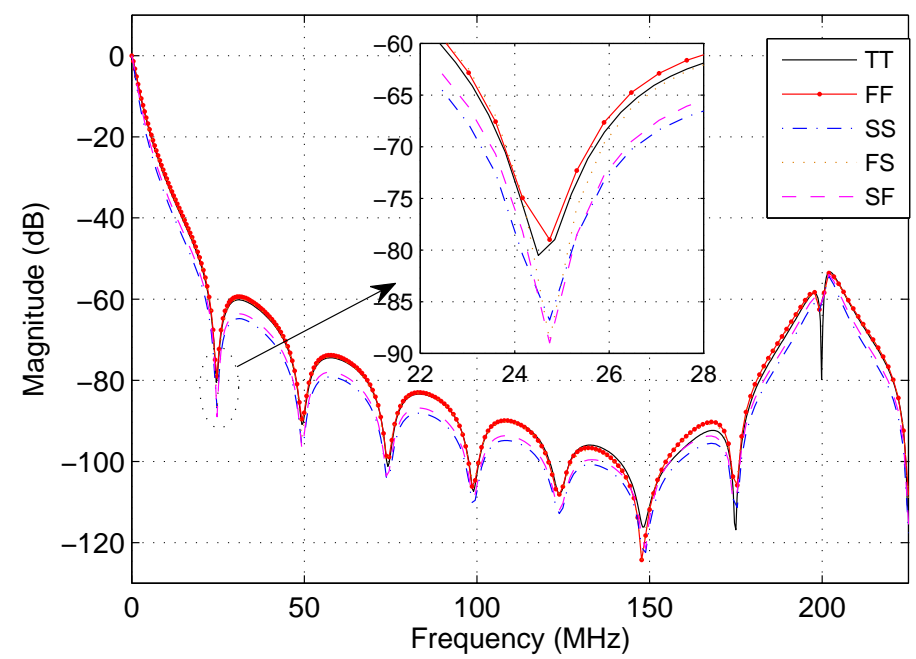

Figure 4.27: Simulated frequency response (normalized) of quadrature charge-domain sampler with embedded 4-path bandpass filter at process corners. The input frequency is $100 \mathrm{MHz}$, the output baseband sampling frequency is $25 \mathrm{MHz}$ (Two $12.5 \mathrm{MHz}$ interleaved clocks with $50 \%$ duty-cycle). Legends: TT (typical nMOS/typical pMOS), SS (slow nMOS/slow pMOS), FS (fast nMOS/slow pMOS), SF (slow nMOS/fast pMOS), and FF (fast nMOS/fast pMOS).

Not like simple sampling systems, the effect of the non-ideal clock is more clear. Since the design is a multiple sampling system, the impacts from sampling clocks are complex but critical.

clock phase noise - To simply analysis, let us only consider the desired signal frequency, $f_{\text {in }}$. For 4-path filter, if ideal sampling clocks, $f_{s}$, are applied to each of paths, only frequency component located at $f_{\text {in }}$ will be first translated into the baseband and integrated on the capacitor. The capacitor is acting as a low-pass filter to short any unwanted signal out of filter bandwidth to the ground. On the contrary, the signal nearby DC will be held on the capacitor and then be translated back to the original frequency because of the second time ON-OFF of the switches. However, if the sampling clock is non-ideal, a skirt profile in spectrum, all the frequency components inside the skirt will be down-converted to baseband 
except for the desired signal. The baseband signal then will be up-converted to the desired frequency again where the unwanted components are added on top of the desired signal, deteriorating SNR. The worse case is there is large blocker signal falling in the skirt. This blocker will lower the SNR significantly as it will be added into the desired signal and the low-pass filter in the baseband fails to filter it out. As a result, the phase noise results in increasing noise figure. Similarly, in the charge-domain sampling circuit, phase noise of sampling clock has a detriment on the overall SNR. However, since the frequency translation is accompanied by a continuous-time and a discrete-time since low-pass anti-aliasing filter, which create notches at the sampling frequency, these notches will greatly suppress the effects from the clock phase noise. Meanwhile, the noise on the clock in time domain is denoted by jitter. The clock jitter will change the integration time for a charge-domain sampler, causing error in the final output voltage. While, the clock jitter will has less detrimental to the SNR in a certain frequency range in comparison with voltage sampler. The range is heavily depending on the integration time, $T_{i}$.

clock phase imbalance - There are two cases for N-path filter. Consider the variation of the duty cycle, if duty cycle is less than 25\%(4-path) for each of path, as we can expect the attenuation on the harmonics will be less. The notches associated with the number of the path will be shifted to higher frequency. Therefore, the harmonics, which are still at the same location, will get less attenuation. As a result, higher fold-over components will be added on the desired signal, deteriorating SNR. For the case of duty cycle is more than $25 \%$, charge sharing between each of the baseband capacitor is occurred. This charge sharing will significantly degrade the filtering function. Take the extreme case for example, duty cycle is $100 \%$, at the same moment, all the capacitors in the 4-path filter are sharing the same incoming charges. The characteristics of the N-path network will be entirely disappeared. The more overlapping, the more filtering function will be lost. In a conclusion, the case of more than $25 \%$ duty cycle is even worse than the case of less than $25 \%$. In addition, the imbalanced phase between each of the paths could lead to less image rejection and harmonic folding. For the charge-domain sampler, the clock phase imbalance could result in mismatch in the time-interleaved configuration and subsequently introduce pattern noise located at $f_{s} / 4, f_{s} / 2 \ldots$. which are highly undesirable. 
The frequency tuning of the sampling mixer is shown in Fig. 4.28. For each input frequency $\omega_{i}$, the sampling frequency $\omega_{s}$ is adjusted such that the output frequency of the sampling mixer remains unchanged. Since $T_{s}=T_{r}+T_{o}+N T_{i}, T_{i}$ will also change. Thus, when $\omega_{i}$ increases, $\omega_{s}$ will increase, resulting in a reduction in $T_{i}$. Since the gain of the charge-domain sampler at DC is given by $G_{m} T_{i} / C_{s}$, the gain will drop when $\omega_{i}$ increases. This agrees well with the simulation results in Fig. 4.28.

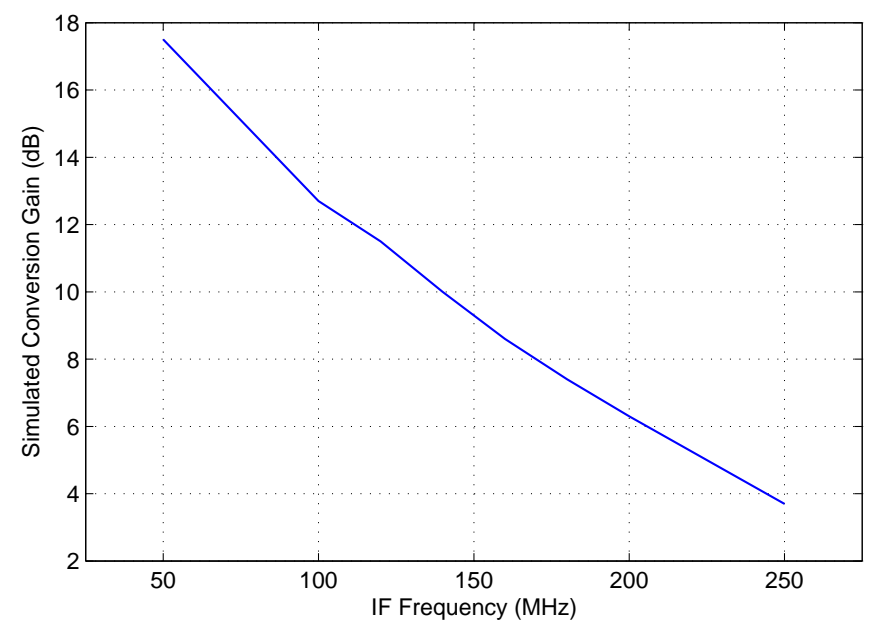

Figure 4.28: Simulated frequency tuning range. The input frequency is varied from $50 \mathrm{MHz}$ to $250 \mathrm{MHz}$. The sampling frequency is also varied accordingly such that the output frequency remains unchanged.

\subsection{Measurement Results}

The quadrature charge-domain sampler with embedded FIR, IIR, and 4-path bandpass filters was implemented in IBM cmrf8sf $130 \mathrm{~nm} \mathrm{1.2} \mathrm{V} \mathrm{CMOS} \mathrm{technology.} \mathrm{Fig.} 4.31$ shows the layout of the circuit. The entire chip is $1 \times 2 \mathrm{~mm}^{2}$. The capacitors in the 4-path filter are two-layer MIM capacitors that provide a higher capacitance density as compared with normal MIM capacitors. Capacitors in the charge-domain sampler are normal MIM capacitors that use only one mask between two metal layers. In addition, to provide an enough driving capability for the switches in the filter blocks, the clock output buffers were 
designed to drive a $200 \mathrm{fF}$ load capacitance using D flip-flops (DFFs) implemented using TSPC (True-Single-Phase Clocked) logic. The single-ended input signal from the RF signal generator is fed to the active balun shown in Fig. 4.30 where the single-ended input is converted to a differential input $\left(v_{i n}\right.$ to $v_{o}^{+}$via a non-inverting common-gate amplifier and $v_{\text {in }}$ to $v_{o}^{-}$via an inverting common-source amplifier). The balance of the gain of the two halves of the active balun can be obtained by adjusting $V_{b}$ of the common-gate. The differential signal is then fed to the transconductor of the 4-path filter. The output of the sampling mixer is fed to a source-follower buffer in order to drive an off-chip RF spectrum analyzer with a $50 \Omega$ input impedance.

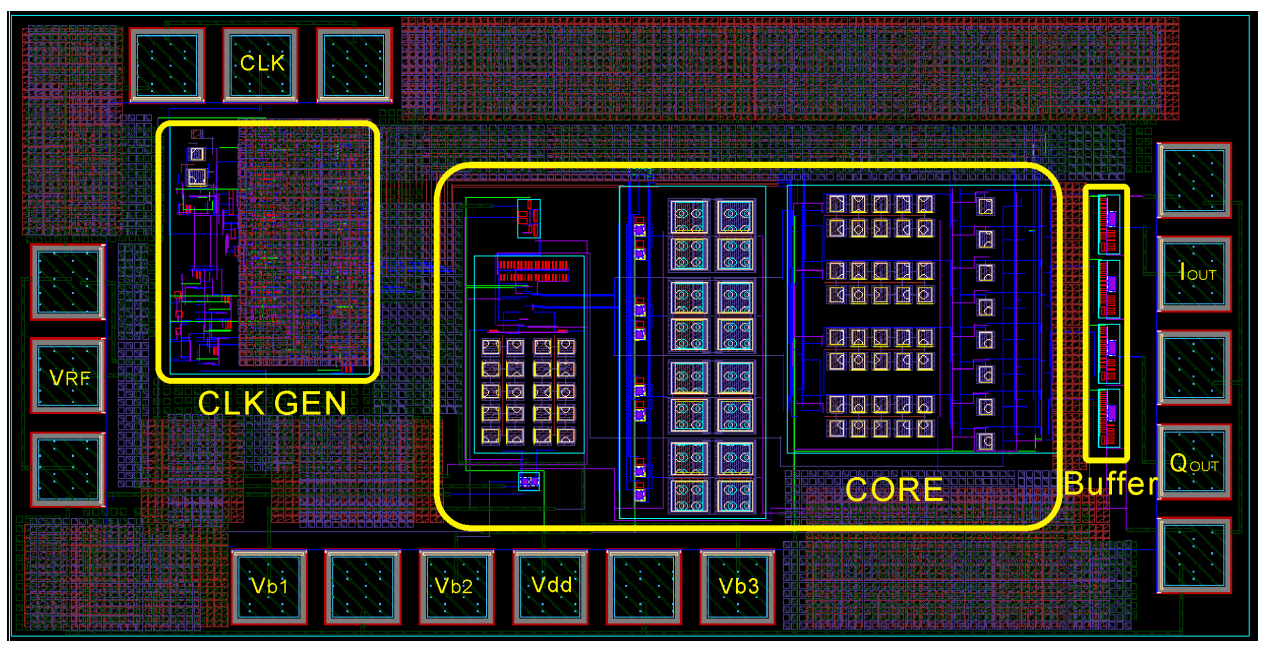

Figure 4.29: Die microphotograph of quadrature charge-domain sampler $\left(1 \mathrm{x} 2 \mathrm{~mm}^{2}\right)$.

Fig. 4.31 shows the photo-micrograph of the fabricated chip, with probes in place for on-wafer measurement. On-wafer probing was conducted using a Microtech RF-1 microwave probe station (with a Faraday cage) to minimize the effect of package parasitics and external electromagnetic interferers. The pads were arranged based on the configuration of the probes and available silicon area. A Microtech DCQ-06 dc probe, with six ceramic bladed needles configured as SGSSGS ( $\mathrm{S}=$ signal and $\mathrm{G}=$ ground), was used to supply the required de bias voltages to the chip. Two, single-ended, Microtech ACP-40-D-GSG-150 RF probes were used for the clock and input. One differential Microtech ACP-40-D-GSGSG-150 RF probe 


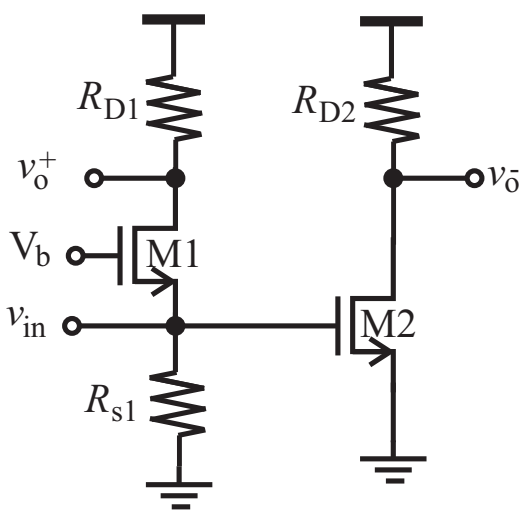

(a)

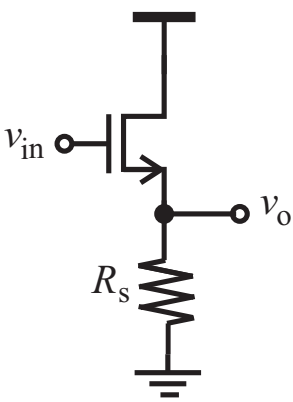

(b)

Figure 4.30: (a) Active balun. (b) Output buffer. Circuit parameters of active balun: $W_{1,2}=300 \mu \mathrm{m}, R_{s 1}, R_{D 1}, R_{D 2}=200 \Omega$. Circuit parameters of source follower : $W=300 \mu \mathrm{m}$. $R_{s}=1 \mathrm{k} \Omega$. Length of all transistors is $0.13 \mu \mathrm{m}$.

was used to probe the I and Q outputs. Because the center-to-center pitch of the tips of all probes is $150 \mu \mathrm{m}$, the dimensions of all bonding pads are $100 \mu \mathrm{m} \times 100 \mu \mathrm{m}$. This leaves a $50 \mu \mathrm{m}$ space between neighboring pads. Flexible RF cables were used to connect the probes including dc probes and test equipment in order to minimize cable loss. The cable loss, including connectors and adapters, was measured and found to be approximately $0.5 \mathrm{~dB}$ at $100 \mathrm{MHz}$.

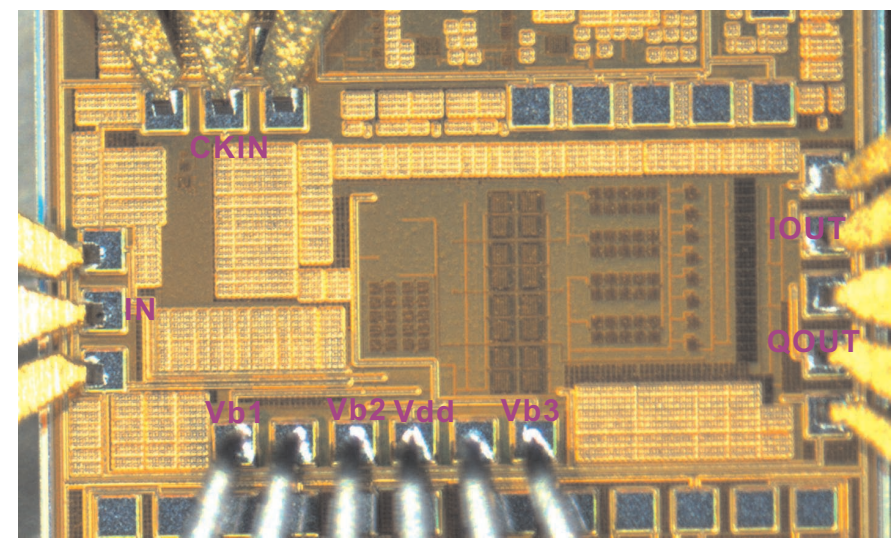

Figure 4.31: Die photo-micrograph of charge-domain sampler with embedded 4-path bandpass filter with probes in place for on-wafer measurement. 
The test set-up is shown in Fig. 4.32. Two BK precision 9124 programmable DC power supplies were used in the test set up. One for $V_{D D}$, dc biasing of the 4-path bandpass filter and common-mode voltage of charge-domain sampler, and the other one is for biasing the on-chip active balun. The ground terminals of all dc power supplies were wired to a common ground plane, in a star configuration, to avoid forming ground loops. All ground pads are connected together, on chip, to avoid any on-chip ground loops. The $100 \mathrm{MHz}$ input signal was generated using an Agilent E4420B $250 \mathrm{kHz} \sim 2.0 \mathrm{GHz}$ RF signal generator. The master clock was generated from an Agilent 81130A pattern generator. The clocks used in the 4-path bandpass filter and charge domain sampler were generated from the master clock using an on-chip clock generator. The output waveforms of the quadrature chargedomain sampler were captured using an Agilent MOS-X 3024A 200 MHz oscilloscope. The spectrum of the output of the quadrature charge-domain sampler was measured using an Agilent N9320B $9 \mathrm{kHz} \sim 3.0 \mathrm{GHz}$ spectrum analyzer.

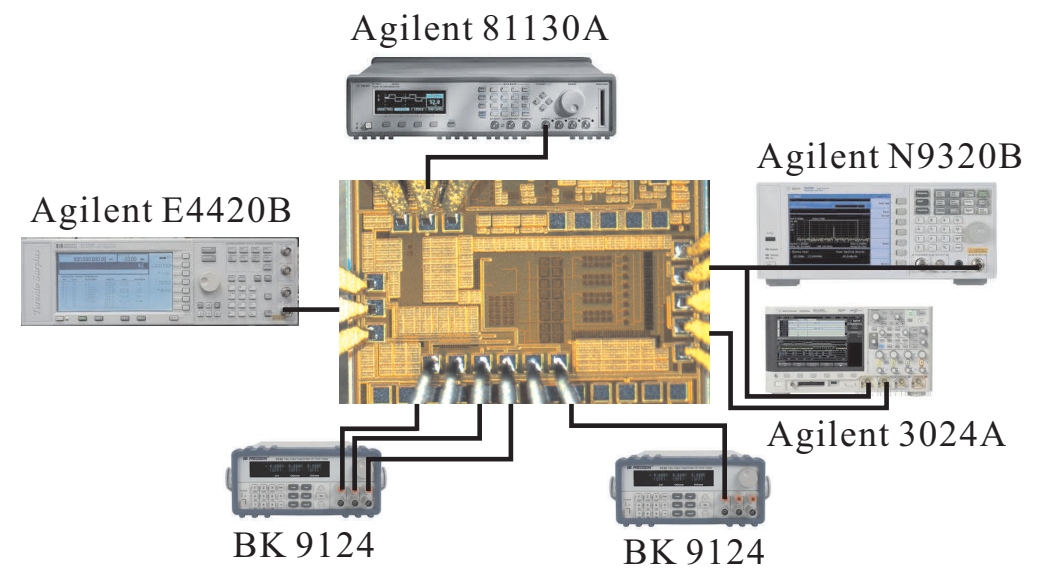

Figure 4.32: Measurement set-up.

The quadrature output signals were captured and are shown in Fig. 4.33. The measured stop-band attenuation and aliasing-band attenuation is around $40 \mathrm{~dB}$, as shown in Fig. 4.34. This was measured by sweeping the input frequency from $100 \mathrm{MHz}$ to 150 MHz. The simulated transfer function and measured transfer function are both plotted in this figure and normalized for comparison. It can be seen that, the measured frequency 
response matches simulation results closely, up to about $10 \mathrm{MHz}$. After this, the measured transfer function starts to deviate from the simulation. There are two reasons for this. The loss of the gain and the clock crosstalk. The measured gain is about $-9 \mathrm{~dB}$ rather than typical case, $+14.4 \mathrm{~dB}$ or the worst case, $+6.9 \mathrm{~dB}$ obtained in corner simulation. Given the input is $-35 \mathrm{dBm}$ and the limitation of the dynamic range of the spectrum analyzer, without noiseless pre-amplifier providing sufficient gain, it is hard to evaluate the characteristic of the filters, which are designed to provide maximum $80 \mathrm{~dB}$ attenuation for aliasing, and $60 \mathrm{~dB}$ attenuation for stop-band interferences. The reasons for the low gain is largely due to the improper on-chip biasing. The shifted operating points of the transistors break the impedance matching network. Therefore loss can be from the active balun providing input termination and the source follower output buffer that is connected to the spectrum analyzer whose input impedance is only $50 \Omega$. In addition, the degraded transconductor contributes the other part of the gain loss. The clock crosstalk also increases the difficulty of getting accurate profile of the filters. Since the limited silicon space, the improper shielding clock induces the coupled clock into the main signal path. The switching noise from the clock generator can eventually affect the $V_{T H}$ thereby changing the bias condition of the transistors. As the design is a multi-sampling system, the pattern noises located at $f_{s} / 4, f_{s} / 2 \ldots$ from gain and phase mismatch may degrade the filter performance. The large spikes were observed at the multiple of $12.5 \mathrm{MHz}$ from measurements. The measured tuning range of $25 \mathrm{MHz}$ to $250 \mathrm{MHz}$ is shown in Fig. 4.35.

For a $100 \mathrm{MHz}$ input signal, the simulated and measured stop band attenuation is $60 \mathrm{~dB}$ and $40 \mathrm{~dB}$, respectively. The simulated and measured aliasing rejection is $70 \mathrm{~dB}$ and $42 \mathrm{~dB}$, respectively. A simplified IIP3 measurement was done without using extrapolation approach. For a given sufficient small desired signal at $100.2 \mathrm{MHz}$ with $-40 \mathrm{dBm}$, the two tones, 100.4 MHz and 100.5 MHz with -30 dBm amplitude, was used to calculate IIP3. The post-layout simulation is illustrated in Fig. 4.36.

The $1 \mathrm{~dB}$ compression point is where the output of the amplifier is deviated from the ideal gain by $1 \mathrm{~dB}$. The simulated $1 \mathrm{~dB}$ compression point is $-27 \mathrm{~dB}$, shown in Fig. 4.37. 


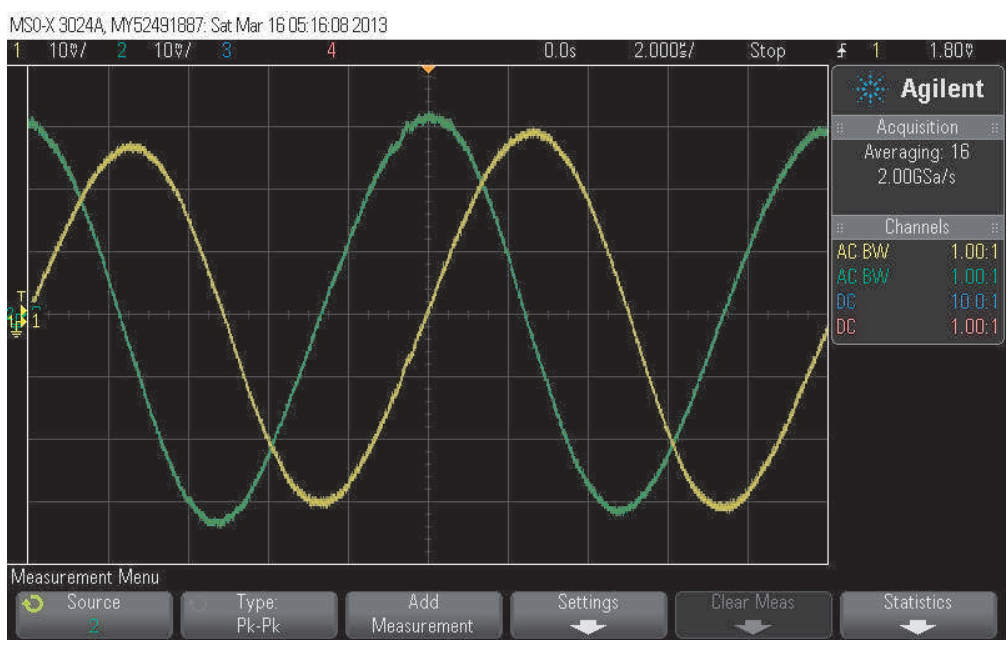

Figure 4.33: Measured quadrature output voltage of mixing sampler with input at 100.1 $\mathrm{MHz}$.

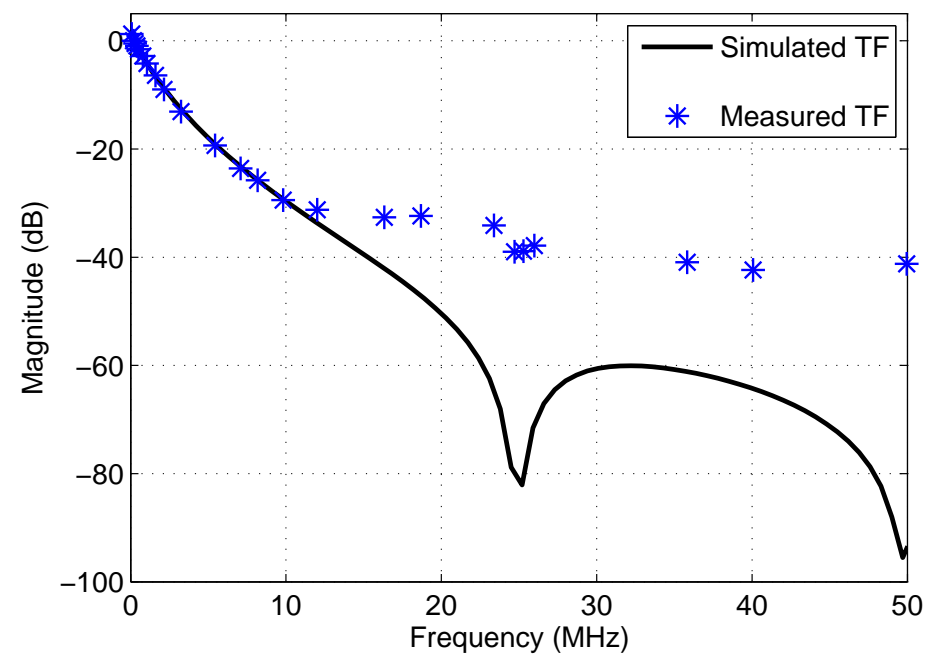

Figure 4.34: Measured and simulated normalized transfer function of mixing sampler.

From $P_{I I P 3}=\frac{\Delta P}{2}+P_{\text {fund }}$ where $\Delta P$ is the difference between input and intermodulate signal and $P_{\text {fund }}$ is the fundamental signal [2], IIP3 is obtained as $-17 \mathrm{dBm}$. The image rejection was simulated by applying the input frequencies $100 \mathrm{MHz} \pm 0.2 \mathrm{MHz}$. The 90 degree phase shift was done in Matlab. By combining the I and Q path output, the quadrature down-conversion results in an image rejection of $43 \mathrm{~dB}$. The post-layout simulation of IMR is shown in Fig. 4.38 


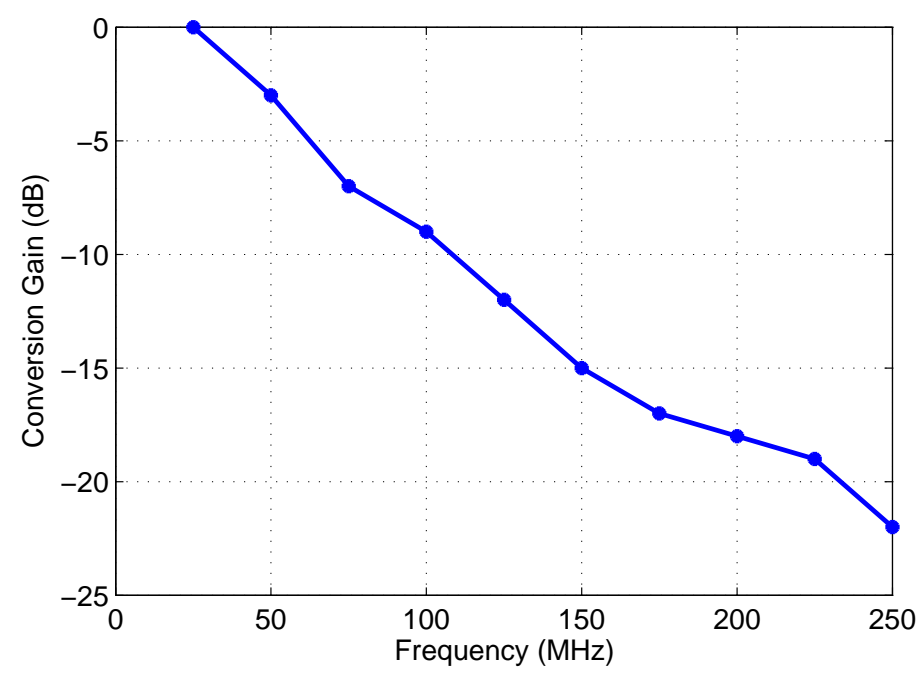

Figure 4.35: Measured tuning range of mixing sampler.

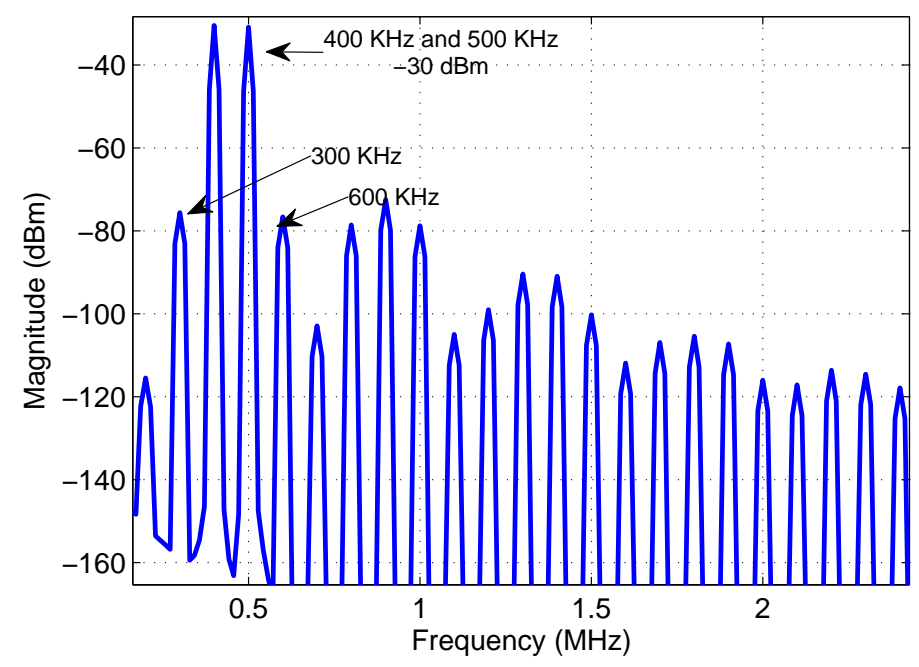

Figure 4.36: Post-layout simulated IIP3.

The chip consumes $180 \mu \mathrm{W}$ from a $1.2 \mathrm{~V}$ power supply excluding active balun and output buffer. Table 4.2 compares the performance of the design presented in this chapter to that of other published works. 


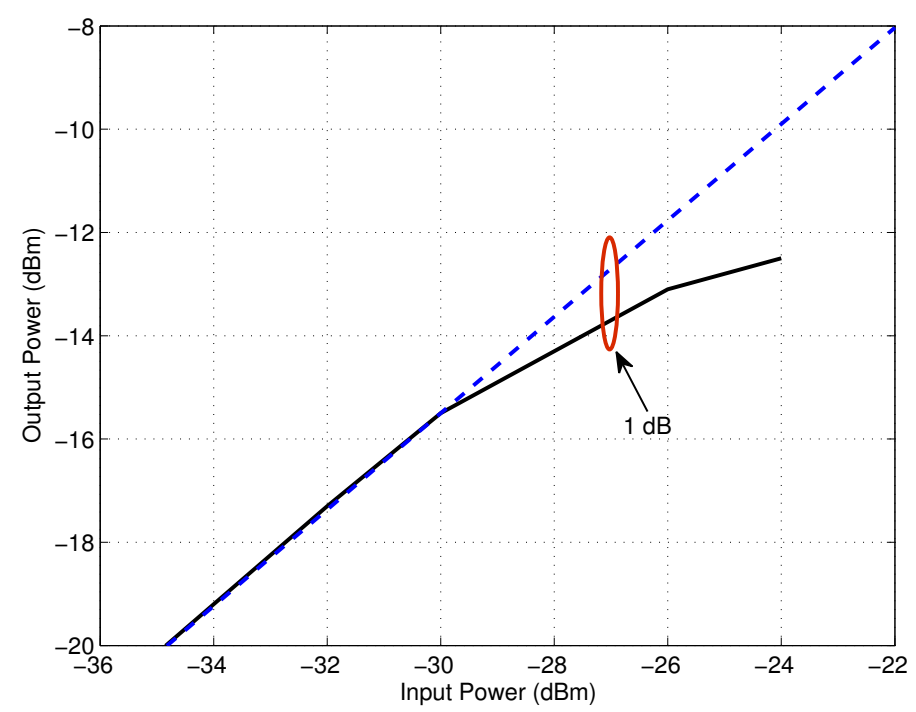

Figure 4.37: Simulated $P_{1 d B}$.

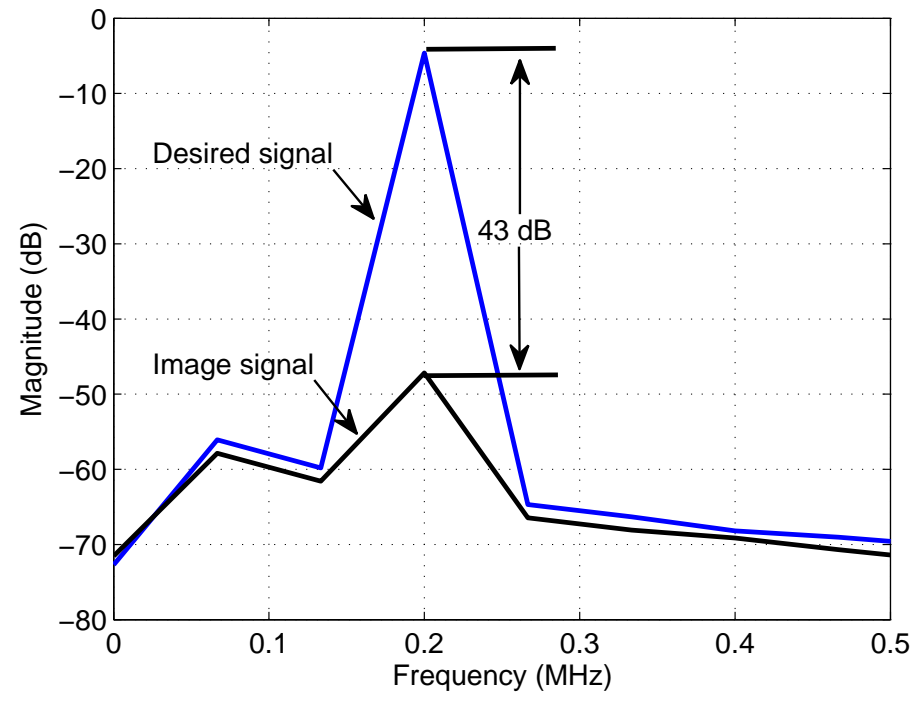

Figure 4.38: Post-layout simulated IMR.

\subsection{Summary}

The analysis and design of a low-power charge-domain quadrature down-conversion sampling mixer with embedded FIR, IIR, and 4-path bandpass filters suitable for band selection, frequency down conversion, and channel selection, are presented. Detailed inves- 
Table 4.2: Performance Summary of Sampling Mixer

\begin{tabular}{lllll}
\hline Parameters & This Work & {$[112]$} & {$[87]$} & {$[114]$} \\
\hline Technology & $0.13 \mu m$ & $0.35 \mu m$ & $90 \mathrm{~nm}$ & $90 \mathrm{~nm}$ \\
IF Frequency & $100 \mathrm{MHz}$ & $100 \mathrm{MHz}$ & Base band & $268 \mathrm{MHz}$ \\
Output Rate $f_{\text {sout }}$ & $25 \mathrm{MHz}$ & $1.85 \mathrm{MHz}$ & $30 \mathrm{MHz}$ & $120 \mathrm{MHz}$ \\
Signal Bandwidth & $800 \mathrm{KHz}$ & $923 \mathrm{KHz}$ & $250 \mathrm{MHz}$ & $20 \mathrm{MHz}$ \\
IIP3 & $-17 \mathrm{dBm}$ (simulation) & $-2 \mathrm{dBm}$ & $-22 \mathrm{dBm}$ & $0 \mathrm{dBm}$ \\
$\mathrm{P}_{1 d B}$ & $-27 \mathrm{dBm}$ (simulation) & $\mathrm{NA}$ & $-32 \mathrm{dBm}$ & $\mathrm{NA}$ \\
NF & $19.5 \mathrm{~dB}$ (simulation) & $\mathrm{NA}$ & $19 \mathrm{~dB}$ & $21.8 \mathrm{~dB}$ \\
Aliasing Rejection & $70 \mathrm{~dB}$ (simulation) & & & \\
& $40 \mathrm{~dB}$ (measurement) & $18 \mathrm{~dB}$ & $\mathrm{NA}$ & $32.5 \mathrm{~dB}$ \\
Stop Band Att. & $60 \mathrm{~dB}$ (simulation) & & & \\
& $42 \mathrm{~dB}$ (measurement) & $13 \mathrm{~dB}$ & $42 \mathrm{~dB}$ & $53 \mathrm{~dB}$ \\
\hline
\end{tabular}

tigations of the principles of periodic impulse sampling, periodic windowed sampling, and periodic N-path windowed sampling are presented. Their characteristics, with respect to the suppression of aliasing interferers and attenuation of unwanted channels, are compared. A detailed mathematical treatment of charge-domain windowed samplers with built-in sinc, FIR and IIR filters is provided. A quadrature charge-domain sampler with embedded FIR, IIR, and 4-path bandpass filters is proposed and designed. It is shown that the 4-path bandpass filter preceding the quadrature charge-domain sampling mixer provides sufficient attenuation of unwanted channels and nulls out aliasing interferers. The intrinsic sinc characteristics, FIR filtering from multi-phase integration, and IIR filtering (from the recursive operation of the history and rotating capacitors of the charge-domain sampler) provide further suppression of unwanted channels and aliasing interferers. The reused current configuration, of the 4-path bandpass filter and quadrature charge-domain sampling mixer, helps minimizing overall power consumption.

The design is applicable to zero-IF or low-IF receivers and suppresses aliasing signals, out-of-band blockers and IF image signals. Although the presented design implemented a first-order sinc and first-order FIR and IIR filters, high-order FIR filters can be implemented to provide better performance. 


\section{Chapter 5}

\section{A Cascaded Charge-domain Sampling Mixer with Embedded $\operatorname{sinc}^{3}$ FIR and N-Path Filters}

In general, a sampling mixer along with an anti-aliasing filter demands large attenuations on aliasing signal and out-of-band signal for RF receivers operating on zero-IF or low-IF topology. In Chapter 4, a charge-domain sampler embedded with CT sinc, elementary DT sinc, first-order DT IIR low-pass and band-pass filters is introduced. An evolution of previous design is implemented in this chapter. A high-order sinc FIR and 4-path band-pass filters embedded charge-domain sampling mixer is presented. The chapter is organized as following: Section 5.1 investigates the advantages of the high-order FIR filter in terms of attenuation at the notches. Section 5.2 exams the proposed sampling circuit. Simulation results are shown in the Section 5.3. A summary is provided in Section 5.4..

\subsection{Hight-Order FIR Filter}

The FIR filter which is described in Chapter 4 is to provide elementary low-pass sinc filtering function since the coefficients of the filter are all one. The first sidelob of this type filter is merely $13 \mathrm{~dB}$ attenuation. In other words, out-of-band interferences that are within the first sidelobe, are lowered by $13 \mathrm{~dB}$, which is not sufficient for most of the wireless applications. the null depth is heavily depending on the sampling frequency $f_{s}$. The sampling rate $f_{s}$ must be large enough to get the desired attenuation around $f_{s}$. On the other hand, high order filter, which use low $f_{s}$, achieve same performance. [115], [83] and 
[116] incorporated programmed coefficients of FIR filters to obtain advanced filter function in the sampler. However, the easiest approach is to utilize high-order sinc. For example. a second-order sinc in time domain is the convolution of two first-order sinc. A comparison of sinc, $\sin c^{2}$ and $\sin c^{3}$ is shown in Fig. 5.1.

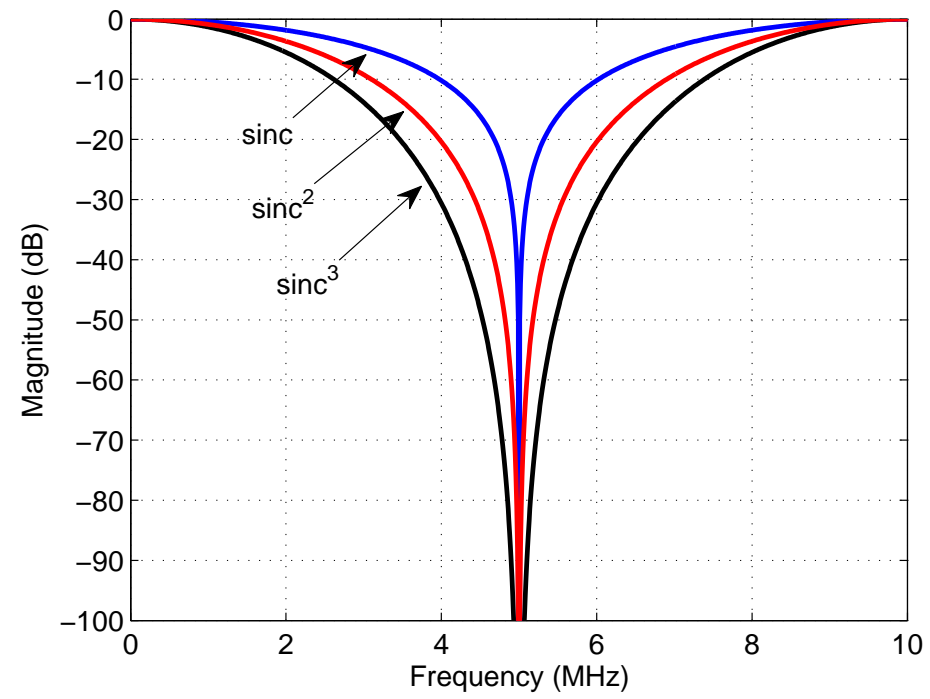

Figure 5.1: Frequency response of sinc, $\operatorname{sinc}^{2}$ and $\operatorname{sinc}^{3} . f_{s, f i r}=10 \mathrm{MHz}$, decimation ratio is 2 .

The corresponding s-domain transfer function is as follows:

$$
H(s)=\left(\frac{1-e^{-s T_{s}}}{s T_{s}}\right)^{N}
$$

where $\mathrm{N}$ is the order of the filter and $T_{s}$ is the sampling frequency. It can be seen that the higher-order of the sinc, the wider of the notch. Therefore more attenuation is added on the aliasing signals around $f_{s}$. 


\subsection{Circuit Design and Analysis}

The architecture of the charge-domain sampling mixer is shown in Fig. 5.2. Similar to the quadrature charge-domain sampler in Chapter 4, the input voltage is processed by the 4-path band-pass filter formed by M1 and a switched capacitor network that performs band selection. The band-selected output voltage obtained by diode-connected M2 is then fed to a pair of trans-conductors formed by M4 and M6 with $g_{m 6}=3 g_{m 3}$. The output currents of the trans-conductors are fed to a windowed charge-domain sampler (CDS) that functions as a low-pass filter to perform channel selection. Although a single-ended configuration is presented here, the proposed architecture is readily expendable to differential configurations to cancel out the even harmonic components and reject the common-mode signal ideally.

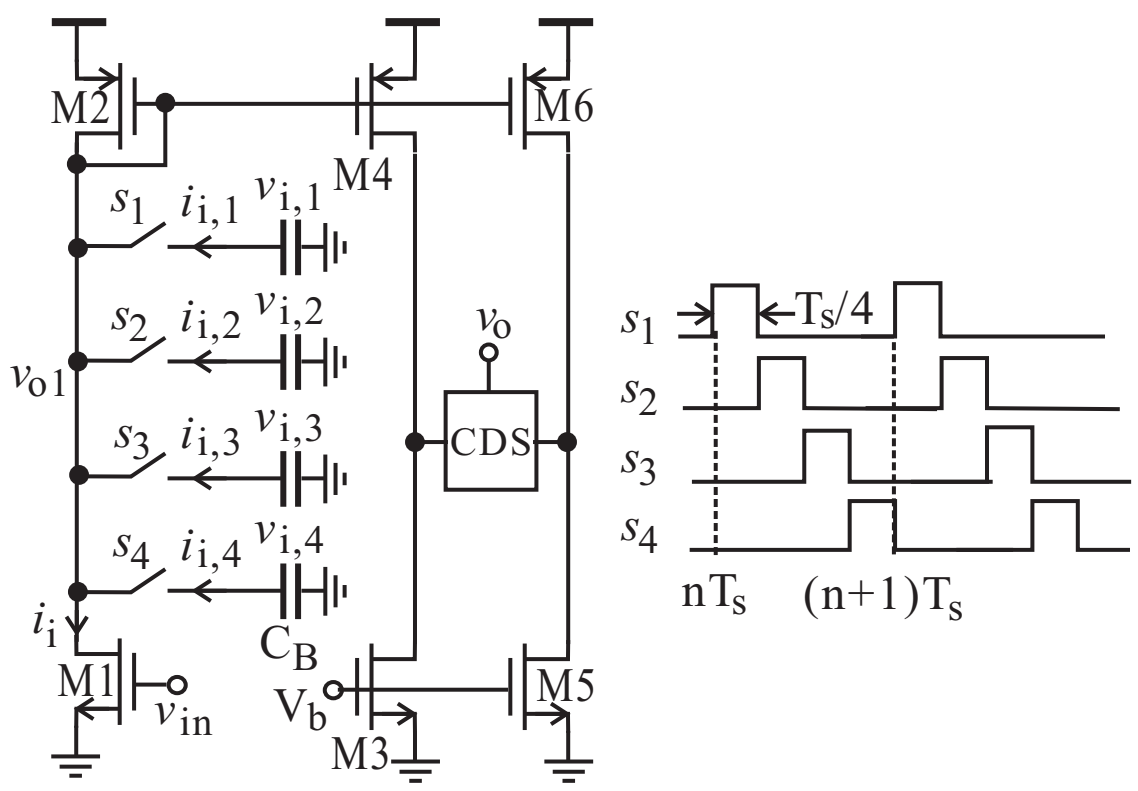

Figure 5.2: Advanced charge-domain sampler with embedded N-path band-pass filter.Circuit Parameters: $g m_{1}=2 \mathrm{mS}, g m_{4}=1.5 \mathrm{mS}, g m_{6}=4.5 \mathrm{mS}, I_{b 1}=150 \mu \mathrm{A}, I_{b 3}=235 \mu \mathrm{A}, I_{b 5}=700 \mu \mathrm{A}$, $V_{b}=335 \mathrm{mV}$. 


\subsubsection{Performance}

Fig. 5.2 shows the 4-path bandpass filter, which is the single-ended version of Fig. 4.20. $v_{i n}$ is converted to $i_{i}$ by M1. $i_{i}$ is then routed to capacitors $C_{B}$ by $s_{k}(t)$ and integrated onto $C_{B}$. The switched current $i_{i, k}(t)=i_{i}(t) s_{k}(t)$ produces voltage $v_{i, k}(t)=i_{i, k}(t) \otimes z_{B}(t)$ across the capacitors where $z_{B}(t)$ is the impulse response of the load and $\otimes$ denotes convolution. $v_{i, k}(t)$ is then sampled by $s_{k}(t)$ to produce $v_{o}(t)$. For the sake of convenience, simulated frequency response of the single-ended 4-path filter is re-plotted in Fig. 5.3. It is seen that the absence of $4^{\text {th }}$ and $8^{\text {th }}$ harmonics and the sinc-envelope are due to the frequency-shaping. It should be mentioned again that the larger the number of paths, the less attenuation on interferences located at harmonic frequencies.

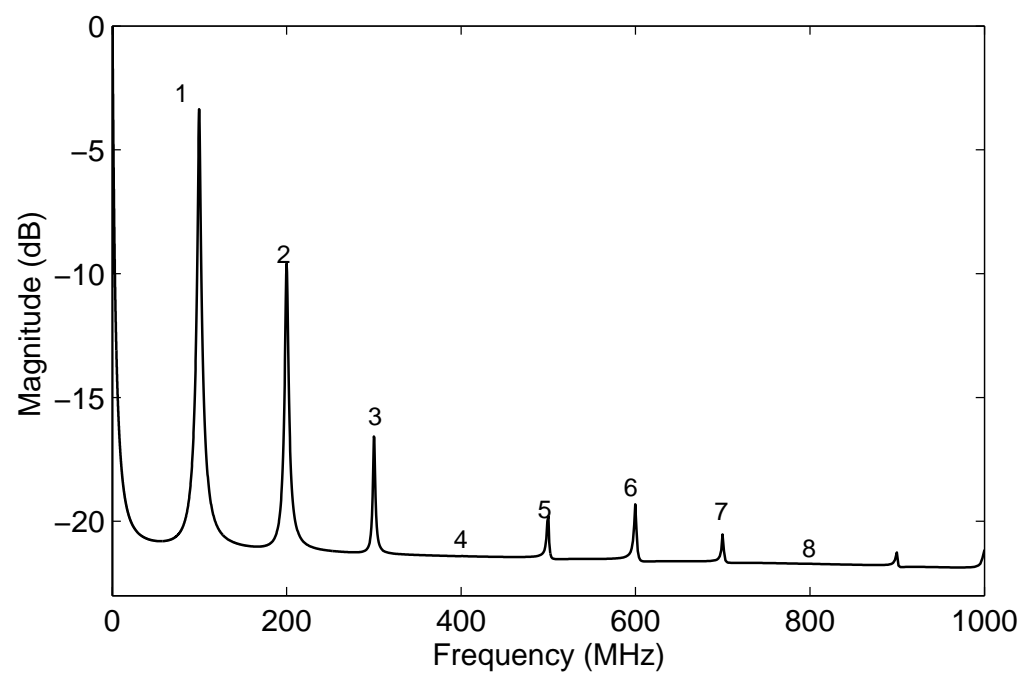

Figure 5.3: Response of 4-path band-pass filter in Fig. 5.2 ( $v_{o 1}$ excluding M3-M6 and CDS).

Fig. 5.4 shows the simplified schematic of the proposed high-order windowed chargedomain sampler. The CDS implements three distinct filters : a continuous-time (CT) sinc low-pass, a discrete-time (DT) sinc ${ }^{3}$ low-pass, and a DT sinc low-pass. The CT low-pass filtering is obtained by current integration on sampling capacitors $C_{S 1}$ with its notches determined by the integration period. To widen the nulls so as to provide a better suppression of interferences in the neighborhood of the notches, a $3^{\text {rd }}$-order sinc with the coefficients(1 
33 1) that are the results of the convolution of $\left(\begin{array}{lll}1 & 1\end{array}\right),\left(\begin{array}{ll}1 & 1\end{array}\right)$ and $\left(\begin{array}{ll}1 & 1\end{array}\right)$ is implemented by weighted input currents. The simple coefficients limit the decimation ratio to 2 to avoid aliasing, which is achieved by time-interleaved operation Fig. 5.4(b). The second stage performs another decimation of 2 . As a result, the sampling frequency is decreased by 4 times. Using a similar approach described in Chapter 4, we obtain the transfer function of the CT low-pass filter and DT filter

$$
H(j \omega)=\frac{G_{m} T_{i}}{C_{S 1}} \operatorname{sinc}\left(\frac{\omega T_{i}}{2}\right) \sum_{k=0}^{N-1} h_{k 1} z^{-k} \sum_{k=0}^{N-1} h_{k 2} z^{-k}=H_{s i n c} H_{F I R 1} H_{F I R 2}
$$

where $T_{i}$ is the pulse width of $a_{i}$ or $b_{i}, i=1,2,3, h_{k 1}$ and $h_{k 2}$ are $\left(\begin{array}{llll}1 & 3 & 3 & 1\end{array}\right)$ and $\left(\begin{array}{ll}1 & 1\end{array}\right)$ respectively.
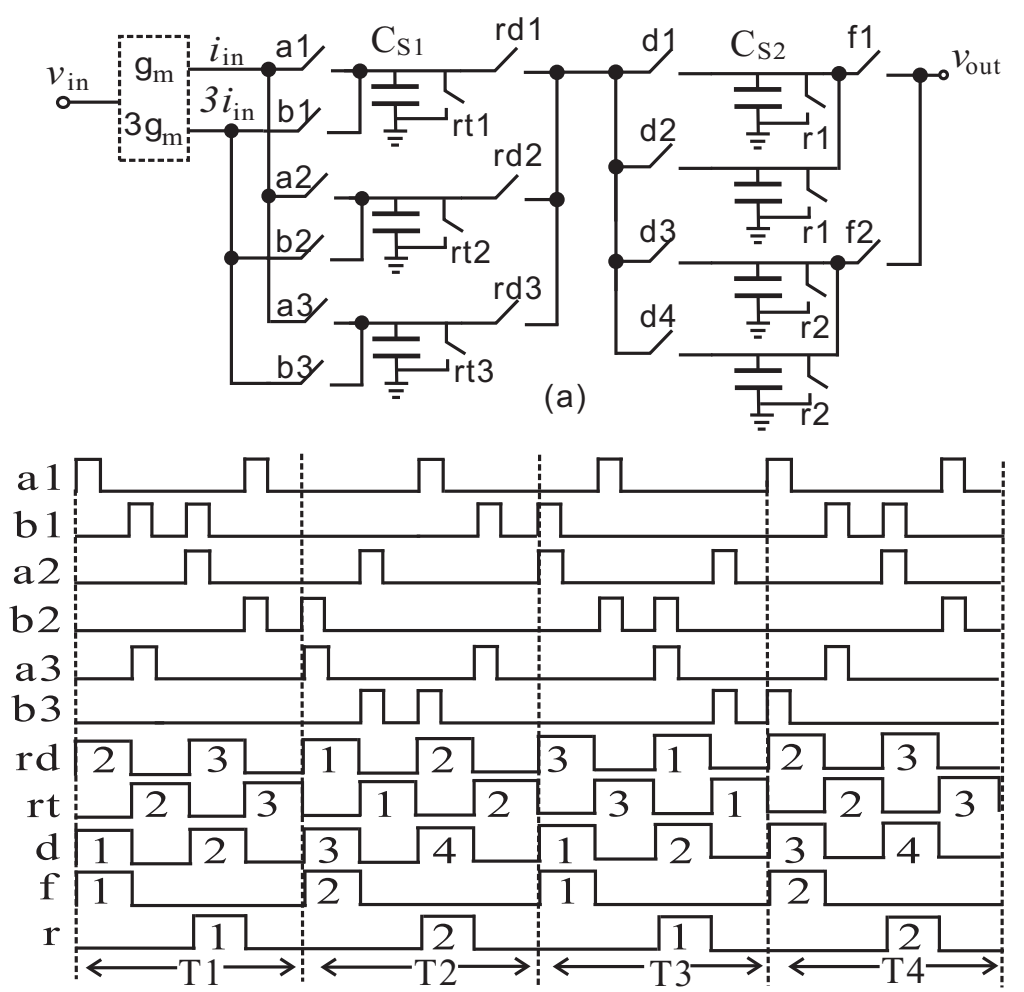

(b)

Figure 5.4: High-order windowed charge-domain sampler. Circuit Parameters: $g_{m}=1.5 \mathrm{mS}$, $C_{S 1}=10 \mathrm{pF}, C_{S 2}=1 \mathrm{pF}$. 
It is observed that the entire circuit provides the joint effect of band-pass and highorder sinc low-pass. Not only can a steep stop-band attenuation be obtained, the depth of notches will also be increased, yielding a better suppression of aliasing interferences and those in the vicinity of the notches.

\subsection{Simulation Results}

The proposed sampling mixer was designed in an IBM $130 \mathrm{~nm} 1.2 \mathrm{~V}$ CMOS and analyzed using Spectre from Cadence. Fig. 5.5 shows the normalized frequency response of the sampling mixer with a zoom-in view of the first and second notches. It is seen that the suppression of aliasing interferences at the closest notch is $80 \mathrm{~dB}$ at $920 \mathrm{KHz}-3 \mathrm{~dB}$ frequency band. Moreover, the wider aliasing band rejection at the second notch caused by sinc ${ }^{3}$ filter further improves the rejection close to $90 \mathrm{~dB}$. The minimum stop-band attenuation is $57 \mathrm{~dB}$. The gain is $9.2 \mathrm{~dB}$ compared to the calculated value of $12 \mathrm{~dB}$. The loss of the gain is mainly due to the finite output impedance of the transconductors and the non-zero ON-resistance of the switches. Fig. 5.6 shows the time response of the sampler with the $100.2 \mathrm{MHz} \mathrm{IF}$ input.

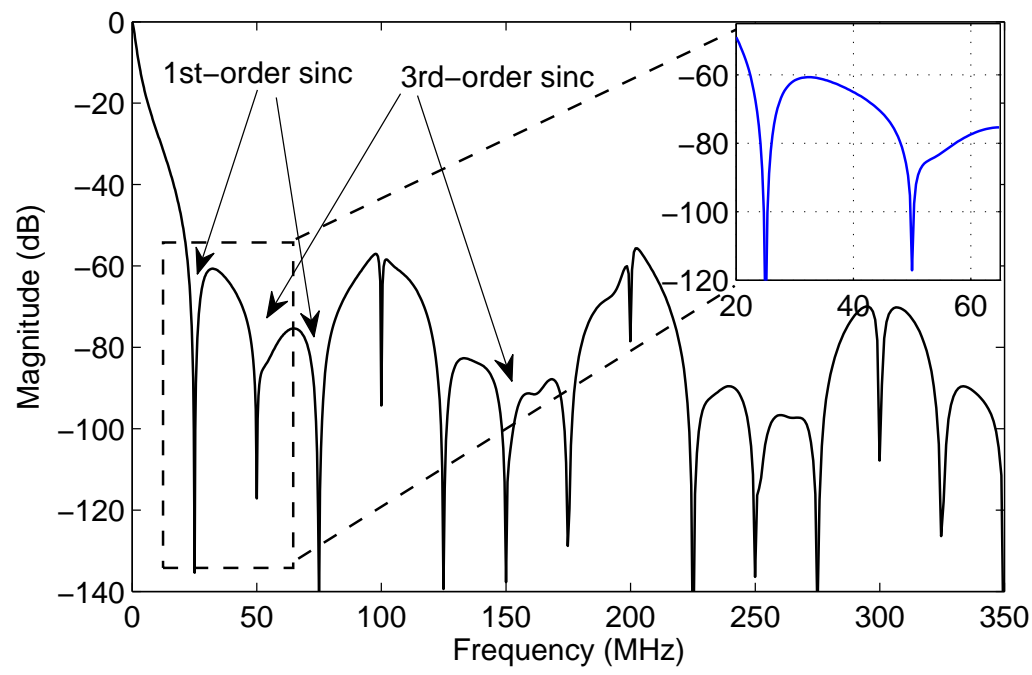

Figure 5.5: Simulated gain-normalized transfer function of proposed charge-domain windowed sampler with $100 \mathrm{MHz}$ input. 


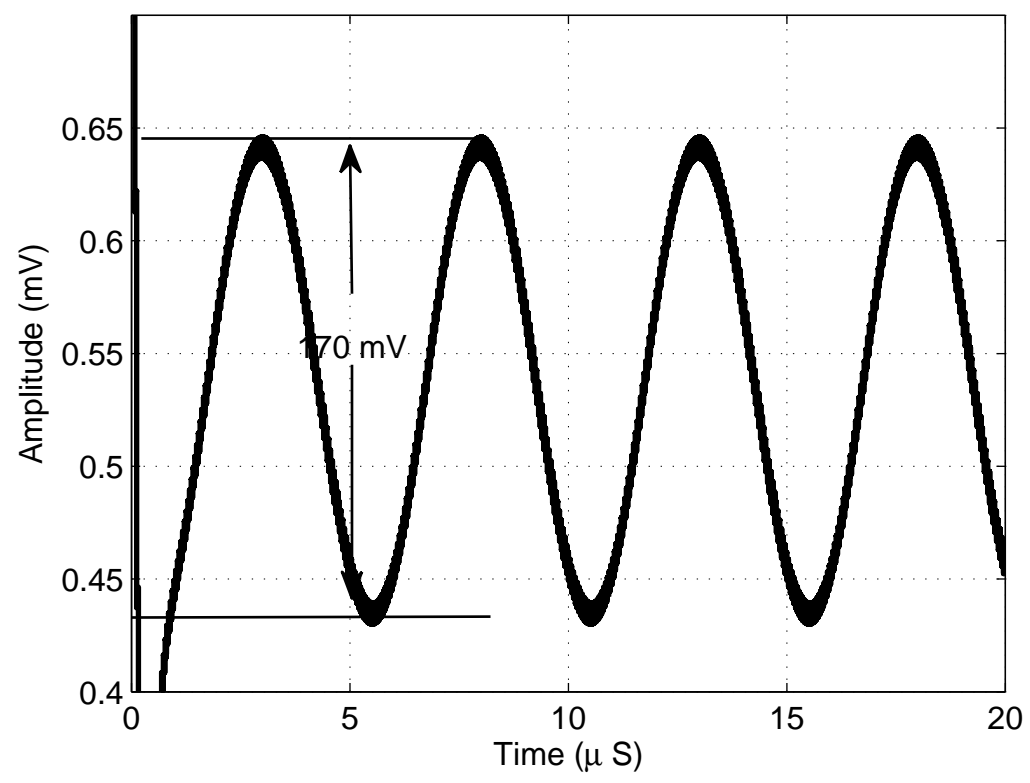

Figure 5.6: Simulated time response of the output signal with $V_{\text {in }}=50 \mathrm{mV}, f_{\text {in }}=100.2 \mathrm{MHz}$, $V_{\text {out }}=170 \mathrm{mV}, f_{\text {out }}=200 \mathrm{KHz}, f_{\text {s,out }}=25 \mathrm{MHz}$.

Fig. 5.7 shows the layout of the circuit. The entire chip is $1 \times 2 \mathrm{~mm}^{2}$. The output of the sampling mixer is fed to a common-source buffer in order to drive an off-chip RF spectrum analyzer with a $50 \Omega$ input impedance. Fig. 5.8 is the post simulation result. It can be observed that the amplitude of the output signal from post-layout simulation is smaller than that of the schematic simulation. It is mainly due to the voltage drop through the signal path in the layout.

The chip consumes $1.3 \mathrm{~mW}$ from a $1.2 \mathrm{~V}$ power supply excluding output buffer. Table 5.1 summarizes the performance of the proposed sampler and compares with that of some reported designs.

\subsection{Summary}

A charge-domain down-conversion sampling mixer with an embedded 4-path bandpass filter for band selection, a sinc ${ }^{3}$ FIR and a $1^{\text {st }}$-order sinc FIR with decimation of 4 for channel selection is presented. The sinc ${ }^{3}$ low-pass is obtained via signal weighting and time- 


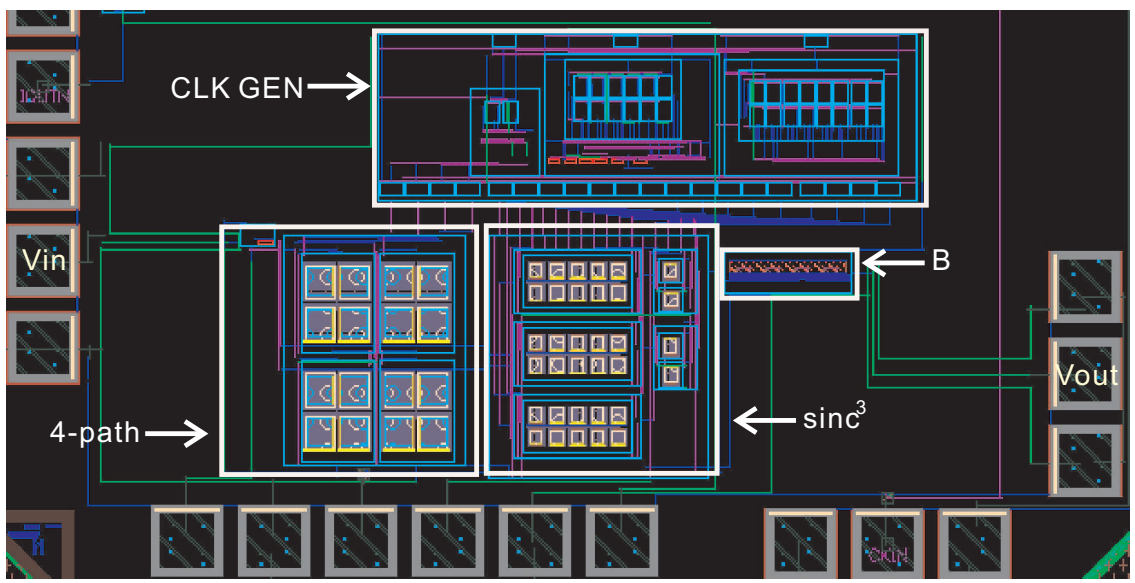

Figure 5.7: Simulated time response of the output signal with $V_{i n}=50 \mathrm{mV}, f_{\text {in }}=100.2 \mathrm{MHz}$, $V_{\text {out }}=170 \mathrm{mV}, f_{\text {out }}=200 \mathrm{KHz}, f_{\text {s,out }}=25 \mathrm{MHz}$.

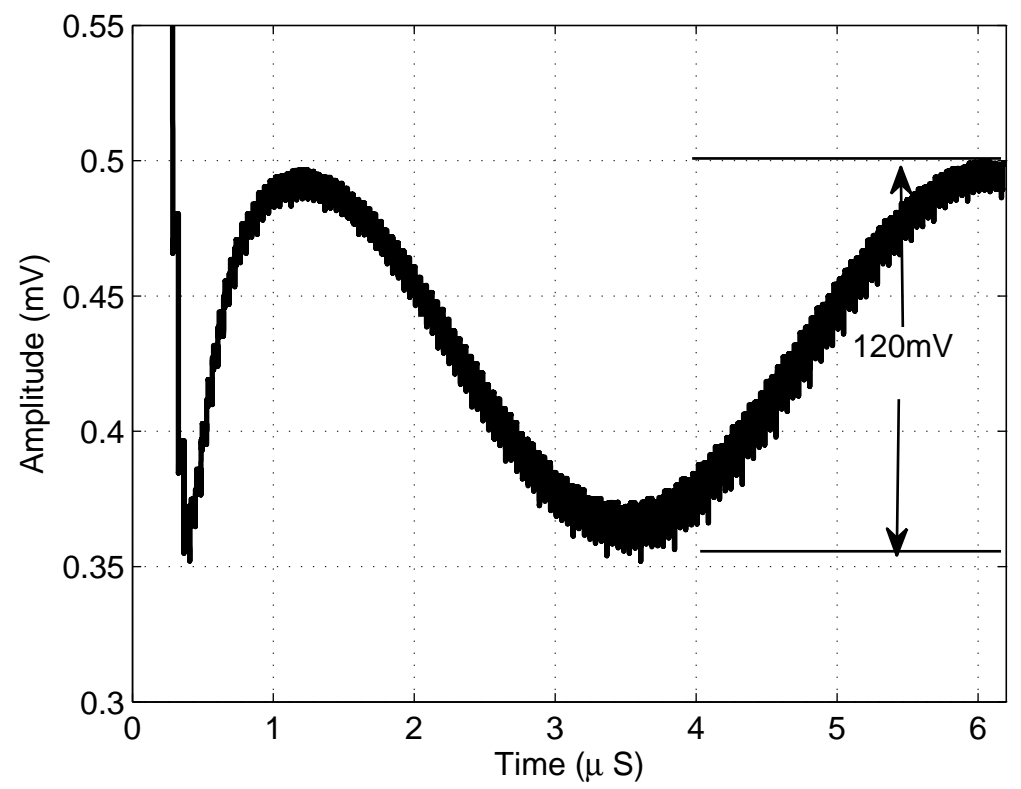

Figure 5.8: Post-simulated time response of the output signal with $V_{\text {in }}=40 \mathrm{mV}, f_{\text {in }}=100.2$ $\mathrm{MHz}, V_{\text {out }}=140 \mathrm{mV}, f_{\text {out }}=200 \mathrm{KHz}, f_{\text {s,out }}=25 \mathrm{MHz}$.

interleaving operation. It is shown that the larger attenuation on the aliasing frequency and stop-band frequency is obtained by the combination of multiple filtering functions. The sampler performs decimation by 2 with wider notches and a steeper side-lob attenuation. The total decimation by 4 is obtained by an another sinc filter following sinc ${ }^{3}$ stage. The 
Table 5.1: Performance Comparison

\begin{tabular}{llll}
\hline & This Work & {$[112]$} & {$[116]$} \\
\hline Tech. & $0.13 \mu m$ & $0.35 \mu m$ & $90 \mathrm{~nm}$ \\
Input Freq. (MHz) & 100 & 50 & 200 \\
Bandwidth (KHz) & 920 & 12900 & 850 \\
Output Sampling Freq. (MHz) & 25 & 25 & 100 \\
P1dB (dBm) & -16 & NA & NA \\
IIP3 (dBm) & -5 & NA & -25 \\
Aliasing Reject (dB) & -80 & -55 & -50 \\
Stop Band Att. (dB) & -57 & -35 & NA \\
\hline
\end{tabular}

sampling mixer designed in an IBM $130 \mathrm{~nm}$ 1.2V CMOS with $100 \mathrm{MHz}$ input exhibits 80 $\mathrm{dB}$ suppression of aliasing interferences and $57 \mathrm{~dB}$ stop band attenuation. 


\section{Chapter 6}

\section{Conclusions and Future Work}

\subsection{Conclusions}

A new theoretical approach, clearly elaborating the phenomena of injection lock for harmonic oscillators, is presented. By applying it to analyze injection-lock process, the expression of lock range of the injection-locked harmonic oscillators is derived and validated in Cadence SpectreRF based on IBM $130 \mathrm{~nm}$ technology. In addition, the work that explores the intrinsic relation between large impedance variations of active inductors and the lock range of injection-locked active inductor oscillators is completed. The theoretical findings and the demonstrated example result in good understanding of improving the lock range of injection-locked oscillators in terms of design parameters [117, 118, 119].

The multiple multi-tone injection-locked oscillators are also explored. The in-depth investigation of injection-locked non-harmonic oscillators is carried out by utilizing an approach similar to Volterra series. The larger lock range of non-harmonic oscillators with a multi-tone injection can be expected as compared with that with a single-tone injection. For the case of multiple injections, the lock range is studied analytically. And also the

proper phase assigned for each of the multiple injections is given. The theoretical analysis is validated using simulation $[120,121,122,123]$.

Two novel receivers for the application of software defined radio (SDR) are presented. The digital signal processing is performed in the analog form, resulting in reconfigurable RF 
analog front-end. The receivers resolve the issue of using discrete components, e.g. SAW filter, which is commonly employed in the conventional radio frequency receivers to select the desired frequency band. The centre frequency of this filter in designs is controlled by on-chip clocks. In comparison with the traditional design, the needed anti-aliasing filter before analog-to-digital converter is integrated in the receiver and the cut-off frequency of the filter can also be tuned by changing the sampling clocks. As a result, the entire receiver becomes fully digital controlled. The quadrature sampler has the combination of CT sinc, DT sinc, DT IIR and 4-path bandpass filter. The other one has CT sinc, DT sinc ${ }^{3}$, DT sinc and 4-path bandpass filter included.The expecting functions of the fabricated chip have been validated on the wafer station in the lab $[124,125,126]$. Although the chips are targeting IF frequency, the conceptional design can be applied to the high frequency range.

\subsection{Future Work}

With regard to injection-locked oscillators, the extension could be further increasing the lock range of the relaxation oscillators through feedback signal, representing the frequency offset from the injection signal, to adjust the tail current of the relaxation oscillators. Since the sensitivity of the frequency of the relaxation oscillator is primarily determined by the charging and discharging current, e.g. $f=I_{\text {tail }} / 2\left(C V_{\text {ref }}\right)$, given the fixed capacitor value and threshold voltage of the comparator, changing current gives rise to frequency variation. The only issue is how to detect the injection signal to determine whether it is high-injection or low-injection as they give the same frequency difference. If this difficulty can be resolved, much wider lock range can be expected. In addition, implementing the exemplified circuits on chip and testing them in the lab could be the future work as well. Extensive simulations for the theoretical analysis have been carried out. However, the measurement results will provide more solid support for the findings. Also, the example is originally intended for lowpower passive wireless communications. The relaxation oscillator is designed in subthreshold region, resulting in relatively low frequency, $13 \mathrm{MHz}$. Since the analysis is not limited to low frequency, a regular oscillator with RF band frequency can be developed to further prove the study in this thesis. The other important performance of injection-locked oscillators, 
which is not discussed, is the lock time. For multiple injections, the impacts of the phases difference between the injection signals and the oscillating signals should be clearly observed from simulations. The multiple injections leads to faster lock time in comparison with the single injection can be expected. However, these are only surmise, which need to be further investigated.

There are also more possible extensions for the programmable receivers. Future design could be focusing on the linearity and noise performance of the transconductor, which dominants the linearity and noise for the entire design. Multiple gates, source degeneration and feedback configuration can be used to improve linearity. Furthermore, one more direction is to incorporate $\Sigma \Delta \mathrm{ADC}$, which offers exceptional performance for high resolution applications. For the 4-path, in stead of bandpass filtering function, itself is a quadrature mixer. In other words, the output of DAC in the $\Sigma \Delta \mathrm{ADC}$ can be applied to 4-path network to translate baseband signal back to RF. The whole loop includes bandpass filter, quadrature mixer for feedback signal, and charge domain sampling mixer with anti-aliasing and channel selection filter. The highly integrated and better linearity can be expected. In addition, the on-wafer measurement is not able to show the entire filtering functions due to some faulty layouts for some critical signal paths, e.g. biasing voltage, receiving signal paths and etc. Since the multiple signal paths are adopted, the matching for each of path becomes essential. The leakage clock signal is observed in the post-layout simulation because of the mismatches of signal paths and improper shielding. It is worth paying close attention on the layout techniques to get better performance. 


\section{Bibliography}

[1] A. Goldsmith, Wireless communications. Cambridge: Cambridge University Press, 2005.

[2] B. Razavi, RF microelectronics. New York: Pearson education, 2012.

[3] R. Miller, "Fractional-frequency generators utilizing regenerative modulation," Proc. IRE, pp. 446-457, Jul. 1939.

[4] R. Harrison, "Theory of regenerative frequency dividers using double-balanced mixers," Proc. IEEE Int'l Microwave Symp., pp. 459-462, 1989.

[5] — - "A broad-band frequency divider using microwave varactors," IEEE Trans. Microwave Theory Tech., vol. 25, no. 12, pp. 1055-1059, Dec. 1977.

[6] H. Rategh and T. H. Lee, "Superharmonic injection-locked frequency dividers," IEEE J. Solid-State Circuits, vol. 34, no. 6, pp. 813-821, Jun. 1999.

[7] H. Rategh, H. Samavati, and T. H. Lee, "A CMOS frequency synthesizer with an injection-locked frequency divider for a $5 \mathrm{GHz}$ wireless LAN receiver," IEEE J. SolidState Circuits, vol. 35, no. 5, pp. 780-787, May. 2000.

[8] A. Mazzanti, P. Uggetti, and F. Svelto, "Analysis and design of injection-locked LC dividers for quadrature generation," IEEE J. Solid-State Circuits, vol. 39, no. 9, pp. 1425-1433, Sept. 2004.

[9] C. Cao and Y. Ding, "A 50-GHz phase-locked loop in 0.13- $\mu$ m CMOS," IEEE J. Solid-State Circuits, vol. 42, no. 8, pp. 1649-1656, Aug. 2007.

[10] H. Wu and L. Zhang, "A 16 to $18 \mathrm{GHz} 0.18 \mu \mathrm{m}$ EPI-CMOS divid-by-3 injectionlocked frequency divider," IEEE Int'l Solid-State Circuits Conf., Digest Tech. Papers, pp. 602-603, 2006.

[11] C. Wang, C. Chen, M. Lei, M. Chuang, and H. Wang, "A 66-72 GHz divide-by-3 injection-locked frequency divider in $0.13 \mu \mathrm{m}$ CMOS technology," Proc. IEEE Asian Solid-State Circuits Conference, pp. 344-347, Nov. 2007.

[12] C. L. S. Jang and W. Yeh, "A divide-by-3 injection locked frequency divider with single-ended input," IEEE Microwave and Wireless Lett., vol. 18, no. 2, pp. 142-144, Feb. 2008. 
[13] C. Zhou, L. Zhang, L. Zhang, Y. Wang, and Q. H. Z. Yu, "A $50 \%$ duty cycle widelocking range divide-by-3 divider up to $6 \mathrm{GHz}$," Proc. IEEE Radio Frequency Integrated Circuits Symp., pp. 1-4, 2011.

[14] I. T. Lee, C. H. Wang, and S. I. Liu, "Current-reused divide-by-3 injection-locked frequency divider in $65 \mathrm{~nm}$ cmos," IET Electronics Letters, vol. 47, no. 18, pp. 10291030, Sept. 2011.

[15] I. Lee, C. Wang, J. Sha, Y. Juang, and S. Liu, "D-band divide-by-3 injection-locked frequency divider in $65 \mathrm{~nm}$ CMOS," IET Electronics Letters, vol. 48, no. 17, pp. 10411042, Aug. 2012.

[16] Z. Huang, C. Wu, and B. Huang, "Design of 24-GHz 0.8-V 1.51-mW coupling currentmode injection-locked frequency divider with wide locking range," IEEE Trans. Microwave Theory Tech., vol. 57, no. 8, pp. 1948-1958, Aug. 2009.

[17] A. Mirzaei, M. Heidari, R. Bagheriand, S. Chehrai, and A. Abidi, "The quadrature LC oscillator: A complete portrait based on injection locking," IEEE J. Solid-State Circuits, vol. 42, no. 9, pp. 1916-1932, Sept. 2007.

[18] J.Lee and H. Wang, "Study of subharmonically injection-locked PLLs," IEEE J. SolidState Circuits, vol. 44, no. 5, pp. 1539-1553, May. 2009.

[19] A. Musa, R. Murakami, T. Sato, W. Chaivipas, K. Okada, and A. Matsuzawa, "A low phase noise quadrature injection locked frequency synthesizer for mm-wave applications," IEEE J. Solid-State Circuits, vol. 46, no. 11, pp. 2635-2649, Nov. 2011.

[20] J. Lu, N. Wang, and M. Chang, "A compact and low power 5-10 GHz quadrature local oscillator for cognitive radio applications," IEEE J. Solid-State Circuits, vol. 47, no. 5, pp. 1131-1140, May 2012.

[21] G. Mangraviti, B. Parvais, V. Vidojkovic, K. Vaesen, V. Szortyka, K. Khalaf, C. Soens, G. Vandersteen, and P. Wambacq, "A 52-66 GHz subharmonically injection-locked quadrature oscillator with $10 \mathrm{GHz}$ locking range in 40nm LP CMOS," Proc. IEEE Radio Frequency Integrated Circuits Symp. (RFIC), pp. 309-312, Jun. 2012.

[22] T. Siriburanon, T. Ueno, K. Kimura, S. Kondo, D. Wei, K. Okada, and A. Matsuzawa, "A 60-GHz sub-sampling frequency synthesizer using sub-harmonic injection-locked quadrature oscillators," Proc. IEEE Radio Frequency Integrated Circuits Symposium, pp. 105-108, Jun. 2014.

[23] F. Mahony, C. Yue, M. Horowitz, and S. Wong, "A 10-GHz global clock distribution using coupled standing-wave oscillators," IEEE J. Solid-State Circuits, vol. 38, no. 11, pp. 1813-1820, Nov. 2003.

[24] L. Zhang, B. Ciftcioglu, M. Huang, and H. Wu, "Injection-locked clocking: A new GHz clock distribution scheme," pp. 785-788, Sept. 2006. 
[25] L. Z. andA. Carpenter, B. Ciftcioglu, A. Garg, M. Huang, and H. Wu, "Injectionlocked clocking: A low-power clock distribution scheme for high-performance microprocessors," IEEE Trans. Very Large Scale Integration (VLSI) Systems, vol. 16, no. 9, pp. 1251-1256, Sept. 2008.

[26] L. Zhang, B. Ciftcioglu, and H. Wu, "Active deskew in injection-locked clocking," in proc. IEEE Custom Integrated Circuits Conf., Sept. 2008, pp. 567-570.

[27] M. Hossain and A. Carusone, "CMOS oscillators for clock distribution and injectionlocked deskew," IEEE J. Solid-State Circuits, vol. 44, no. 8, pp. 2138-2153, Aug. 2009.

[28] Z. Bai, X. Zhou, R. Mason, and G. Allan, "Low phase noise clock distribution network using rotary traveling wave oscillators and built-in self-test phase tuning technique," IEEE Trans. Circuits and Systems II: Express Briefs(early access), vol. PP, no. 99, pp. $1-1$, Oct. 2014.

[29] "EPC radio-frequency identity protocols class-1 generation-2 UHF RFID protocol for communications at $860 \mathrm{MHz}-960 \mathrm{MHz}$, version 1.0.9," EPCglobal Inc., 2005.

[30] C. Chan, K. Pun, K. Leung, J. Guo, L. Lincoln, and C. Choy, "A low-power continuously-calibrated clock recovery circuit for UHF RFID IC class-1 generation2 transponders," IEEE J. Solid-State Circuits, vol. 45, no. 3, pp. 587-599, March 2010.

[31] F. Marraccini, G. D. Vita, S. Pascoli, and G. Iannaccone, "Low-voltage nanopower clock generator for RFID applications," Microelectronics J., vol. 39, pp. 1736-1739, 2008.

[32] H. Lee and S. Mohammadi, "A subthreshold low phase noise cmos lc vco for ultra low power applications," IEEE Microwave and Wireless Components Letters, vol. 17, no. 11, pp. 796-798, Nov. 2007.

[33] N. Tran, B. Lee, and J. Lee, "Development of long-range UHF-band RFID tag chip using Schottky diodes in standard CMOS technology," Proc. IEEE Radio Frequency Integrated Circuits Symp., pp. 281-284, Sept. 2007.

[34] B. Fan, Y. Dai, X. Zhang, and Y. Lu, "Low power clock recovery circuit for passive HF RFID tag," Analog Integrated Circuits and Signal Processing, vol. 59, pp. 207-214, 2009.

[35] F. Kocer and M. Flynn, "A new transponder architecture with on-chip ADC for longrange telemetry applications," IEEE J. Solid-State Circuits, vol. 41, no. 5, pp. 11421148, May. 2006.

[36] N. Soltani and F. Yuan, "Analysis and design of non-harmonic injection-locked phaselocked loops with applications in remote frequency calibration of passive wireless transponders," IEEE Trans. Circuits and Systems-I: Redular papers, vol. 57, no. 12, pp. 2381-2393, Sept. 2010. 
[37] Q. Ma, M. Haider, and Y. Massoud, "Robust power oscillator design for inductivepower link applications," in Proc. IEEE Int'l Conf. Electronics, Circuits and Systems, 2011, pp. 109-112.

[38] A.Visweswaran, R. Staszewski, J. Long, and A. Akhnoukh, "Fine frequency tuning using injection-control in a $1.2 \mathrm{v} 65 \mathrm{~nm}$ CMOS quadrature oscillator," in Proc. IEEE Radio Frequency Integrated Ccicuits Symp., 2012, pp. 293-296.

[39] M.Haider, S. Islam, and M. Mahfouz, "Power-efficient injection-locked oscillator for biomedical telemetry applications," IET Electronics Letters, vol. 46, no. 18, pp. 12521254, Sept. 2010.

[40] H. Cho, J. Bae, and H. Yoo, "A $37.5 \mu \mathrm{w}$ body channel communication wake-up receiver with injection-locking ring oscillator for wireless body area network," IEEE Trans. Circuits Syst. I, vol. 60, no. 5, pp. 1200-1208, May 2013.

[41] J. Bae, L. Yan, and H. Yoo, "A low energy injection-locked FSK transceiver with frequency-to-amplitude conversion for body sensor applications," IEEE J. Solid-State Circuits, vol. 46, no. 4, pp. 928-937, April 2011.

[42] T. H. Lee, The design of CMOS Radio-Frequency integrated circuits. Cambridge: Cambridge University Press, 2004.

[43] M. McDonald, "A $2.5 \mathrm{GHz}$ BiCMOS image-reject front end," IEEE Int'l Solid-State Circuits Conf., Digest Tech. Papers, pp. 144-145, 1993.

[44] A. Hairapetian, "An $81 \mathrm{MHz}$ IF receiver in CMOS," Solid-State Circuits, IEEE Journal of, vol. 31, no. 12, pp. 1981-1986, Dec. 1996.

[45] L. Longo, R. Halim, B. Horng, K. Hsu, and D. Shamlou, "A cellular analog front end with a 98 db IF receiver," IEEE Int'l Solid-State Circuits Conf., Digest Tech. Papers, pp. 36-37, 1994.

[46] A. Mirzaei, H. Darabi, and D. Murphy, "A low-power process-scalable superheterodyne receiver with integrated high- Q filters," IEEE J. Solid-State Circuits, vol. 46, no. 12, pp. 2920-2932, Dec. 2011.

[47] A. Rofougaran, J. Chang, M. Rofougaran, and A. A. Abidi, "A 1 GHz CMOS RF front-end IC for a direct-conversion wireless receiver," IEEE J. Solid-State Circuits, vol. 31, no. 7, pp. 880-889, Jul. 1996.

[48] B. Razavi, "A 5.2-GHz cmos receiver with 62-db image rejection," IEEE J. Solid-State Circuits, vol. 36, no. 5, pp. 810-815, May. 2001.

[49] A. Garcia, C. Mishra, F. Bahmani, J. Silva-Martinez, and E. Sanchez-Sinencio, "An 11band $3-10 \mathrm{GHz}$ receiver in Sige BiCMOS for multiband OFDM UWB communication," IEEE J. Solid-State Circuits, vol. 42, no. 4, pp. 935-948, Apr. 2007. 
[50] A. Abidi, "Rf CMOS comes of age," IEEE J. Solid-State Circuits, vol. 39, no. 4, pp. 549-561, Apr. 2004.

[51] J. Mitola, "The software radio architecture," IEEE Communications Mag., vol. 33, no. 5, pp. 26-38, May 1995.

[52] A. Abidi, "The path to the software-defined radio receiver," IEEE J. Solid-State Circuits, vol. 42, no. 5, pp. 954 - 966, May 2007.

[53] B. Razavi, "A study of injection locking and pulling in oscillators," IEEE J. Solid-State Circuits, vol. 39, no. 9, pp. 1415-1424, Sept. 2004.

[54] S. Jang, C. Lin, C. Liu, and M. Juang, "An active-inductor injection locked frequency divider with variable division ratio," IEEE Microwave and Wireless Lett., vol. 19, no. 1, pp. 39-41, Jan. 2009.

[55] S. Jang, C. Liu, and C. Chuang, "A tail-injected divide-by-4 siGe HBT injection-locked frequency divider," IEEE Microwave and Wireless Lett., vol. 19, no. 4, pp. 236-238, Apr. 2009.

[56] C. Zhou, L. Zhang, L. Zhang, Y. Wang, and Q. H. Z. Yu, "Injection-lcoking-based power and speed optimization of CML dividers," IEEE Trans. on Circuits and Systems II - Express Briefs., vol. 58, no. 9, pp. 565-569, Sept. 2011.

[57] S. Jang, C. Chang, C. Cheng, C. Hsue, and C. Hsu, "A wide-locking range sige bicmos divide-by-3 injection locked oscillators," pp. 1-4, Apr. 2011.

[58] J. Kang, X. Yu, and J. Zhou, "Optimization of injection locked frequency divider with tunable active inductor," proc. IEEE Symp. Radio-Frequency Integration Technology (RFIT), pp. 74-76, Nov. 2012.

[59] M. Tsai and H. Wang, "A 0.3-2.5 GHz ultra-wideband mixer using commercial $0.18 \mu \mathrm{m}$ CMOS technology," IEEE Microwave and Wireless Component Lett., vol. 14, no. 11, pp. 522-524, Nov. 2004.

[60] E. Sackinger and W. Fischer, "A 3-GHz 32-dB CMOS limiting amplifier for SONET OC-48 receivers," IEEE J. Solid-State Circuits, vol. 35, no. 12, pp. 1884-1888, Dec. 2000 .

[61] R.Adler, "A study of locking phenomena in oscillators," Proc. of IEEE (reproduced from 1946 version.

[62] L. N, S. Patnaik, and R. Harjani, "Understanding the transient behavior of injection lcoked LC oscillators," Proc. IEEE Custom Integrated Circuits Conf., pp. 667-670, 2007.

[63] P. Maffezzoni, D. Amore, S. Daneshgar, and M. Kennedy, "Analysis and design of injection locked frequency dividers by means of a phase domain macromodel," IEEE Trans. Circuits and Systems-I: Redular papers, vol. 57, no. 11, pp. 2956-2966, Nov. 2010. 
[64] I. Ali, A. Banerjee, A. Mukherjee, and B. Biswas, "Study of injection locking with amplitude perturbation and its effect on pulling of oscillator," IEEE Trans. Circuits and Systems-I: Redular papers, vol. 59, no. 1, pp. 137-147, Jan. 2012.

[65] H. Chang, A. Borgioli, P. Yeh, and R. York, "Analysis of oscillators with external feedback loop for improved locking rang and noise reduction," IEEE J. Solid-State Circuits, vol. 47, no. 8, pp. 1535-1543, Aug. 1999.

[66] H. Chen, D. Chang, Y. Juang, and S. Lu, "A 30-GHz wideband low-power CMOS injection-locked frequency divider for $60-\mathrm{GHz}$ wireless-LAN," IEEE Microwave and Wireless Components Letters, vol. 18, no. 2, pp. 145-147, Feb. 2008.

[67] X. Yu, H. Cheema, R. Mahmoudi, A. van Roermund, and X. Yan, "A 3 mW $54.6 \mathrm{GHz}$ divide-by-3 injection locked frequency divider with resistive harmonic enhancement," IEEE Microwave and Wireless Components Letters, vol. 19, no. 9, pp. 575-577, Sept. 2009.

[68] H. Wu and A. Hajimiri, "A $19 \mathrm{GHz}, 0.5 \mathrm{~mW}, 0.35 \mu \mathrm{m}$ CMOS frequency divider with shunt-peaking locking range enhancement," IEEE Int'l Solid-State Circuits Conf., Digest Tech. Papers, pp. 412-413, 2001.

[69] J. Chien and L. Lu, "Analysis and design of wideband injection-locked ring oscillators with multiple-input injection," IEEE J. Solid-State Circuits, vol. 42, no. 9, pp. 19061915, Mar. 2007.

[70] K. Yamamoto and M. Fujishima, "70 GHz CMOS harmonic injection-locked divider," 2006, pp. 2472-2481.

[71] G. G. ad P. Kinget, "Time-domain model for injection locking in nonhamronic oscillators," IEEE Trans. Circuits Syst. I, vol. 55, no. 6, pp. 1648-1658, July 2008.

[72] H. Tong, S. Cheng, A. Karsilayan, and J. Martinez, "An injection-locked frequency divider with multiple highly nonlinear injection stages and large division ratios," IEEE Trans. Circuits Syst. II, vol. 54, no. 4, pp. 313-317, April 2007.

[73] P. Deng and J. Kiang, "A 5-GHz CMOS frequency synthesizer with an injecton-locked frequency divider and differential switched capacitors," IEEE Trans. Circuits Syst. I, vol. 56, no. 2, pp. 320-326, Feb. 2009.

[74] A. Mirzaei, S. Chehrazi, R. Bagheri, and A. Abidi, "Analysis of first-order anti-aliasing integration sampler," IEEE Trans. on Circuits and Systems I., vol. 55, no. 10, pp. 2994 $-3005,2008$.

[75] J. Jin, X. Yu, J. Zhou, and T. Yan, "Gigahertz range injection locked frequency dividers with band-width enhancement and supply rejection," IEE Electronics Letters, vol. 44, no. 17, pp. 999-1000, Aug. 2008.

[76] C. Bos and C. Verhoeven, "Frequency division using an injection-locked relaxation oscillator," in Proc. IEEE Int'l Symp. Circuits Syst., vol. 5, 2002, pp. 517-580. 
[77] K. Zhu, S. Islam, J. Holleman, and S. Yuan, "A low-power dual-modulus injectionlocked frequency divider for medical implants," in Proc. IEEE Radio and Wireless Symp., 2011, pp. 414-417.

[78] K. Zhu, S. Islam, M. Roksharifi, M. Hasan, and I. Mahbub, "A divide-by-3 0.4-1.4 GHz injection-locked frequency divider based on relaxation oscillator," IEEE Microwave and Wireless Components Letters, vol. 23, no. 7, pp. 368-370, July 2013.

[79] J. Yuan, "A charge sampling mixer with embedded filter function for wireless applications," Proc. ICMMT, pp. 315-318, 2000.

[80] R. Staszewski, K. Muhammad, D. Leipold, C. Hung, Y. Ho, J. Wallberg, C. Fernando, K. Maggio, R. Staszewski, T. Jung, J. Koh, S. John, I. Deng, V. Sarda, O. MoreiraTamayo, V. Mayega, R. Katz, O. Friedman, O. Eliezer, E. De-Obaldia, and P. Balsara, "All-digital TX frequency synthesizer and discrete-time receiver for Bluetooth radio in 130 nm CMOS," IEEE J. Solid-State Circuits, vol. 39, no. 12, pp. 2278 - 2291, December 2004.

[81] K. Muhammad, R. Staszewski, and D. Leipold, "Digital RF processing: toward lowcost reconfigurable radios," IEEE Communication Mag., vol. 43, no. 8, pp. 105 - 113, August 2006.

[82] S. Karvonen, T. Riley, and J. Kostamovaara, "Charge-domain FIR sampler with programmable filtering coefficients," IEEE Trans. on Circuits and Systems II., vol. 53, no. 3, pp. 192 - 196, March 2006.

[83] S. Karvonen, T. Riley, S. Kurtti, and J. Kostamovaara, "A quadrature charge-domain sampler with embedded FIR and IIR filtering functions," IEEE J. Solid-State Circuits, vol. 41, no. 2, pp. 507 - 515, Feb. 2006.

[84] R. Bagheri, A. Mirzaei, S. Chehrazi, M. Heidari, M. Lee, M. Mikhemar, W. Tang, and A. Abidi, "An $800 \mathrm{MHz}-6 \mathrm{GHz}$ software-defined wireless receiver in 90-nm CMOS," IEEE J. Solid-State Circuits, vol. 41, no. 12, pp. 2860-2876, Dec. 2006.

[85] R. Bagheri, A. Mirzaei, M. Heidari, S. Chehrazi, M. Lee, M. Mikhemar, W. Tang, and A. Abidi, "Software-defined radio receivers: dream to reality," IEEE Communications Mag., vol. 44, no. 8, pp. 111 - 118, August 2006.

[86] A. Mirzaei, S. Chehrazi, R. Bagheri, and A. Abidi, "A second-order anti-aliasing prefilter for a software-defined radio receiver," IEEE Trans. on Circuits and Systems I., vol. 56, no. 7, pp. 1513 - 1524, 2009.

[87] A. Yoshizawa and S. Iida, "A 250-MHz cutoff charge-domain baseband filter with improved stop-band attenuations," IEEE Radio Frequency Integrated Circuits Symp., Dig. Papers, pp. 491-494, 2009. 
[88] M. Darvishi, R. V. D. Zee, E. Klumperink, and B. Nauta, "A 0.3-to-1.2 GHz tunable 4th-order switched $g_{m}$-C bandpass filter with $55 \mathrm{~dB}$ ultimate rejection and out-ofband IIP3 of +29 dBm," IEEE Int'l Solid-State Circuits Conf., Dig. Tech. Papers, pp. 358-361, 2011.

[89] A. Ghaffari, E. Klumperink, M. Soer, and B. Nauta, "Tunable high-Q N-path bandpass filters: modeling and verification," IEEE J. Solid-State Circuits, vol. 46, no. 5, pp. 998-1011, May 2011.

[90] A. Mirzaei, H. Darabi, and D. Murph, "Architectural evolution of integrated M-phase high-Q bandpass filters," IEEE Trans. on Circuits and Systems I., vol. 59, no. 1, pp. 52-65, Jan. 2012.

[91] B. Razavi, Design of Analog CMOS Integrated Circuits. New York: McGraw-Hill, 2001.

[92] F. Yuan, CMOS active inductors and transformers - principle, implementation, and applications. New York: Springer, 2008.

[93] Y. Wu, X. Ding, M. Ismail, and H. Olsson, "RF bandpass filter design based on cmos active inductors," IEEE Trans. Circuits and Systems-II: Express briefs, vol. 50, no. 12, pp. 942-949, Dec. 2003.

[94] T. Lin and A. Payne, "Design of a low-voltage, low-power, wide-tuning integrated oscillator," Proc. IEEE Int'l Symp. Circuits and Systems, vol. 5, pp. 629-632, 2000.

[95] S. Hara, T. Tokumitsu, T. Tanaka, and M. Aikawa, "Lossless broad-band monolithic microwave active inductors," IEEE Trans. Microwave theory and applications, vol. 37, no. 12 , pp. 1979-1984, Dec. 1989.

[96] B. Razavi, "A study of phase noise in CMOS oscillators," IEEE J. Solid-State Circuits, vol. 31, no. 3, pp. 331-343, Mar. 1996.

[97] S. Shekhar, M. Mansuri, F. O'Mahony, G. Balamurugan, J. Jaussi, J.Kennedy, D. J. Allstot, R. Mooney, and B. Casper, "Strong injection locking in low-Q LC oscillators: Modeling and application in a forwarded-clock I/O receiver," IEEE Trans. Circuits and Systems-I: Redular papers, vol. 56, no. 8, pp. 1818-1829, Aug. 2009.

[98] S. Mitra, Digital Signal Processing : A Computer-Based Approach. Boston: McGrawHill, 2006.

[99] M. Schetzen, The Volterra and Wiener Theory of Nonlinear Systems. New York: John Wileys and Sons, 1981.

[100] P. Wampacq and W. Sansen, Distortion Analysis of Analog Integrated Circuits. Boston: Kluwer Academic Publishers, 1998.

[101] F. Yuan and A. Opal, "Distortion analysis of periodically switched nonlinear circuits using time-varying volterra series," IEEE Trans. on Circuits and Systems I - Fundamental Theory and Applications, vol. 48, no. 6, pp. 726-738, Jun. 2001. 
[102] — - "An efficient transient analysis algorithm for mildly nonlinear circuits," IEEE Trans. on Computer-Aided Design of Integrated Circuits Syst., vol. 21, no. 6, pp. 662673, Jun. 2002.

[103] Q. Li and F. Yuan, "Time domain response and sensitivity of periodically switched nonlinear circuits," IEEE Trans. on Circuits and Systems I - Fundamental Theory and Applications, vol. 50, no. 11, pp. 1436-1446, Nov. 2003.

[104] R. Thomas and A. Rosa, The Analysis and Design of Linear Circuits. New York: John Wileys and Sons, 2001.

[105] A. Foyt and T. Quist, "Phase-locked gaas cw microwave oscillators," IEEE Trans. on Electron Devices., vol. 13, pp. 662-673, Jan. 1966.

[106] H. Stover, "Theoretical explanation for the output spectra of unlocked driven oscillators," Proceedings of the IEEE, vol. 54, pp. 310-311, Feb. 1966.

[107] C. Stancampiano and S. Shapiro, "Power spectrum of an injection-locked josephon oscillator," IEEE Trans. on Magnetics., vol. MAG-11, pp. 800-803, Mar. 1975.

[108] C. Campbell, "Beat frequency spectra in a driven unlocked multimode saw cmob oscillator," Proc. Ultrasonic Symp., pp. 69-71, 1987.

[109] K. Tsai, J. Wu, and S. Liu, "Frequency dividers with enhanced locking range,," Proc. IEEE Radio Frequency Integrated Circuits Symp., pp. 661-664, 2008.

[110] Y. Wan, X. Lai, and J. Roychowdhury, "Understanding injection locking in negativeresistance lc oscillators intuitively using nonlinear feedback analysis," Proc. IEEE Custom Integrated Circuits Conf., pp. 729-732, 2005.

[111] L. Franks and I. Sandberg, "An alternative approach to the realization of network transfer functions: The N-path filters," Bell Systems Tech. J., vol. 39, pp. 1321-1350, Sept. 1960.

[112] S. Karvonen, T. Riley, and J. Kostamovarra, "A CMOS quadrature charge-domain sampling circuit with 66-dB SFDR up to $100 \mathrm{MHz}$," IEEE Trans. on Circuits and Systems I, vol. 52, no. 2, pp. 292 - 304, Feb. 2005.

[113] S. Karvonen, T. Riley, and J. Kostamovaara, "A low noise quadrature subsampling mixer," Proc. IEEE Int'l Symp. on Circuits and Systems, vol. 3, pp. 790-793, 2001.

[114] M.Huang and T. Chiu, "A quadrature charge-domain filter with freqyency downconversion for RF receivers," IEEE Trans. on Microwave Theory and Techniques, vol. 58, no. 5, pp. 1323-1332, May. 2010.

[115] D.Jakonis, K. Folkesson, J. Dabrowski, P. Eriksson, and C. Svensson, "A 2.4 GHz RF sampling receiver front-end in $0.18 \mu \mathrm{m}$ CMOS," IEEE J. Solid-State Circuits, vol. 40, no. 6, pp. 1265-1277, Jun. 2005. 
[116] N. Nguten, H. Jung, H. Min, J. Lee, G. Lee, S. Eo, and K. Yu, "A low power discretetime receiver for triple-band FM/T-DMB/DAB system-on-chip," Proc. IEEE CICC, pp. 311-314, 2011.

[117] Y. Zhou and F. Yuan, "Subthreshold CMOS active inductors with applications to low-power injection-locked oscillators for passive wireless microsystems," Proc. IEEE Mid-West Symp. Circuits Syst, pp. 885-888, Aug. 2010.

[118] — , "A comparative study of lock range of injection-locked active inductor oscillators," Proc. IEEE Mid-West Symp. Circuits Syst, pp. 793-796, Aug. 2010.

[119] — - "A study of lock range of injection-locked CMOS active-inductor oscillators using a linear control system approach," IEEE Trans. on Circuits and Systems II - Express Briefs., vol. 58, no. 10, pp. 627-631, Oct. 2011.

[120] — , "A freuqncy-domain study of lock range of harmonic oscillators with multiple injections," Proc. IEEE North-East Workshop. Circuits Syst, pp. 29-32, Apr. 2012.

[121] F. Yuan and Y. Zhou, "A phasor-domain study of lock range of harmonic oscillators with multiple injections," IEEE Trans. on Circuits and Systems II - Express Briefs., vol. 59, no. 8, pp. 466-470, Oct. 2012.

[122] — , "Frequency-domain study of lock range of non-harmonic oscillators with multiple multi-tone injections," IEEE Trans. on Circuits and Systems I - Regular Papers, vol. 60, no. 6, pp. 1395-1406, Jun. 2013.

[123] Y. Zhou and F. Yuan, "A study of injection-locked non-harmonic oscillators using Volterra series," IET Circuits Devices and Systems(accepted for publication), 2014.

[124] Y. Zhou, N. Filiol, and F. Yuan, "Low-power programmable charge-domain sampling mixer with embedded N-path bandpass filter for software-defined radio," Proc. IEEE Int'l Symp. Circuits Syst, pp. 1934-1937, May. 2013.

[125] Y. Zhou and F. Yuan, "Cascaded charge-domain sampling mixer with embedded 3rdorder sinc FIR and 4-path filters for software-defined radio," IET Electronics Letters, pp. 216-218, Jan. 2014.

[126] — - "Current reuse quadrature charge-domain sampling mixer with embedded FIR, IIR, and N-path filters," IEEE Trans. on Circuits and Systems I - Regular Papers (under revision), 2014. 